\title{
LEPTIN RECEPTOR, A SURFACE MARKER FOR A SUBSET OF HIGHLY \\ ENGRAFTING LONG-TERM FUNCTIONAL HEMATOPOIETIC STEM CELLS
}

Thao Le Phuong Trinh

Submitted to the faculty of the University Graduate School

in partial fulfillment of the requirements

for the degree

Doctor of Philosophy

in the Department of Microbiology and Immunology, Indiana University

April 2021 
Accepted by the Graduate Faculty of Indiana University, in partial fulfillment of the requirements for the degree of Doctor of Philosophy.

\title{
Doctoral Committee
}

Hal E. Broxmeyer, PhD, Chair

Edward F. Srour, PhD

January 26, 2021

\author{
Reuben Kapur, PhD
}

Utpal Dave, MD 
(C) 2021

Thao Le Phuong Trinh 


\section{ACKNOWLEDGEMENTS}

First and foremost, I would like to thank my Ph.D. mentor, Dr. Hal Broxmeyer. I still remember vividly on the first snow day of 2016 when I had my very first committee meeting as a Ph.D. candidate in Dr. Maria Grant's Lab (my former Ph.D. lab before Dr. Grant left the school). There were three members who were able to attend the meeting that day, and Hal was the only person who enthusiastically paused me on at least 3-5 times on each slide to ask questions and provided thoughtful comments. Even though I had a little difficulty understanding his New Yorker accent, he made sure that I knew what I was talking about. He took the time to explain every little detail and corrected me on every terminology I used wrongly. He was so nice that he even offered to meet later if I needed additional help. That was exactly the moment I realized the type of mentorship I was searching for. Maria was the person who introduced me to stem cell biology, and through her I got to know Hal. I am thankful for her guidance and the Grant Lab's support at the beginning of my PhD training. But it was Hal who kindly and patiently provided me endless opportunities to explore and taught me how to do science properly and rigorously.

Right at this moment, I am still somewhat in awe that I am writing my thesis. It felt like just yesterday when Hal taught me for the first time how to distinguish different mouse and human colony morphologies. During the first two years I basically had little to no data that seemed to shed "light at the end of the tunnel," 
but after every single meeting Hal always tried to make me believe in myself and never give up trying. Being your student is not only an honor. I am humble and grateful to be part of your lab. Thank you for inspiring me throughout my Ph.D. training and for the rest of my career.

I would also like to thank my committee members - Dr. Edward F. Srour, Dr. Reuben Kapur, Dr. Utpal Dave, and Dr. Raghu Mirmira (former member). Dr. Srour has spent a great amount of time to help me with flow cytometry-based techniques; he patiently went through my data analyses and guided me with my first transplant experiment. Thank you so much Dr. Srour for teaching me whenever I needed your inputs. My special thanks to Dr. Kapur and Dr. Dave for providing me so much insightful feedback during each committee meeting.

My sincere thanks to other individuals who have directly helped me to finish my Ph.D. project - Dr. James Ropa, Dr. Arafat Aljoufi, Scott Cooper, and Anthony Sinn. I would like to thank you Jim for doing an outstanding job on analyzing my RNA-seq data in a timely manner, which was a very important part of my paper. I also want to thank you Arafat, Scott and Tony - you guys have helped me tremendously with your excellent skills and professionalism. Without your help, it would have not been feasible for me to obtain all the data and complete this work. 
My experiments have not been possible without the excellent work done by all the core facility staff members. My special thanks to Andy, Garrett, Kim and Kim at the Flow Cytometry Core; I also would like to thank Melissa, Kathy and Savannah at the In Vivo Core Facility here at Indiana University. I am grateful for all your help. Every single step of an experiment is important, so your contribution is very much appreciated. Thank you for performing your job with great care and consistency.

My special thanks to the Medical Scientist Training Program (MSTP) directors Dr. Maureen Harrington, Dr. Raghu Mirmira, Dr. Wade Clapp, Dr. Britney Herbert, Dr. Benjamin Gaston and the selection committee. Thank you for giving the opportunity to be part of this wonderful program.

I would also thank the Microbiology and Immunology Department and everyone in the Broxmeyer's Lab (Dr. Maegan Capitano, Dr. Xinxin Huang, Dr. Bin Guo) and others for all the support throughout my entire Ph.D. training. I am especially thankful for the continuous guidance from Dr. Bauer and Ms. Cynthia Booth.

To all my wonderful teachers both in Vietnam and the U.S., I am grateful for the care and support you all have given me throughout the years. I want to send my special thanks to Mrs. Belinda Jons, Dr. Sean Putnam, Dr. Georgia Stevens, Dr. Erin Sayer and Dr. Janos Zempleni. Thank you for believing me and helping me 
with everything you could during one of the toughest times in my life in high school and college.

Finally, I would not ever be able to get to this point in my professional career without the endless love and sacrifice from my parents, my grandmother and my sister. I know that life has always been tough for our regardless whether we were in Vietnam or America, but you have always been there for me when I felt lost. Thank you for giving me a chance to pursue my aspiration. I know that I am the luckiest kid in this world since the day I was born, and that is because of my loving parents and my extraordinarily inspirational grandma.

And to all my crazy, fun and closest friends (Abass, Farooq, Bernard, Tim, Quynh, Oat, Teresa, Tuan) thank you for making my life more 'colorful.' 
Thao Le Phuong Trinh

\section{LEPTIN RECEPTOR, A SURFACE MARKER FOR A SUBSET OF HIGHLY ENGRAFTING LONG-TERM FUNCTIONAL HEMATOPOIETIC STEM CELLS}

The entire hematopoietic system rests upon a group of very rare cells called hematopoietic stem cells (HSCs). Due to this extraordinarily crucial role, after birth HSCs are localized to the deep bone marrow niche, a hypoxic environment inside the bone where HSCs are under well-orchestrated regulation by both cellular and humoral factors. Among the cellular components regulating hematopoiesis are Leptin Receptor (LEPR)-expressing mesenchymal/stromal cells and adipocytes; both have been demonstrated to have significant influence on the maintenance of HSCs under homeostasis and in stress-related conditions. It has been reported in early work by others that HSCs and hematopoietic progenitor cells (HPCs) express LEPR. However, whether LEPR ${ }^{+} \mathrm{HSC} / \mathrm{HPCs}$ are functionally different from other HSCs/HPCs was unknown. In this study, I demonstrated for the first time that murine LEPR ${ }^{+}$Lineage-Sca- $1^{+}{ }^{+} \mathrm{CKit}^{+}(\mathrm{LSK}, \mathrm{a}$ heterogenous population consisting of HSCs/HPCs) cells even though constituting a small portion of total LSK cells are significantly enriched for both phenotypic and functional self-renewing long-term (LT) HSCs as shown in primary and secondary transplants in lethally irradiated recipients. LEPR ${ }^{+}$LSK cells are also more enriched for colony-forming progenitor cells assessed by colony-forming unit (CFU) assays. In addition, LEPR+ HSCs (defined as LSKCD $150^{+} \mathrm{CD}^{-} 8^{-}$) exhibited robust repopulating potential as compared to LEPR- 
HSCs in long-term competitive transplantation assays. To elucidate the molecular pathways that may govern functional properties of LEPR ${ }^{+} H S C s$, bulk RNA-seq on freshly sorted cells was done. Gene set enrichment analyses (GSEA) revealed Interferon Type I and Interferon y (IFNY) Pathways were significantly enriched in LEPR ${ }^{+} H S C s$ while mitochondrial membrane protein gene set was significantly enriched in LEPR-HSCs. Interestingly, proinflammatory signaling including IFNy pathway has been suggested to be critical for the emergence of embryonic HSCs from the hemogenic endothelium. Altogether, our work demonstrated that LEPR ${ }^{+} H S C$ s represent a small subset of highly engrafting adult BM HSCs. These results may have potential therapeutic implications in the field of hematopoietic transplantation as LEPR is highly conserved between mice and humans.

Hal E. Broxmeyer, Ph.D, Chair 


\section{TABLE OF CONTENTS}

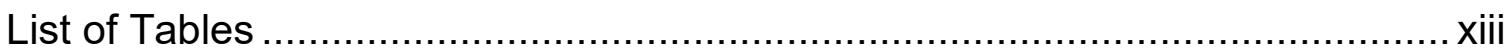

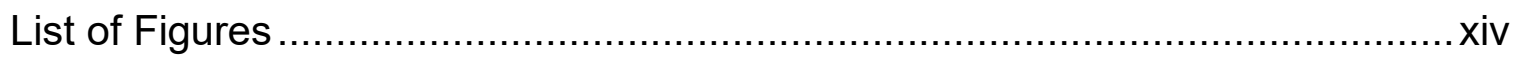

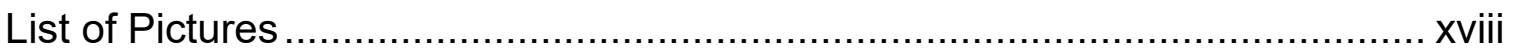

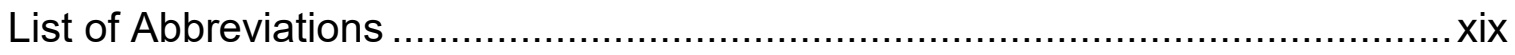

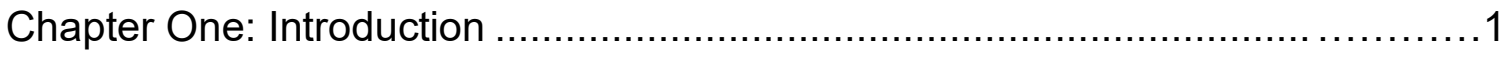

1.1 Hematopoietic cell transplantation ..................................................... 1

1.1.1 Sources of transplantable cells - advantages and disadvantages ............. 2

1.1.2 Efforts to improve clinical outcomes for patients receiving HCT ............... 4

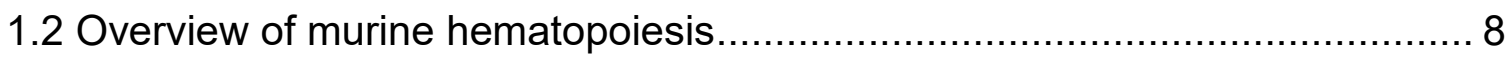

1.2.1 Emergence of HSCs during embryonic hematopoiesis .......................... 9

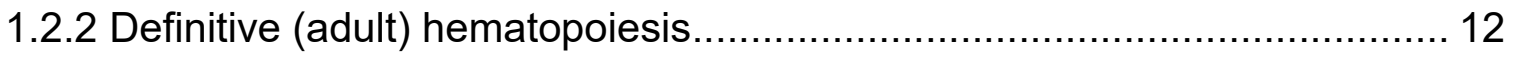

1.2.2.1 Adult hematopoietic stem and progenitor cells ................................ 12

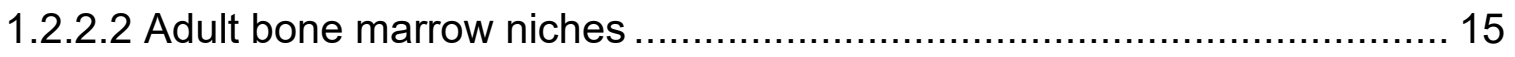

1.3 Hematopoiesis during ageing and increased risks for malignancies ........... 18

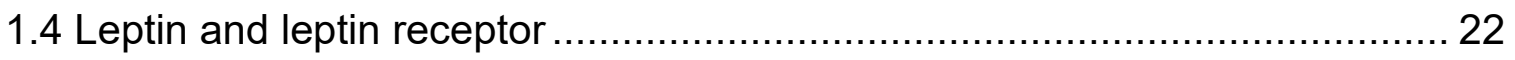

1.4.1 Discoveries of leptin/leptin receptor and their roles in non-hematopoietic

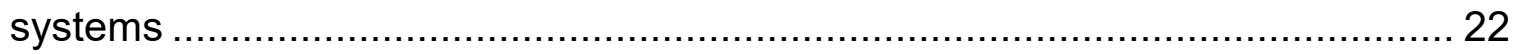

1.4.2 Leptin/leptin receptor in hematopoiesis .......................................... 24

1.4.2.1 Leptin/leptin receptor in adult non-malignant hematopoiesis, via

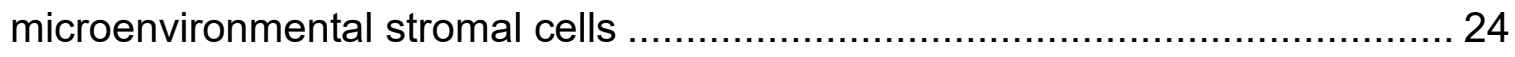

1.4.2.2 Leptin/leptin receptor in hematopoietic stem and progenitor cells ......... 27 
1.4.2.3 Leptin/leptin receptor in hematologic malignancies ........................... 28

1.4.3 Leptin/leptin receptor in other stem cells ............................................. 34

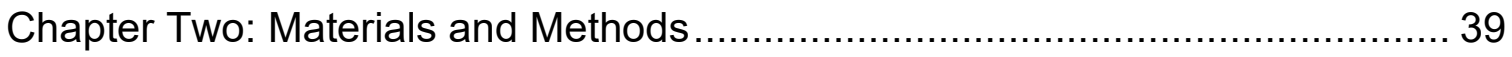

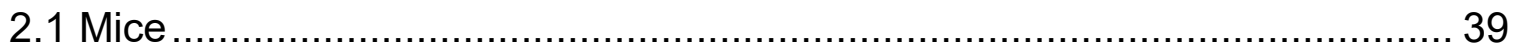

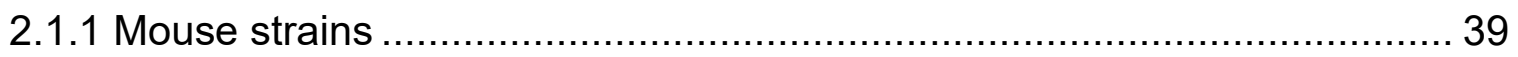

2.1.2 Mouse irradiation for transplantations .............................................. 39

2.2 Preparation of cells from fresh bone marrow ........................................ 40

2.3 Enrichment of mouse Lin- BM cells and hCB CD34 ${ }^{+}$cells ........................ 40

2.4 Cell staining for flow cytometry analyses and sorting …......................... 40

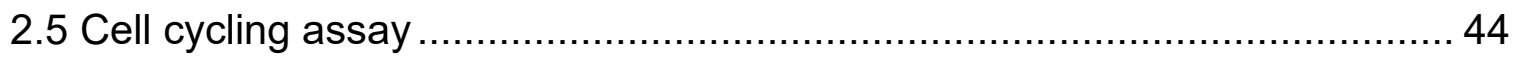

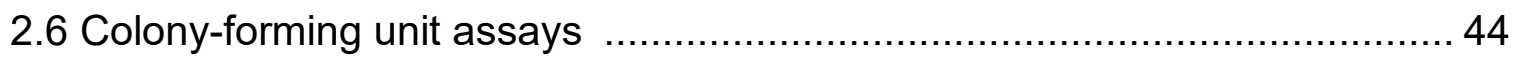

2.6.1 High specific activity tritiated thymidine kill assay ................................. 44

2.7 Competitive transplantation assays to assess engraftment and competitive

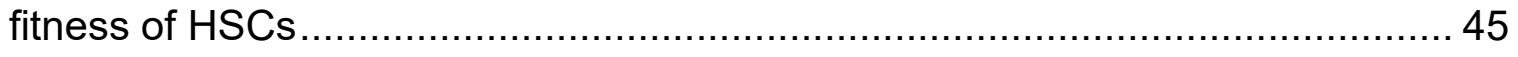

2.7.1 Limiting dilution assays to calculate numbers of functional HSCs ............ 46

2.7.1.1 Competitive repopulating units (CRUs), measures of mouse BM

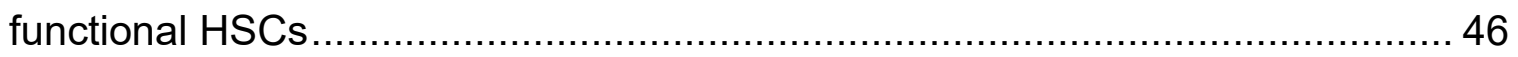

2.7.1.2 SCID-repopulating cells (SRCs), measures of human CB functional

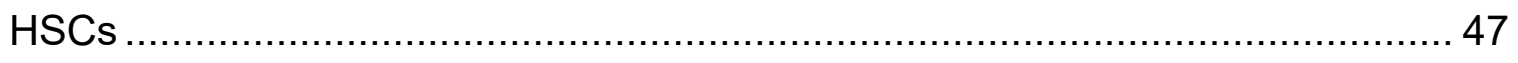

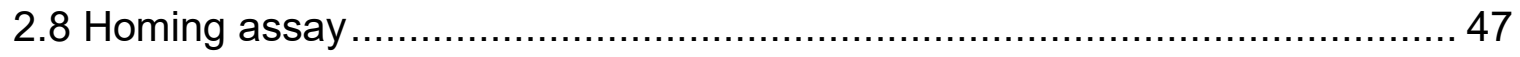

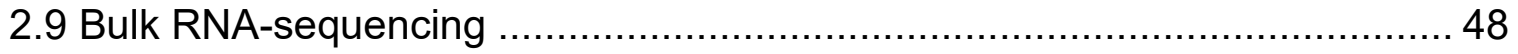

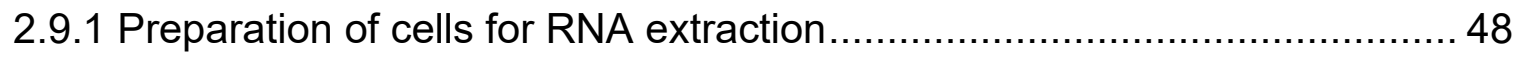

2.9.2 Library preparation, data collection and analyses............................... 48 
2.10 Statistical analyses

Chapter Three: LEP/LEPR signaling is not required for HSC/HPC maintenance under homeostasis, but LEPR-expressing HSCs represent a subset of highly engrafting long-term functional murine HSCs.

3.1 $\mathrm{LEPR}^{+}$cells are significantly outnumbered by LEPR ${ }^{-}$cells in young adult C57BL6/J WT mouse BM

3.2 Germline knockout of murine Lep or Lepr did not alter numbers of BM phenotypic HSCs/HPCs under homeostasis 54

3.3 LEPR+LSK cells were more highly enriched for phenotypically defined HSCs and MPPs 57

3.4 Compared to LEPR-LSK cells, LEPR+LSK cells contained significantly higher numbers of both colony-forming progenitor cells and functional long-term self-renewing HSCs 61 3.5 LEPR differentiated SLAM HSCs into two functionally distinct populations with LEPR ${ }^{+} H S C s$, a smaller subset but with significantly higher repopulating potential

3.6 LEPR ${ }^{+} H S C$ s did not exhibit a lineage bias in competitive transplantation assays, shared similar homing capacity and cycling status with LEPR-HSCs .... 80 3.7 LEPR ${ }^{+} H S C s$ constitute a subset of functional long-term repopulating HSCs that is characterized by a pro-inflammatory transcriptomic signature 89 3.8 Human cord blood LEPR ${ }^{+}$CD $34^{+}$cells, a minor subset of total CD $34^{+}$ cells, were more highly enriched for phenotypically defined HSCs and showed a trend to enhanced engraftment compared to LEPR-CD34 ${ }^{+}$ 
cells in NSG mice

3.9 Phenotypic characterizations of LEPR ${ }^{+}$hematopoietic stem and progenitor cells in middle-aged mice as compared to LEPR- cells ................................. 107

Chapter Four: Discussion and future directions............................................ 118

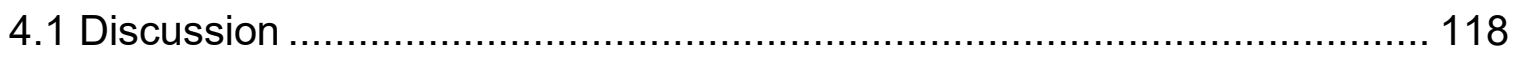

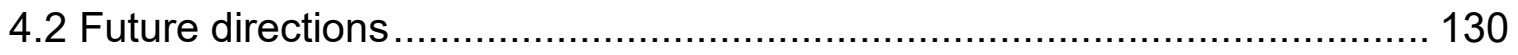

4.2.1 Role(s) of LEP/LEPR in hematopoiesis under non-pathological

conditions

4.2.2 Role(s) of LEP/LEPR in hematopoiesis under pathological/leukemic

conditions

4.3 Conclusion

References. 138

Curriculum Vitae 


\section{LIST OF TABLES}

Table 1.1 LEP/LEPR in hematopoiesis. What is known? ........................ 31

Table 2.1 List of fluorophore conjugated antibodies used in the study ..............42

Table 4.1 List of major findings (Trinh et al., 2020)............................. 121

Table 4.2. LEP/LEPR in hematopoiesis. What's next? ............................ 134 


\section{LIST OF FIGURES}

Figure 3.1_BM LEPR ${ }^{+}$cells are significantly outnumbered by LEPR- cells ....... 52 Figure 3.2_Germline Knockout of murine Lep or Lepr did not alter numbers of BM phenotypic HSCs/HPCs

Figure 3.3 LEPR ${ }^{+}$LSK cells were more highly enriched for phenotypically defined HSCs as compared to LEPR-LSK cells

Figure 3.4 Compared to LEPR'LSK cells, LEPR+LSK cells contained significantly higher numbers of both colony-forming progenitor cells.

Figure 3.5 Compared to LEPR-LSK cells, LEPR+LSK cells contained significantly higher numbers of both colony-forming progenitor cells and functional long-term HSCs.

Figure 3.6 LEPR ${ }^{+}$LSK cells contained significantly higher numbers of competitive repopulating units (CRUs) as compared to LEPR-LSK cells in a limiting dilution assay transplant.

Figure 3.7 Compared to LEPR-LSK cells, LEPR+LSK cells were more highly

enriched for self-renewing HSCs as shown in secondary transplants

Figure 3.8 LEPR differentiates SLAM HSCs into two functionally distinct populations with LEPR ${ }^{+}$HSCs, a smaller subset but with significantly higher

repopulating potential

Figure 3.9 BM from recipient mice transplanted with LEPR+HSCs were significantly enriched with HSCs and HPCs 78

Figure 3.10 LEPR+HSCs had a similar lineage output with LEPR-HSCs 81

Figure 3.11 LEPR ${ }^{+}$HSCs homed to the BM niche at a similar capacity and 
shared similar levels of CXCR4 expression with LEPR-HSCs

Figure 3.12 LEPR $^{+}$HSCs shared similar cycling status with LEPR-HSCs

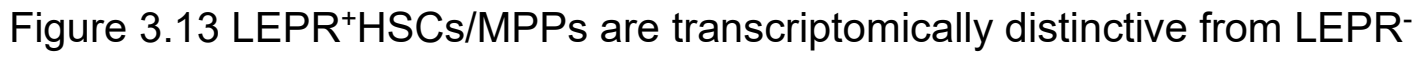

HSCs/MPPs.

Figure 3.14 LEPR ${ }^{+}$HSC/MPP expressed predominantly the short isoforms

Lepr-a and Lepr-c but not the long isoform Lepr-b

Figure 3.15 LEPR ${ }^{+} \mathrm{HSCs}$ were characterized by proinflammatory

transcriptomic pathways, whereas LEPR-HSCs were enriched for genes

involved in mitochondrial membrane protein and respiratory electron

transport chain

Figure 3.16 LEPR ${ }^{+} H S C s$ were enriched for genes associated with long-term HSCs, whereas LEPR-HSCs were enriched for an intermediate progenitor

gene profile.

Figure 3.17 Human CB (hCB) LEPR ${ }^{+} \mathrm{CD} 34^{+}$cells, a minor subset of total

CD34 ${ }^{+}$cells, were more highly enriched for phenotypically defined HSCs 102

Figure 3.18 hCB LEPR ${ }^{+} \mathrm{CD} 34^{+}$cells showed a trend to enhanced engraftment compared to LEPR-CD34+ ${ }^{+}$cells in NSG mice.

Figure 3.19 Representative plots of HSCs in middle-aged mice in comparison to young mice

Figure 3.20 With age, fractions of long-term LEPR ${ }^{+}$SLAM HSCs expanded over LEPR- SLAM HSCs

Figure 3.21 With age, fractions of total MPP and different lineage-biased MPP subsets expanded over LEPR- populations 
Figure 3.22 Unlike stem cells, LEPR ${ }^{+}$progenitor populations did not expand with aging and remained as low as in young BM

Figure 4.1 LEPR ${ }^{+}$HSCs under steady state condition becomes 'activated' in post-injury recovery (hypothetical) 127 


\section{LIST OF PICTURES}

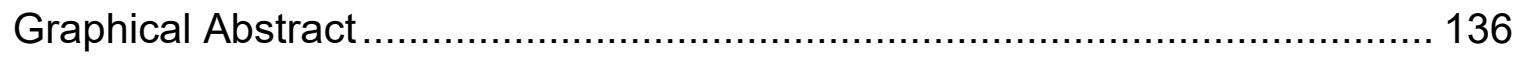




\section{LIST OF ABBREVIATIONS}

\begin{tabular}{|l|l|}
\hline AGM & Aorta-genital ridge-mesonephros \\
\hline ALL & Acute lymphoblastic leukemia \\
\hline AML & Acute myeloid leukemia \\
\hline ANGPT1 & Angiopoietin 1 \\
\hline BCSC & Breast cancer stem cell \\
\hline BFU-E & Burst-forming unit-erythroid \\
\hline BM & Bone marrow \\
\hline CB & Cord blood \\
\hline CFU & Colony-forming unit \\
\hline CFU-GEMM & CFU-granulocyte, erythrocyte, macrophage, megakaryocyte \\
\hline CFU-GM & CFU-granulocyte,macrophage \\
\hline CH & Clonal hematopoiesis \\
\hline CHIP & Clonal hematopoiesis of indeterminate potential \\
\hline CLP & Common lymphoid progenitor \\
\hline CMP & Common myeloid progenitor \\
\hline CPT1 & Carnitinepalmitoyl transferase 1 \\
\hline CRU & Competitive repopulating unit \\
\hline CSC & Cancer stem cell \\
\hline DPP4 & Dipeptidyl peptidase 4 \\
\hline E & Embryonic \\
\hline EC & Endothelial cell \\
\hline
\end{tabular}




\begin{tabular}{|l|l|}
\hline FACS & Fluorescence activated cell sorting \\
\hline FAO & Fatty acid oxidation \\
\hline G-CSF & Granulocyte colony stimulating factor \\
\hline GF & Growth factor \\
\hline GFP & Green fluorescence protein \\
\hline GM-CSF & Granulocyte-macrophage colony-stimulating factor \\
\hline GMP & Granulocyte macrophage progenitor \\
\hline GVHD & Graft-versus-host disease \\
\hline H & Human \\
\hline HCT & Hematopoietic cell transplantation \\
\hline HLA & Human Leukocyte Antigen \\
\hline HPCs & Hematopoietic progenitor cells \\
\hline HPE & Homeostatic peripheral T cell expansion \\
\hline HSCs & Hematopoietic stem cells \\
\hline IL & Interleukin \\
\hline iPSC & Induced pluripotent stem cell \\
\hline LDA & Limiting dilution assay \\
\hline LEPR & Leptin receptor \\
\hline LEPTIN & Leptin \\
\hline LIF & Leukemia inhibitory factor \\
\hline LSK & MDS \\
\hline
\end{tabular}




\begin{tabular}{|l|l|}
\hline MEP & Megakaryocyte erythrocyte progenitor \\
\hline MHC & Major Histocompatibility Complex \\
\hline MI & Myocardial infarction \\
\hline mPB & Mobilized peripheral blood \\
\hline MPP & Multipotent progenitor \\
\hline MSC & Mesenchymal stem/stromal cell \\
\hline OPN & Osteopontin \\
\hline PMF & Primary myelofibrosis \\
\hline PTN & Pleiotrophin \\
\hline ROS & Reactive oxygen species \\
\hline Sca-1 & Stem cell antigen 1 \\
\hline SCF & Stem cell factor \\
\hline SDF-1 & Stromal cell-derived factor \\
\hline SRC & SCID-repopulating cell \\
\hline ST & Short-term \\
\hline TBI & Total body irradiation \\
\hline TF & Transcription factor \\
\hline TNFa & Wild-type \\
\hline WT & \\
\hline
\end{tabular}




\section{CHAPTER ONE: Introduction}

\subsection{Hematopoietic cell transplantation}

The first hematopoietic cell transplantation (HCT) was performed by Dr. E.

Donnall Thomas in the late 1950s for a patient with end-stage acute leukemia. It was a syngeneic HCT in which the patient received marrow from her identical twin after receiving total-body irradiation (Thomas, Lochte, Cannon, Sahler, \& Ferrebee, 1959). This happened after observational studies that showed successful engraftment of bone marrow (BM) cells in myelosuppression (Barnes, Corp, Loutit, \& Neal, 1956; Lorenz, Uphoff, Reid, \& Shelton, 1951). Since then, HCT has become an invaluable curative regimen for a variety of diseases including malignant and non-malignant conditions (Khaddour, Hana, \& Mewawalla, 2020).

Allogeneic HCT became more feasible upon the discovery of Human Leukocyte Antigens (HLA) in the early 1960s (Dausset, 1958); they are proteins expressed on the surface of cells that determine whether donor cells are immunologically suitable and compatible for patients. HLA can be divided into two groups. (HLAA, B, and C), encoded by the Class I Major Histocompatibility Complex (MHC), are expressed by most cell types in the body and are recognized by $\mathrm{CD} 8^{+} \mathrm{T}$ cells. Class II MHC encodes HLA- DP, DQ and DR, which are expressed on antigen-presenting cells and recognized by $\mathrm{CD} 4^{+} \mathrm{T}$ cells. Based on this matching system, there are three main types of HCT - autologous transplantation (donor 
cells are patient's own cells), syngeneic transplantation (donor cells come from a monozygotic twin) and allogenic transplantation (donor cells are from an HLA matched family member, an unrelated matched donor or partially mismatched family member also called haploidentical) (Khaddour et al., 2020).

Like other types of treatments, HCT has potential complications and can be lifethreatening in some cases. Depending on several factors such as sources of transplanted cells, the preparative regimen and age of patients, complications can be acute (within 90 days post-transplant) or chronic. Common acute complications include insufficient blood cell production (neutropenia, thrombocytopenia, anemia), various infections, mucositis and sinusoidal obstruction syndrome. Graft-versus-host disease (GVHD) can occur in the acute phase or chronically. Other chronic complications include infections with Varicella Zoster virus and encapsulated bacteria (Khaddour et al., 2020).

\subsubsection{Sources of transplantable cells - advantages and disadvantages}

Successful clinical outcomes of HCT are largely determined by the engraftment of rare populations of cells called hematopoietic stem cells (HSCs) and hematopoietic progenitor cells (HPCs). In the adult hematopoietic hierarchy, HSCs are the only blood cell type that satisfy the definition of adult somatic stem cells, which include multipotency (the ability to give rise to all mature blood and immune cells) and self-renewal (the ability to generate more HSCs without differentiation) (Seita \& Weissman, 2010). Meanwhile, multipotent progenitor 
(MPP) cells can differentiate to more mature functional cells but cannot selfrenew. As MPPs differentiate descending the hierarchy, the daughter progenitor cells become more and more lineage-restricted. These cells can be collected from three main sources - bone marrow (BM), mobilized peripheral blood (mPB) and cord blood (CB). Each of these has their own specific advantages and disadvantages.

Studies comparing BM vs. mPB HSCs showed that mPB HSCs had a faster immune reconstitution rate (Bensinger et al., 2001; Blaise et al., 2000; Powles et al., 2000). However, patients who received mPB transplants had higher incidences of chronic GVHD (Anasetti et al., 2012; Bensinger et al., 2001) while acute GVHD was similar between the two groups in HLA- identical matched related donors (Bensinger et al., 2001). Particularly for patients with hematological malignancies, most randomized controlled trials comparing matched related donor BM and mPB did not find significant differences in terms of relapse, transplant-related GVHD and mortality, disease-free survival and overall survival (Blaise et al., 2000; Powles et al., 2000). On the other hand, compared to both BM and mPB HSCs, CB HSCs (found in a population of CD34 ${ }^{+}$ cells) do not require as stringent HLA matching. Cryopreserved CB units are more readily available through $\mathrm{CB}$ banks, and have less risk of infections as well as relatively lower incidences of GVHD, which allows use of partially disparate HLA CB units for HCT (Broxmeyer, 2008; Copelan, 2006) . However, this method suffers from a delay in engraftment of neutrophils, platelets and immune cell 
recovery with somewhat higher chances of disease relapses, and the limited numbers of HSCs per CB unit is also an issue for larger children and adult patients (Broxmeyer, 2008; Khaddour et al., 2020).

\subsubsection{Efforts to improve clinical outcomes for patients receiving HCT}

One of the biggest challenges with allogeneic HCT is T-cell immune

reconstitution, which may take a long time (months to years) and is highly varied among patients. As a result, these patients may be at higher risk to develop disease relapse or have higher HCT-related morbidity and mortality rate (de Koning, Nierkens, \& Boelens, 2016).

Several approaches including those for pre-HCT, peri-HCT and post-HCT have been taken in order to overcome this problem. For example, a pre-HCT strategy is to increase the cell dose transplanted because T-cell recovery needs to be preceded by myeloengraftment; hence, a sufficiently high cell number will speed up the process. Likewise, the sources of cells also matter - mPB HCT without ex vivo T-cell depletion generally has a faster $\mathrm{T}$ cell recovery rate compared to BM HCT (Ottinger, Beelen, Scheulen, Schaefer, \& Grosse-Wilde, 1996; Storek et al., 2001). During the peri-HCT phase, some studies have suggested that physical exercise (Hayes, Rowbottom, Davies, Parker, \& Bashford, 2003) and adequate specific nutrient supplementations (iron, selenium, zinc and antioxidant vitamins) (Cunningham-Rundles, McNeeley, \& Moon, 2005; Worthington \& Cresci, 2011) are important for IR post allogeneic HCT. Several approaches have been 
investigated to promote T-cell recovery in the post-transplantation period; these either target homeostatic peripheral T cell expansion (HPE) or thymopoiesis. IL-2 cytokine treatment is introduced to induce $\mathrm{T}$ cell differentiation and proliferation (Inamoto et al., 2011; Kennedy-Nasser et al., 2014); similarly, IL-15 enhances HPE by increasing B and T cell expansion (Alpdogan et al., 2005). To promote thymopoiesis, treatment with IL-17 increased numbers of functional T cells by inducing cell differentiation and proliferation of naïve T cells. It also enhanced T cell receptor diversity, which is required for thymopoiesis (Perales et al., 2012). In addition, sex steroid ablation (Sutherland et al., 2008), treatments with keratinocyte growth factor (Min et al., 2002) or thymosin $\alpha 1$ (Perruccio et al., 2010) were found to be useful to promote T cell maturation and regeneration.

To quicken the time to hematopoietic recovery, particularly in the case of $\mathrm{CB}$ HCT, substantial efforts have been made over decades to increase HSC and HPC numbers through ex vivo expansion and/or to enhance the ability of transplanted cells to home to the BM. The BM is the site where the engrafted cells are nurtured for proliferation, survival, self-renewal, and differentiation, and since the homing of HSCs is not absolute, the enhancement of having the infused cells reach the BM in higher numbers allows for more rapid engraftment (Huang, Guo, Capitano, \& Broxmeyer, 2019; Huang, Guo, Liu, Wan, \& Broxmeyer, 2018). 
Compound screening has yielded numerous exciting results. Small molecules such as UM171 (Fares et al., 2014), StemRegenin 1(SR1) (Boitano et al., 2010), valproic acid (VPA) (Chaurasia, Gajzer, Schaniel, D'Souza, \& Hoffman, 2014) were found to expand human HSCs/HPCs several folds. Both SR1 (Wagner et al., 2016) and UM171 (Cohen et al., 2020) have been tested in clinical trial phase I/II. Notably, neither SR1 or UM171 expanded mouse HSC, so it's difficult to investigate their mechanisms in vivo using murine models. Likewise, treatment with recombinant HOXB4 (or its overexpression) induced both human $\mathrm{CD}_{4} 4^{+}$and mouse HSC expansion (Antonchuk, Sauvageau, \& Humphries, 2002; Huang et al., 2016; Krosl et al., 2003). One issue with ex vivo experiments is that the specific culture conditions matter. Some compounds while being able to expand HSCs will not show its effect in certain culture media, like retinoid acid receptor (RAR) or peroxisome proliferator-activated receptor (PPAR) gamma antagonists when cultured in StemSpan ${ }^{\mathrm{TM}}$ Serum-Free Medium (Guo, Huang, Lee, Lee, \& Broxmeyer, 2018).

For HSCs to migrate and lodge into the BM niche, a key interaction needs to occur between CXCR4, a G-protein-coupled chemokine receptor expressed on the surface of HSCs, and chemokine ligand CXCL12/stromal cell-derived factor (SDF)-1 that is secreted as a gradient to provide HSCs directional cues (Dar et al., 2011; C. H. Kim et al., 1999; Lapidot \& Kollet, 2002; Q. Ma et al., 1998; Sharma, Afrin, Satija, Tripathi, \& Gangenahalli, 2011; Zou, Kottmann, Kuroda, Taniuchi, \& Littman, 1998). To enhance homing of HSCs to the BM upon 
transplantation, there have been various strategies, which targets three main cellular compartments - cell membrane, cytoplasm and nucleus (Huang et al., 2019). A very simple and non-invasive approach was to warm the cells for a short time (4 hours) that resulted in lipid rafts formation allowing CXCR4 to aggregate and hence more likely to interact with CXCL12 (M. L. Capitano, Hangoc, Cooper, \& Broxmeyer, 2015). The plasma membrane is home to different enzymes that can modulate CXCR4-CXCL12 interaction such as dipeptidyl peptidase 4 (DPP4). CXCL12 can be truncated by DPP4 and while truncated CXCL12 is much less active than full length CXCL12, it strongly blocks the activity of full length CXCL12 (Christopherson, Hangoc, \& Broxmeyer, 2002) interferes with the full-length ligand activity. Hence, inhibition of DPP4 has been shown to promote HSC homing and engraftment (Christopherson, Hangoc, Mantel, \& Broxmeyer, 2004). More recently, nuclear receptor modulators including but not limited to gluococorticoid receptor (GR) (Guo, Huang, Cooper, \& Broxmeyer, 2017; Tan \& Wahli, 2016) and histone deacetylase (HDAC) 5 inhibition (Huang et al., 2018) upregulate CXCR4 expression thereby enhancing homing and engraftment of HSCs.

Moreover, it is well-appreciated that HSCs/HPCs are nurtured in a hypoxic BM microenvironment ( $15 \%$ oxygen as compared to $21 \%$ in ambient air); hence, methods for isolation, processing and ex vivo culturing that can protect HSCs from the differentiation-inducing effects of hyperoxic reactive oxygen species 
(ROS) preserve HSC functions and numbers (Huang et al., 2019; Mantel et al., 2015)

\subsection{Overview of murine hematopoiesis}

To meet a tremendous demand of daily blood cell production, millions of mature blood cells per day, hematopoiesis needs to be tightly orchestrated over the lifetime of an organism (Dzierzak \& Medvinsky, 1995; Silva, Anderson, \& Gatenby, 2011). The entire hematopoietic system relies on a very small number of cells namely HSCs. Through a series of more differentiated precursors, HSCs give rise to all types of mature blood and immune cells in the body (Rieger \& Schroeder, 2012). HSCs and HPCs first emerge during embryonic development in the yolk sac and the embryonic mesoderm, then migrate to and expand in fetal liver, and eventually reside mostly in the adult BM where they interact with a complex network of niche cells and noncellular factors (A. L. Medvinsky, Samoylina, Muller, \& Dzierzak, 1993; Morrison \& Scadden, 2014).

HSCs and HPCs are by far the most studied adult stem cells for many reasons they can be physically isolated without significant stress and collected with adequate numbers, they can be identified and assessed phenotypically through markers, and studied functionally in vitro and in vivo at a single cell level (Rieger \& Schroeder, 2012). The murine hematopoietic system is arguably the best understood system. Early quantitative studies in the field have laid the foundation for modern HSC research (Becker, Mc, \& Till, 1963; Siminovitch, McCulloch, \& 
Till, 1963; Till, 1961; Till \& Mc, 1961). The evolution of different techniques allowed studies of lineage tracing (Sun et al., 2014), timing and site specificity of cell development and sophisticated functional behaviors in vivo including engraftment of human HSCs in immune-compromised humanized mouse models (Abarrategi et al., 2018; Bock, Orlic, Dunbar, Broxmeyer, \& Bodine, 1995;

McDermott, Eppert, Lechman, Doedens, \& Dick, 2010; Vormoor et al., 1994). Excitingly, with the advancement of bioinformatic technology, analyses of large datasets have shed light into the molecular, genetic and epigenetic regulations of HSCs both in the context of normal and malignant hematopoiesis (Nobile et al., 2019; Pellin et al., 2019; Zeng et al., 2019).

\subsubsection{Emergence of HSCs during embryonic hematopoiesis}

During embryogenesis, the hematopoietic system is derived from the mesoderm, which is formed in the primitive-streak-stage at 6.5 days postcoitum (days after conception referring to the age of an embryo). Similar to avian and amphibian systems, in mouse there are two embryonic sites of hematopoietic activity before fetal liver hematopoiesis - the ventral compartments (yolk sac) and the dorsal compartments (intraembryonic aorta, genital ridge and mesonephros, also known as the AGM region) (Dzierzak \& Medvinsky, 1995). It is generally accepted that hematopoietic activities in the yolk sac and the AGM happen independently from one another (A. Medvinsky, Rybtsov, \& Taoudi, 2011). Notably, hematopoietic activity in the yolk sac is transient (so called the first wave - primitive hematopoiesis) while HSCs and HPCs produced intraembryonically will take 
residence in definitive hematopoietic organs (also known as the second wave definitive hematopoiesis) (A. Medvinsky et al., 2011).

Blood islands first appear in the extraembryonic yolk sac as early as embryonic (E) day 7 (E7) and generate mainly primitive nucleated erythrocytes and to a lesser extent granulocytes and macrophages (Godin, Dieterlen-Lievre, \& Cumano, 1995; Moore \& Metcalf, 1970). These are followed with lymphoid activity of T and B cells around E8 - 9 (Liu \& Auerbach, 1991; Ogawa et al., 1988) and colony forming units-spleen (CFU-S; more mature subset of HSCs) at late E9 (A. L. Medvinsky et al., 1993). Meanwhile, the AGM begins to form at E8 and gives rise to more potent CFU-S activity than the yolk sac beginning at E9.5 (A. L. Medvinsky et al., 1993). T and B cells are also found in the paraaortic splanchnopleure (P-Sp) in E8 - 9 embryos. Finally, the long-term repopulating HSCs arise at late E10 in the AGM (A. L. Medvinsky, Gan, Semenova, \& Samoylina, 1996; Muller, Medvinsky, Strouboulis, Grosveld, \& Dzierzak, 1994). Hence, consistent with earlier observations in quail chick chimeric embryos (Dieterlen-Lievre, 1975), definitive HSCs (dHSCs) have an intraembryonic origin. However, in contrast to avian and similar to human, mouse dHSCs do not emerge from blood islands in the region of dorsal aorta but rather intravascular region of the aortic hemogenic endothelium and postumbilical arteries (GarciaPorrero, Godin, \& Dieterlen-Lievre, 1995). 
There are several evidences that pointed to the crucial role of the AGM region in generating definitive adult HSCs autonomously (A. L. Medvinsky et al., 1996). Explants from E10.5 and E11.5 AGMs can grow and expand HSCs, respectively (Kumaravelu et al., 2002). Similarly, HSCs proliferate in the presence of growth factors when cultured with explanted AGMs (Robin et al., 2006; Taoudi et al., 2008). In transplantation assays, while yolk sac explants only give rise to shortterm erythromyeloid engraftment, E8 para-aortic splanchnopleura explants (prior to established circulation) can generate long-term engraftment of lymphomyeloid cells (Cumano, Ferraz, Klaine, Di Santo, \& Godin, 2001). Moreover, the placenta has also been suggested as another site of definitive hematopoiesis. Placenta is believed to contain dHSCs concomitantly with the AGM region by E10.5 - 11, as shown in transplantation assays (Gekas, Dieterlen-Lievre, Orkin, \& Mikkola, 2005).

Mechanistically, the two key transcriptional regulators of dHSC specification and emergence from the AGM are RUNX1 and $\mathrm{NOTCH}$, and they have slightly differential roles. Both RUNX1 and NOTCH (also NOTCH targets and ligands) are expressed by intra-aortic hemogenic clusters (North et al., 1999; RobertMoreno, Espinosa, de la Pompa, \& Bigas, 2005). Studies have shown that Notch1\% E9.5 yolk sac and P-Sp cells failed to initiate adult hematopoiesis when injected into newborn recipients (Kumano et al., 2003). In parallel, Runx $1^{-1-}$ mouse embryos do not develop clonogenic progenitors and dHSCs and die by E12.5 even though yolk sac erythropoiesis remains close to normal (Yokomizo et 
al., 2008). Unlike Runx1\% mutants, Notch $1^{1-}$ embryos have normal clonogenic potential in the yolk sac, which suggested that NOTCH1 signaling is specifically important for AGM hematopoiesis (Bertrand, Cisson, Stachura, \& Traver, 2010). In addition, other molecular factors such as bone morphogenetic protein, soluble hedgehog, vascular endothelial growth factor as well as nitric oxide signaling and shear stress also play a significant role in the induction of hemogenic fate in the embryonic AGM (A. Medvinsky et al., 2011).

\subsubsection{Definitive (adult) hematopoiesis}

\subsubsection{Adult HSCs and HPCs}

A few weeks after birth, dHSCs undergo a dramatic phenotypic switch from fetal HSCs, which are highly proliferative and engraft robustly with faster kinetics in transplantation assays, to adult HSCs, which are mostly quiescent and reside in the hypoxic niche of the BM microenvironment (Bowie et al., 2007). Positioned at the apex of the hematopoietic hierarchy, HSCs are the only type of blood cells that are capable of long-term self-renewal and differentiation into all lineages of blood and immune cells. Stepwise identifications using multiple surface markers allows isolation of different HSCs and HPCs using fluorescence activated cell sorting (FACS). In general, more potent repopulating cells express stem cell antigen (Sca-1) and the receptor for stem cell factor (SCF) CD117 (c-Kit) while they express low levels of or are negative for mature cell markers (lineage markers) (Okada et al., 1992; Spangrude, Heimfeld, \& Weissman, 1988). These 
are called LSK cells, which contain a mixture of different HSC and HPC populations.

Based on that foundation, different systems of more refined markers are utilized to specifically identify prospective HSCs and multipotent progenitors (MPPs). HSCs can be obtained using Mac-1 and CD4 expression levels (HSCs defined

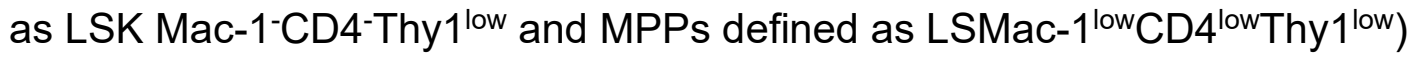
(Morrison, Wandycz, Hemmati, Wright, \& Weissman, 1997; Morrison \& Weissman, 1994). More purified HSCs are also found in LSK cells that are CD34 negative/low (Osawa, Hanada, Hamada, \& Nakauchi, 1996), Flt3 negative (Adolfsson et al., 2005; Christensen \& Weissman, 2001; L. Yang et al., 2005) or have increased efflux of Hoechst dye 33342 (Goodell, Brose, Paradis, Conner, \& Mulligan, 1996) or low staining for Rhodamine123 dye (Bertoncello, Hodgson, \& Bradley, 1985). More recently, highly purified functional HSCs and MPPs have been identified using the SLAM family markers CD150 (SLAMF1) and CD48 (SLAMF2) - HSCs are defined as LSKCD $150^{+}$CD48 and MPPs are defined as LSKCD150+CD48- (Forsberg et al., 2005; Kiel et al., 2005; I. Kim, He, Yilmaz, Kiel, \& Morrison, 2006; O. H. Yilmaz, Kiel, \& Morrison, 2006). Notably, SLAM HSCs can be further divided into two functionally distinct subsets using Leptin Receptor (LEPR) (Trinh et al., 2020). LEPR+SLAM HSCs engrafted more robustly than LEPR- cells. In fact, LEPR ${ }^{+}$LSK cells contained most HSC activities upon transplantation as compared to LEPR-LSK cells. 
Given the extraordinarily crucial roles that HSCs play in both hematopoiesis and immunity, their fate decisions - self-renewal versus differentiation, survival, proliferation versus quiescence, mobilization versus homing, lineage choices are constantly under strict regulation. To maintain adequate hematopoietic output under homeostasis as well as during stressful conditions, HSCs need to balance between self-renewal and differentiation. Uncontrolled differentiation or inadequate self-renewal can lead to exhaustion of the HSC pool; in contrast, a block in differentiation or abnormally increased self-renewal may result in myeloproliferative malignancies. These fate decisions are influenced by both extrinsic mechanisms (BM microenvironment) (will be discussed in more details in section 1.2.2.2) and intrinsic programs (Rieger \& Schroeder, 2012).

For a multipotent cell to commit to a specific cell lineage, there needs to be a change in global gene expression - upregulation of that lineage-specific programs accompanied by downregulation of genes associated with other lineages; this process is orchestrated by activities of different molecular networks including transcription factors (TFs), transcriptional cofactors, chromatin modifiers and other regulatory factors (Hamey et al., 2017). For example, while GATA2 is highly expressed in early progenitors, a switch from GATA2 to GATA1 mediated by increased interaction between GATA1 and FOG1 and hence displacement of GATA2 from its own upstream enhancer initiates erythropoiesis (Grass et al., 2003). Furthermore, it has been well-recognized that HSCs are comprised of heterogenous populations even at molecular levels (R. Lu, 
Czechowicz, Seita, Jiang, \& Weissman, 2019) with lineage-specific subsets, and these lineage biases are influenced by microenvironmental niche factors in the BM (Pinho et al., 2018). Similarly, myeloid versus lymphoid biased subsets of MPPs have been well-characterized phenotypically and functionally (Pietras et al., 2015).

\subsubsection{Adult bone marrow niches}

Most of the knowledge about BM niches come from studies of mouse long bones. BM is a highly dynamic and intricate organ. Proposed by R. Schofield in 1978, the concept of BM niche refers to the regulatory unit that is required to maintain HSC self-renewal and differentiation (Schofield, 1978). BM niches can be subdivided into two major categories - the endosteal niche and the vascular niche. Understanding how different cellular (hematopoietic and nonhematopoietic cells) components and growth factors regulate HSC functions can have critical implications for the advancements in the field as well as clinical applications.

Early studies suggested that HSCs and HPCs are more populated in the endosteal region of the bone (Gong, 1978; B. I. Lord, Testa, \& Hendry, 1975). In addition, upon transplantation into recipients either with (Lo Celso et al., 2009; Xie et al., 2009) or without (Nilsson, Johnston, \& Coverdale, 2001) myeloablation, HSCs tend to home near the endosteal surface. In fact, HSCs can be expanded by treating mice with parathyroid hormone, a regulator of bone 
turnover, mediated partially through enforced Notch signaling (Calvi et al., 2003). Furthermore, treatment of mice expressing a truncated form of the herpesvirus thymidine kinase gene under the control of rat type I collagen with ganciclovir to induce osteoblast ablation reduced the numbers of myeloid, erythroid and lymphoid progenitors (Visnjic et al., 2004).

Unlike the early findings, discoveries of SLAM HSC markers and advancement in microscopic imaging have revealed that under homeostasis HSCs are localized around the sinusoid instead of the endosteum, hence the vascular niche. One of the major critical cellular components of the vascular niche are BM-derived mesenchymal stem/stromal cells (MSCs), which are capable of self-renewing and can potentially differentiate into osteoblasts, adipocytes and chondrocytes (B. O. Zhou, Yue, Murphy, Peyer, \& Morrison, 2014). Interestingly, both human and mouse MSCs when transplanted subcutaneously (Sacchetti et al., 2007) or into the renal capsule (Chan et al., 2009) (respectively) of recipient animals form an active hematopoietic niche.

MSCs consists of different cell types, and each of them has been demonstrated to play a significant role in maintaining hematopoiesis under steady-state and some in emergency (stressed) hematopoiesis. One subset of MSCs are perivascular Nestin (Nes) ${ }^{+}$cells. These cells are closely associated with the sympathetic nerves that innervate the BM. Like other tissues in the body, HSCs are subjected to circadian rhythm regulation in which they are mobilized into the 
circulation mediated by actions of the sympathetic nervous system on CXCL12 expression (Katayama et al., 2006; Mendez-Ferrer, Lucas, Battista, \& Frenette, 2008). Nes+MSCs play an important role in secreting HSC-maintaining factors including CXCL12 and SCF. They are also a source of angiopoietin 1 (ANGPT1), osteopontin (OPN) and interleukin-7 (IL-7), which is crucial for homeostasis of lymphoid progenitors and mature B cells (Mendez-Ferrer et al., 2010). Another important subpopulation of MSCs are CXCL12-abundant reticular (CAR) stromal cells, which are found mostly around sinusoid and secrete SCF as well as IL-7 (Omatsu et al., 2010). On the other hand, BM adipocytes, also derived from MSCs, are suggested to negatively regulate hematopoiesis (Naveiras et al., 2009). Adipocyte-derived adiponectin inhibits HPC proliferation in vitro (Yokota et al., 2000). Upon stress such as post-irradiation adipocytes become abundant and enhance HSC recovery by secreting SCF (B. O. Zhou et al., 2017). Among the different growth factors, SCF plays an indispensable role in maintaining HSC and HPC pools. A mouse model with GFP knock-in at the Scf locus revealed two crucial sources of BM SCF, which are endothelial cells (ECs) and LEPRexpressing stromal cells (Ding, Saunders, Enikolopov, \& Morrison, 2012). In addition, both of these cell types also secrete pleiotrophin (PTN) that are required for HSC homeostasis, while PTN from endothelial cells is critical for the recovery of long-term HSCs upon stress (Himburg et al., 2018). 


\subsection{Hematopoiesis during ageing and increased risks for malignancies}

It is well-documented that aging is associated with accumulations of somatic mutations ubiquitously present throughout different organs (Hoang et al., 2016; Martincorena \& Campbell, 2015; Starling, 2018; Welch et al., 2012). These ageassociated mutations can be subdivided into a few major categories; all of them have been suggested to happen frequently with ageing. 1) The most prevalent one is the spontaneous deamination of 5-methylcytosine to thymine typically on CpG dinucleotides (Duncan \& Miller, 1980); 2) In DNA double-strand breaks nonhomologous end joining occurs as an attempt to fix the damages and may be prone to small insertions or deletions (also called indels); 3) Replication errors by DNA polymerase can also introduce mismatched base substitutions or indels; 4) Larger sized structural variations such as rearrangements or loss of big segments of chromosomes.

Under certain selection pressure, some of these mutations may grant HSCs a fitness advantage and allow them to outgrow others, creating what is called clonal hematopoiesis $(\mathrm{CH})$ (Jaiswal \& Ebert, 2019). CH is characterized by the overrepresentation of blood cells derived from a single clone regardless of cause or disease state. Early evidences of $\mathrm{CH}$ came from the observation of TET2 mutations in approximately $5 \%$ of healthy elderly females with nonrandom Xchromosome inactivation without cancer (Busque et al., 2012). Furthermore, patients after being treated for acute myeloid leukemia $(A M L)$ were found to harbor leukemia-associated AML1/ETO translocation in a subset of normal HSCs 
and mature blood cells during remission period (Miyamoto, Weissman, \& Akashi, 2000). Subsequent sequencing studies of normal HSC DNA from AML patients in remission consistently showed frequent occurrence of single driver mutation that possess premalignant potential (Corces-Zimmerman, Hong, Weissman, Medeiros, \& Majeti, 2014; Jan et al., 2012; Shlush et al., 2014). Remarkably, CH arises from mutations in a very small number of genes. In fact, two-thirds of $\mathrm{CH}$ mutations are found in the two enzyme-encoding genes: DNMT3A and TET2; both are involved in DNA methylation. The next most commonly mutated genes are $A S X L 1$ and splicing factors (SF3B1, SRSF2, PRPF8, U2AF1) (Jaiswal \& Ebert, 2019).

To differentiate non-malignant clonal hematopoiesis with cancer-associated mutations from other types of clonal hematopoiesis, the term clonal hematopoiesis of indeterminate potential (CHIP) was introduced (Steensma et al., 2015). In other words, an individual who has cancer-associated mutation in their blood cells in the absence of frank malignancy or other clonal entity is considered to carry CHIP. In addition, another criterion for CHIP is the threshold of variant allele fraction, which is set at $2 \%$ (or $4 \%$ of cells); this can be lowered when other prognostic factors are present (Jaiswal \& Ebert, 2019). CHIP is particularly significant in myeloid malignancies as mutations frequently found in CHIP are also driver mutations of myelodysplastic syndrome (MDS) (Bejar et al., 2011; Lindsley et al., 2017; Papaemmanuil et al., 2013), myeloproliferative disorders (Nangalia et al., 2013), acute myeloid leukemia (AML) (Cancer 
Genome Atlas Research et al., 2013), and certain lymphoid cancers (Couronne, Bastard, \& Bernard, 2012). It is of significant clinical implication to point out that the size of the mutant clone does matter; the larger the clone, the higher chance that it would develop into malignancy (Jaiswal et al., 2014).

Consistent with human studies, genetic models with loss-of-function mutations in either Dnmt3a or Tet2 showed HSCs with enhanced competitive repopulating potential and being more prone to leukemogenesis in the presence of additional mutations (Challen et al., 2011; Ko et al., 2011). In fact, age-associated alterations in hematopoiesis in mice have been well-characterized and continue to be an intense area of investigation (Broxmeyer et al., 2020; Busque, Buscarlet, Mollica, \& Levine, 2018; Wahlestedt \& Bryder, 2017). Generally, aged HSCs have been demonstrated to exhibit a decline in engraftment (Morrison, Wandycz, Akashi, Globerson, \& Weissman, 1996) (Rossi et al., 2005) (Sudo, Ema, Morita, \& Nakauchi, 2000) and homing (Liang, Van Zant, \& Szilvassy, 2005) capabilities with reduced lymphoid differentiation and concurrently myeloid-biased lineage output (Signer, Montecino-Rodriguez, Witte, McLaughlin, \& Dorshkind, 2007) (Miller \& Allman, 2005). It is also observed that there is an expanded number of phenotypically-defined HSCs in aged BM; however, whether their self-renewal capacity increases (Rossi et al., 2005) or decreases (Dykstra, Olthof, Schreuder, Ritsema, \& de Haan, 2011) still needs more investigation. 
Functional impairments of aged HSCs as compared to young HSCs when transplanted into young recipients suggest that the age-associated functional defects may be due to cell intrinsic factors. Such autonomous factors have been studied including changes in genetic and epigenetic landscapes, altered metabolic programs and cell polarity (Broxmeyer et al., 2020; Rossi et al., 2005). It is noteworthy to be aware that the collection process under ambient air (as most studies have been done) might confound the true characteristics of aged HSCs especially in vivo. Previous work has shown that exposure of HSCs to ambient oxygen levels within minutes significantly reduced their engrafting potential, a process termed extra physiological oxygen stress/shock or EPHOSS (Mantel et al., 2015). In fact, isolation and processing of aged HSCs in hypoxic conditions restored their functional properties to a significant level (M. L. Capitano, Mohamad, S. F., Cooper, S., Guo, B., Huang, X., Gunawan, A.M., Sampson, C., Ropa, J., Srour, E.F., Orschell, C.M., \& Broxmeyer, H.E., 2020). On the other hand, young HSCs transplanted into aged BM showed a reduction in engraftment as compared to young BM (Ergen, Boles, \& Goodell, 2012; Rossi et al., 2005), hence proposing an important role also of extrinsic factors in aged hematopoiesis. Interestingly, administration of thrombin-cleaved OPN (its secretion by BM stroma normally goes down with age) mitigated some functional defects of aged HSCs (Guidi et al., 2017). Notably, there is a shift in bone versus fat formation in aged BM MSCs with a tendency toward increasing adiposity; this could potentially dampen hematopoiesis (Ambrosi et al., 2017; Singh et al., 2016) (Naveiras et al., 2009) as well as promoting the myeloid skewing in lineage 
output (Adler, Green, Pagnotti, Chan, \& Rubin, 2014). With age, the BM vasculature is also altered with higher ROS levels, more leaky and lower concentrations of HSC supportive factors like CXCL12, SCF, and Jagged1. Coculture of aged HSCs with young endothelial cells can restore their repopulating potential, whereas co-culture of young HSCs with aged ECs impaired HSC function and promoted myeloid bias differentiation (Poulos et al., 2017). Future in-depth understanding of the aged BM niche may provide insights for early interventions that can potentially rescue or even prevent some of the functional impairments of HSCs, which are clinically relevant for both transplantation and malignancy fields.

\subsection{Leptin and leptin receptor}

\subsubsection{Discoveries of leptin/leptin receptor and their roles in non- hematopoietic systems}

Discovered in the early 1990 s as a key regulator of body weight and energy expenditure, leptin (LEP) has since been studied extensively in multidisciplinary fields (Bahary, Leibel, Joseph, \& Friedman, 1990; J. M. Friedman \& Halaas, 1998; J. M. Friedman, Leibel, Siegel, Walsh, \& Bahary, 1991; Tartaglia et al., 1995; Zhang et al., 1994). Different organs are known producers of LEP including adipose tissue, placenta, stomach, etc. Hence, it is not surprising that LEP, a 16kDa polypeptide hormone, exerts both central and peripheral effects by binding to its receptor (LEPR) (Campfield, Smith, Guisez, Devos, \& Burn, 1995; Y. Zhou \& Rui, 2013). LEPR exists in multiple isoforms as a result of alternative splicing; 
the long-isoform $(O b-R b)$, but not the short isoforms (Ob-Ra, Ob-Rc, Ob-Rd, Ob$R e)$, has been demonstrated to fully induce intracellular signaling mostly through the JAK/STAT pathway (Gorska et al., 2010; Lee et al., 1996). As a neuroendocrine hormone, the effects of LEP on feeding behaviors are mediated in part through activation of proopiomelanocortin neurons and inhibition of neuropeptide Y/agouti-related protein neurons in the hypothalamus (Cheung, Clifton, \& Steiner, 1997; Inui, 1999; Smith, Campfield, Moschera, Bailon, \& Burn, 1998; Thornton, Cheung, Clifton, \& Steiner, 1997). However, its central effects are more intriguingly complex, and involve roles in depression, stress response, dopaminergic reward circuits, reproduction and thermoregulation (Barb, Hausman, \& Lents, 2008; Chou \& Mantzoros, 2014; J. Friedman, 2016; J. M. Friedman, 2019; Schneider, 2004).

Being secreted mainly by adipocytes in proportion to body fat mass, LEP also acts as a proinflammatory adipokine. This is scientifically logical since immune functions are well-known to be tightly linked to nutritional status, and LEP is a critical messenger of the body energy storage throughout the entire life of an individual. It was reported early on that serum LEP levels and Lep mRNA expression increases following acute inflammation events or proinflammatory cytokine injection (Sarraf et al., 1997). In fact, the roles of LEP and its signaling in mature immune cells have been well-investigated. For instance, starvationinduced immunosuppression and attenuated T-cell immunity were alleviated by 
treatment with LEP (G. M. Lord et al., 1998). Consistent with this, human LEP acted as a negative regulator of regulatory T cells (De Rosa et al., 2007).

\subsubsection{Leptin/leptin receptor in hematopoiesis}

\subsubsection{Leptin/leptin receptor in adult non-malignant hematopoiesis, via microenvironmental stromal cells}

In the study of adult hematopoiesis under steady-state conditions, most work has focused on the roles of LEPR-expressing stromal cells in the BM niches. This is not a great surprise since adipocytes, a potential major source of BM LEP, are themselves a critical component of niche cells (Laharrague et al., 1998; Naveiras et al., 2009; B. O. Zhou et al., 2017). It is well-appreciated that HSCs are maintained, nurtured and regulated by an intricate and highly dynamic network of both cellular and molecular factors (Broxmeyer et al., 2012; Broxmeyer et al., 2007; Morrison \& Scadden, 2014; Sarkaria, Decker, \& Ding, 2018; Scadden, 2014). In order to delineate specific cellular sources of important growth factors for HSC homeostasis, a stem cell factor (Scf) green fluorescent protein (Gfp) knock-in murine model was employed to elegantly demonstrate that SCF from endothelial cells (ECs) and LEPR-expressing perivascular stromal cells were indispensable for BM HSC maintenance. In contrast, deletion of SCF from osteoblasts, Nestin-cre- or hematopoietic cells had insignificant effects on the BM HSC pool (Ding et al., 2012). Notably, MSCs (including LEPR-expressing stromal cells) expressed SCF at higher levels than endothelial cells (Asada et al., 2017). A follow-up study further demonstrated that SCF from endothelial cells was 
mainly critical for HSCs, whereas SCF from LEPR-expressing stromal cells was required not only by HSCs but also by a variety of progenitor cells including common lymphoid progenitors, common myeloid progenitors, megakaryocyteerythrocyte progenitors and other more differentiated precursor cells (Comazzetto et al., 2019).

To elucidate roles of niche cells, including LEPR ${ }^{+}$stromal cells, in context of hematopoietic recovery post-irradiation, a similar genetic approach as the above studies was utilized but to study the specific cellular source of pleiotrophin (PTN) rather than SCF in murine BM (Himburg et al., 2018). At baseline, using Ptn floxed/floxed mice (Ptn ${ }^{f l / f}$ mice) that were crossed with Cre-expressing mice to conditionally delete Ptn in different cell types, it was found that under homeostasis LEPR ${ }^{+}$stromal cells were an indispensable source of PTN required for HSC maintenance. In contrast, conditional deletion of Ptn from other cell types such as ECs, hematopoietic cells or osteoblasts did not affect HSC content nor HSC repopulating function in long-term competitive transplant assays. Interestingly, when mice were exposed to a sublethal dose of total body irradiation (500 cGy TBI), a shift in the roles of LEPR ${ }^{+}$stromal cells versus VEcad $^{+}$ECs in hematopoietic recovery occurred. Even though Ptn deletion from either LEPR ${ }^{+}$stromal cells or VE-cad ${ }^{+}$ECs resulted in reduction in numbers of lin`sca- $1^{+}$ckit $^{+}$(LSK; containing HSCs and progenitors) cells and myeloid progenitors by phenotyping analyses, only PTN from VE-cad ${ }^{+}$ECs was required for recovery of long-term (LT) -HSCs. BM cells from irradiated VE-cad-Cre;Ptn ${ }^{f|f t|}$ 
but not LepR-Cre;Ptn fl/fl mice exhibited reduced repopulating potential in both primary and secondary transplants compared to irradiated control mice. Secondary transplants allow one to assess the self-renewal capacity of HSCs.

Moreover, altered LEP signaling has been well-documented in obesity (Myers, Leibel, Seeley, \& Schwartz, 2010). Obesity is a risk factor for hematologic malignancies. Patients with obesity have increased mortality rate and HCTassociated complications. The indirect involvement of LEP signaling on hematopoietic output by modulating BM stromal cells in the context of exercise and chronic inflammation such as in atherosclerosis and cardiovascular diseases has been reported (Frodermann et al., 2019). Voluntary exercise induced $\mathrm{HSC} / \mathrm{HPC}$ quiescent-promoting niche factors only in LEPR ${ }^{+}$stromal cells, but not in other niche cellular components, while reducing adipose-tissue-derived LEP production. Also, LEP supplementation during exercise increased levels of circulating leukocytes, LSK cell proliferation and decreased gene expression of BM Angpt1, Cxcl12 and Vcam1 (Frodermann et al., 2019). These findings were not mediated through LEP signaling on HSCs/HPCs; effects of exercise remained unaffected in wild-type (WT) mice transplanted with $L e p R^{-/-} \mathrm{BM}$ from $d b / d b$ mice. $d b / d b$ (or Lep $R^{-/}$) mice carry a genetic mutation in the Lepr gene that causes LEPR inactivation (Bahary et al., 1990). The model is widely used for their metabolic phenotypes - obesity, hyperinsulinemia, hyperglycemia, etc. In contrast, cell-specific conditional deletion of $L e p R$ in stromal cells using Prrx1creER $^{T 2}$; Lepr ${ }^{f / f I}$ mice showed a reduction in LSK cell proliferation and circulating 
leukocytes while enhancing expression of HSC maintenance niche factors (Frodermann et al., 2019). This resembled the phenotype in exercising mice. Absence of LEP signaling in stromal cells of these mice alleviated the rise in LSK cell proliferation and systemic leukocytosis post-myocardial infarction (MI) (Frodermann et al., 2019). Treatment with LEP-neutralizing antibody 30 min after MI reduced BM colony-forming granulocyte-macrophage progenitor cell proliferation and monocytes in the blood and at the site of the infarct. This resulted in less inflammation and fibrosis and helped accelerate recovery. Similar results were observed in a different model of atherosclerosis (Frodermann et al., 2019) . These well-designed studies highlighted the significant roles that LEPRexpressing stromal cells play in both steady-state and emergency hematopoiesis. Even though LEP/LEPR involvement in potential various pathological conditions in the context of hematopoiesis is only in its infancy, these findings may have therapeutic potential in the future.

\subsubsection{Leptin/leptin receptor in hematopoietic stem and progenitor cells}

Not long after discoveries of LEP and its receptor, a novel sequence was cloned, namely B219, which was found in primitive hematopoietic cells isolated from the yolk sac, early fetal liver and some lymphohematopoietic cell lines. The sequence was expressed in at least four isoforms including one that resembled the recently published LEPR; its amino acid sequence shared similarity with granulocyte colony stimulating factor (G-CSF) receptor, gp130 and the leukemia inhibitory factor (LIF) receptor (Bahary et al., 1990; Cioffi et al., 1996). In that 
same year, cloning of different isoforms of $L E P R$ from human hematopoietic cell cDNA libraries were reported (Gainsford et al., 1996). Ectopic expression of the long isoform $O B-R b$ in murine $\mathrm{Ba} / \mathrm{F} 3$ and $\mathrm{M} 1$ cell lines respectively induced cell proliferation and differentiation. However, it was not clear whether LEP had effects on endogenously expressed LEPR and if so, in which hematopoietic cell populations. LEP enhanced cytokine production and phagocytosis by murine peritoneal macrophage (Gainsford et al., 1996). While these findings were limited to in vitro work, they spurred interest in more recent years in the study of LEP and its receptor in the field of hematology.

\subsubsection{Leptin/leptin receptor in hematologic malignancies}

There have been numerous studies in patients with both myeloid and lymphoid malignancies that looked at changes in plasma LEP levels during the course of the diseases and showed leukemic cells expressing leptin receptor isoforms (Gorska, Popko, \& Wasik, 2013; Konopleva et al., 1999; Moschovi et al., 2010; Mouzaki et al., 2009; Nakao et al., 1998; Ozturk, Avcu, \& Ural, 2012; Tsiotra et al., 2005; Wasik et al., 2006; Wex et al., 2002; M. Yilmaz et al., 2008). Unfortunately, the majority of these publications did not determine whether there were any distinct functional properties of LEPR-expressing leukemic cells or if LEP signaling played a role in human leukemia pathogenesis and maintenance, and if so, how. Still, some of the work provided proof-of-principle evidence of LEP and its receptor being possibly involved to some extent in different types of 
leukemias using patient-derived samples, murine models and human cell, mouse recipient xenograft studies.

As early as 1999 , using reverse-transcriptase polymerase chain reaction to study gene expression levels, it was demonstrated that approximately $50 \%$ of collected patient specimens of either newly diagnosed primary or secondary acute myeloid leukemia (AML) expressed higher levels of the long isoform, than the short isoform, of $L E P R$. This was also true for primary and recurrent acute promyelocytic leukemia. In contrast, only the short isoform could be found in healthy human promyelocytes (defined as $\mathrm{CD} 34^{-} \mathrm{CD} 33^{+}$or $\mathrm{CD} 34^{-} \mathrm{CD} 13^{+}$). LEPR was not detected in chronic or acute lymphocytic leukemic cells (Konopleva et al., 1999). Patient-derived myeloid leukemic blasts and leukemic cell lines proliferated at low levels when treated with human recombinant LEP alone and significantly at higher levels in the presence of interleukin-3 (IL-3), G-CSF, or SCF (Konopleva et al., 1999).

Similarly, another paper characterized in vitro effects of LEP on cultured human AML blasts and compared systemic LEP levels in AML patients with healthy controls (Bruserud, Huang, Glenjen, Gjertsen, \& Foss, 2002). Serum LEP concentrations were significantly lower in untreated AML patients, but were not significantly different for patients in remission compared to normal subjects. Whether or not this change in serum LEP levels might have been related to the treatments was not further investigated. The authors (Bruserud et al., 2002) then 
looked at in vitro responses of patient-derived AML blasts (mixed CD $34^{+}$and CD34- leukemic cells) to LEP treatment and found that LEP significantly increased blast cell proliferation and secretion of proinflammatory cytokines including IL-1 $\beta$, IL-6, tumor necrosis factor alpha (TNFa) and granulocytemacrophage colony-stimulating factor (GM-CSF) in a subset of patients. Notably, effects of LEP were observed at supraphysiological levels $(2 \mathrm{ug} / \mathrm{mL})$, so it is still unclear how these blasts might have behaved differently in response to physiological levels of leptin in vivo. Plus, systemic LEP concentrations may significantly differ from local sources such as in the leukemic BM niche. This was followed up with colony-forming unit assays (used to detect HPCs) using the same LEP concentration of $2 \mathrm{ug} / \mathrm{mL}$ in two different settings. Specifically, AML blasts were cultured for 7 days with or without LEP before being washed and plated, or the cells were plated directly in the presence or absence of LEP with GM-CSF and Erythropoietin. LEP was found to have minimal effects on colony formation, blast differentiation and apoptotic activity. However, results varied among patient samples. Overall, while these two studies were of some interest and suggested a potential new therapeutic target involving LEPR signaling in AML, the scope was limited to in vitro work, and the sizes of patient samples were relatively small. Thus, more in-depth future work with rigorous in vivo functional assessments and mechanistic insights are warranted. What is known regarding LEP/LEPR in hematopoiesis is outlined in Table 1.1. 


\begin{tabular}{|c|c|}
\hline \multicolumn{2}{|c|}{ Table 1.1 LEP/LEPR in hematopoiesis. What is known? } \\
\hline $\begin{array}{l}\text { LEP/LEPR in } \\
\text { BM } \\
\text { mesenchymal } \\
\text { stem/stromal } \\
\text { cells (MSCs) }\end{array}$ & $\begin{array}{l}\text { - } \text { LEPR }^{+} \text {BM MSCs were an indispensable source } \\
\text { of SCF (Asada et al., 2017; Ding et al., 2012) and } \\
\text { PTN (Himburg et al., 2018) that were required to } \\
\text { maintain HSC homeostasis . } \\
\text { - SCF derived from LEPR+ BM MSCs was critical } \\
\text { for both HSCs and progenitor cells under steady } \\
\text { state (Comazzetto et al., 2019). } \\
\text { Exercise induced expression of niche factors only } \\
\text { in LEPR }{ }^{+} \text {BM MSCs while leptin supplementation } \\
\text { reversed this effect (Frodermann et al., 2019). }\end{array}$ \\
\hline $\begin{array}{l}\text { LEPR/LEPR } \\
\text { in HSC/HPCs }\end{array}$ & $\begin{array}{l}\text { - Isoforms of LEPR were found in both murine and } \\
\text { human primitive hematopoietic cells (Cioffi et al., } \\
\text { 1996; Trinh T, 2020). } \\
\text { - Lep signaling through LEPR+ BM MSCs induced } \\
\text { LSK proliferation and hematopoietic output in } \\
\text { post-myocardial infarction and atherosclerosis } \\
\text { (Frodermann et al., 2019). } \\
\text { - LEPR+LSK cells were highly enriched for } \\
\text { functional self-renewing LT-HSCs, and } \\
\text { LEPR+HSCs represented a subset of more }\end{array}$ \\
\hline
\end{tabular}




\begin{tabular}{|c|c|c|}
\hline & & $\begin{array}{l}\text { robustly repopulating LT-HSCs as compared to } \\
\text { LEPR-HSCs (Trinh T, 2020). }\end{array}$ \\
\hline $\begin{array}{l}\text { LEP/LEPR in } \\
\text { hematologic } \\
\text { malignancies }\end{array}$ & $\bullet$ & $\begin{array}{l}\text { A significant proportion of patient-derived } \\
\text { leukemia samples expressed isoforms of Lepr } \\
\text { (Gorska et al., 2013; Konopleva et al., 1999; } \\
\text { Mouzaki et al., 2009; Nakao et al., 1998; Ozturk et } \\
\text { al., 2012; Tsiotra et al., 2005; Wasik et al., 2006; } \\
\text { Wex et al., 2002). } \\
\text { LEP induced myeloid leukemic blasts and cell } \\
\text { lines to proliferate at low level while enhancing } \\
\text { effects of IL-3, G-CSF or SCF (Konopleva et al., } \\
\text { 1999). } \\
\text { At supraphysiological concentration, LEP } \\
\text { increased patient-derived AML blast proliferation } \\
\text { and secretion of IL-6, TNFa, IL-1ß and GM-CSF } \\
\text { (Bruserud et al., 2002). } \\
\text { LEPR signaling mediated the anti-leukemic } \\
\text { protective effects of fasting in B-ALL and T-ALL } \\
\text { but not AML models by promoting blast cell } \\
\text { differentiation (Z. Lu et al., 2017). }\end{array}$ \\
\hline
\end{tabular}




\begin{tabular}{|l|l|}
\hline & $\begin{array}{l}\mathrm{LEPR}^{+} \text {MSC alterations (e.g. lower expression of } \\
\text { niche factors, upregulating genes involved in } \\
\text { fibrosis) played a key role in the pathogenesis of } \\
\text { PMF (Decker et al., 2017). }\end{array}$ \\
\hline
\end{tabular}




\subsubsection{Leptin/leptin receptor in other stem cells}

The therapeutic value of LEP/LEPR in different stem cell-based systems has been explored over decades. Here, we describe some of the recent work that provided evidence of how LEP and its receptor regulate stem cell functions, with some showing potential clinical applications.

Thus far, most of the studies on LEP/LEPR in stem cell biology under homeostasis have not fully addressed mechanistically whether and how LEP/LEPR directly regulates stem cell functions. It was recently reported using mass spectrometry-based global proteomic analyses that $L e p R^{-/}$(or $\left.d b / d b\right)$ mouse embryonic fibroblasts had increased levels of phosphorylated ERK1/2, RAPTOR and MEK1/2 with the most differentially regulated pathways involving mitochondrial dysfunction and oxidative phosphorylation (M. K. Gupta et al., 2020). Likewise, induced pluripotent stem cells (iPSCs) derived from $d b / d b$ mice, and hence carrying the Lepr mutation, exhibited significant lowered protein levels of the pluripotency markers OCT4 and NANOG. $d b / d b$ iPSCs also revealed a proteomic profile that involved pathways regulating mitochondrial functions, protein synthesis (including upregulation of eukaryotic translation initiation factor 4e (EIF4E) and EIF4G) and pluripotency (M. K. Gupta et al., 2020). Molecularly, $d b / d b$ iPSCs were demonstrated to have a reduction in STAT3 phosphorylation in response to LEP treatment even though at baseline there was no difference as compared to control iPSCs. Chromatin immunoprecipitation analysis revealed enrichment of Stat3 binding regions on the promoter of Eif4e of $d b / d b$ iPSCs, 
hence leading to enhanced protein synthesis as shown by a higher polysome/monosome ratio in polysome profiling study and an increased basal oxygen consumption rate in metabolic profiling study. Overall, these findings suggest a role(s) of LEP/LEPR both in embryonic stem cell development and in regulating pluripotency-associated genes.

In a highly mechanistic study it was demonstrated that production of LEP induced by hypoxia preconditioning modulated the level of mitochondrial fusion protein OPA1, and hence improved human MSC survival and its therapeutic effects under ischemic conditions (F. Yang et al., 2018). While the study showed that overexpression of LEP in human MSCs promoted their in vivo therapeutic effects including enhanced cardiac function and increased angiogenesis in cardiac infarction, it was unclear whether endogenous circulating LEP played any role, and if so, would endogenous LEP also act mainly through the GSK3/OMA1/OPA1 pathway? In a follow up study (F. Yang et al., 2019), it was shown that LEP increased glycolysis in MSCs by upregulating the glucose transporter sodium-glucose symporter 1 (SGLT1), and OPA1 was required for the effect of leptin on glycolysis. However, it was not well-addressed how OPA1 could be involved in the enhanced expression of SGLT1 (F. Yang et al., 2019). Notably, LEP has been well-known for its angiogenic effect, and was shown to increase endothelial cell differentiation from murine embryonic stem cells (Kurtovic et al., 2015; Sierra-Honigmann et al., 1998). Furthermore, obese patients who have increased risk for cardiovascular diseases may be more likely 
to have higher systemic LEP levels and LEP resistance. Considering that this could also have a negative impact in vivo (Frodermann et al., 2019), it is important to weigh potential risks versus benefits as to how the cells may be preconditioned before transplantation.

On the other hand, LEP signaling has been studied extensively in context of solid tumor cancer stem cells (CSCs), particularly in breast cancer stem cells (BCSCs) (Crean-Tate \& Reizes, 2018; Lipsey, Harbuzariu, Daley-Brown, \& GonzalezPerez, 2016). CSCs represent one of the key challenges to cancer treatments; hence, understanding how CSCs are initiated and maintained by the tumor microenvironment will have an impact on development of more effective therapies. It has been demonstrated that LEPRb maintained stem-cell-like characteristics of triple negative breast cancer cells (TNBC) by inducing expression of stem cell self-renewal transcription factors OCT4, SOX2 and NANOG (Zheng et al., 2013). Using a NANOG promoter-driven GFP activity readout, it was observed that mouse LEPR when introduced into human TNBC MDA-MB-231 and HCC70 non-CSCs induced NANOG expression (Thiagarajan et al., 2017). Two other markers of CSCs SOX2 and OCT4 were also expressed at higher levels in LEPR-expressing non-CSCs as compared to control nonCSCs; this suggested that overexpression of LEPRb was sufficient to convert non-CSCs into CSCs. Still, it was not clear whether in situ non-CSCs can naturally acquire LEPRb expression to become CSCs and if the transition is reversible or not. While LEP signaling can have multiple downstream effectors, 
the authors showed that LEPRb specifically activated Tyr1077 and Tyr1138 phosphorylation, which in turn transactivated JAK2/STAT3, a major signaling pathway of LEPRb activation.

In a more mechanistic study, it was demonstrated that the pan-JAK inhibitor AZD1480 decreased viability of HCC1937 CSCs as compared to its non-CSC counterpart and suppressed tumorsphere formation from MCF7 CSCs (T. Wang et al., 2018). To understand molecular pathways that governed this phenomenon, they performed RNAseq analyses on AZD1480-treated MCF7 tumorsphere cells and discovered that genes involved in lipid metabolism were downregulated (T. Wang et al., 2018). Mechanistically, STAT3 modulated expression of genes involved in fatty acid oxidation (FAO) including the ratelimiting enzyme carnitinepalmitoyl transferase 1 (CPT1), which was required to maintain tumor cells in a stem-cell-like state. In line with this, inhibition of STAT3 in BCSCs reduced their self-renewing capacity. To further explore which upstream regulator affects STAT3/FAO pathway in BCSCs, they performed an adipokine array using breast adipocyte-conditioned medium. Among the most abundantly expressed adipokines was LEP. By using quantitative real time polymerase chain reaction and flow cytometry analyses, the only adipokine receptor detected at increased levels in the patient-derived BBM2 and BBM3 tumorsphere cells was LEPR. Consistent with this finding and the fact that LEPR signaling is known to activate JAK2/STAT3 pathway, further experiments revealed that adding LEP to culture media increased tumorsphere formation, as 
well as phosphorylation of JAK2 and STAT3. Likewise, treatment with LEP neutralizing antibody reversed the increase in expression of $C P T 1 B$ and $A C A D M$, both enzymes involved in the FAO pathway, and reduced the percentage of BCSCs in the breast tumors. In fact, in patient tumor samples it was found that chemoresistant metastatic breast tumor sections expressed higher level of LEPR with LEPR-positive cells having increased CPT1B expression. Altogether, these in-depth mechanistic studies using patient-derived samples strongly suggested that leptin receptor and its downstream effectors JAK2/STAT3/CPT1 may be potential drug targets that will specifically eradicate the chemoresistant BCSCs. 


\section{CHAPTER TWO: Materials and Methods}

\subsection{Mice}

\subsubsection{Mouse strains}

Female and male Boy/J, B6xBoy/J F1 (herein referred to as $F_{1}$ ), and C57BL6/J (8 - 12 weeks old) were received from the In Vivo Therapeutics Core, an on-site breeding core facility at Indiana University School of Medicine. For studies using $\operatorname{Lep}^{-/}(\mathrm{ob} / \mathrm{ob})$ or Lepr ${ }^{--}(\mathrm{db} / \mathrm{db})$ mouse models, the mice (male or female) were purchased from Jackson Laboratory and acclimated in our animal facility for at least one week before the experiment dates. These mice arrived at around $6-8$ weeks old and were used at around $2.5-3$ months old. For all experiments, mice were age- and sex-matched. Animals were maintained under light- and temperature-controlled conditions (12-hour light/12-hour dark cycle, $21-24^{\circ} \mathrm{C}$ ) and were housed in groups of 5 maximum per cage according to age, sex, and genotype.

\subsubsection{Mouse irradiation for transplantations}

F1 recipient mice were irradiated 24 hours prior to transplantation using split doses - $700 \mathrm{cG}$ followed with $400 \mathrm{cGy}$ (at least 3 hours apart) in primary transplants and single dose $-950 \mathrm{cG}$ in secondary transplants. 


\subsection{Preparation of cells from fresh BM}

Bone marrow were flushed from femurs using ice-cold MACS buffer (PBS supplemented with $0.5 \% \mathrm{BSA}$ and $2 \mathrm{mM}$ EDTA) and dissociated into single cell suspension by pipetting with Eppendorf Combitips (Eppendorf). As exceptions, for limiting dilution assay of LEPR+LSK and LEPR-LSK cells and RNA-seq sample preparation, BM cells were harvested by crushing spines, pelvis, femurs and tibias in ice-cold MACS buffer.

\subsection{Enrichment of mouse Lin-BM cells and human (h) CB CD $34^{+}$cells} Mouse BM cells were flushed from the femurs (in some cases also tibias and pelvis) of C57BL6/J into ice-cold MACS buffer. Mouse BM lineage depletion was done following manufacturer's protocol of the direct mouse lineage cell depletion kit (Miltenyi Biotec). Human umbilical CB units were either purchased from Cord Use Cord Blood Bank (Orlando, Florida, USA) or obtained as a gift from Cleveland CB Bank and used within 24 to 48 hours post-collection. In detail, CB was washed in PBS at a volume ratio of 1:1 before Ficoll gradient separation using Ficoll-Paque PLUS (GE Healthcare Bio-Sciences AB) for mononuclear cells. $\mathrm{CB}$ CD34 ${ }^{+}$cells were then isolated using the CD34 MicroBead Kit (Miltenyi Biotec) following manufacturer's protocol.

\subsection{Cell staining for flow cytometry analyses and sorting}

For BM analyses at the last time-points of transplants, cells were counted and stained at a concentration of $2.5-4 \times 10^{6}$ cells in $200 \mu$ MACS buffer per tube on 
ice for 20 minutes and washed in PBS. For staining of Lepr HSCs and HPCs, cells were first incubated with fluorescently conjugated primary anti-mouse antibodies ( $0.2 \mu \mathrm{g}$ of antibody was used per one million cells) and anti-LEPRbiotin antibody (R\&D systems, BAF497, 0.2 ug per one million cells), washed in PBS, then incubated with secondary streptavidin-PE/Cy7 (Biolegend, Cat.\# 405206) for another 20 minutes on ice (1:500) and washed in PBS. Cells were resuspended in diluted DAPI solution (BD Biosciences, 1:10000) before being analyzed on a LSR II flow cytometer (BD Biosciences) or sorted using a FACSAria or SORP Aria flow cytometers (BD Biosciences). For other HSC and HPC staining panels, cells were fixed in $1 \%$ paraformaldehyde and analyzed the next day. Data analysis was done using FlowJo 10.7.2 software (BD). Singlecolor compensation was included for each experiment; gating strategies were determined with fluorescence minus one controls. Percent of each population was used to calculate absolute numbers per femur. For human CB phenotyping, CD34+-enriched cells were first isolated using the CD34 MicroBead Kit (Miltenyi Biotec) following manufacturer's protocol. Staining procedure was performed in a similar fashion to mouse BM cells but with fewer cells per tube (about 200,000 400,000 cells). Full details on the antibodies and clones used for FACS analyses can be found in Table 2.1. 
Table 2.1 List of fluorophore conjugated antibodies used in the study

\begin{tabular}{|l|l|l|}
\hline \multicolumn{2}{|c|}{ Antibody } & \multicolumn{2}{|c|}{ Cluorophore } \\
\hline \multicolumn{2}{|c|}{ Mouse antibodies } \\
(CD3e, CD11b, \\
CD45R/B220, Ly-76, Ly- \\
6G and Ly-6C)
\end{tabular}




\begin{tabular}{|l|l|l|}
\hline CD34 & PE & MEC14.7 \\
\hline CD16/32 & PerCP/Cyanine5.5 & 93 \\
\hline CD127 & BV421 & A7R34 \\
\hline CD45.1 & FITC & A20 \\
\hline CD45.2 & APC & 104 \\
\hline CD11b & PE/Cy7 & M1/70 \\
\hline Ly-6G/Ly-6C (or Gr-1) & PE & RB6-8C5 \\
\hline CD3 & BV421 & 17 A2 \\
\hline CD45R/B220 & PerCP/Cyanine5.5. & RA3-6B2 \\
\hline Ki67 & PE & 16 A8 \\
\hline & \multicolumn{2}{|c|}{ Human antibodies } \\
\hline CD34 & PE & 581 \\
\hline CD38 & FITC & HIT2 \\
\hline CD45RA & PECF594 & HI100 \\
\hline CD90 & Pe-Cy7 & 52263 \\
\hline CD295 (LEPR) & Alexa Fluor 647 & \\
\hline
\end{tabular}




\subsection{Cell cycling assay}

For phenotypic cell cycling studies, freshly isolated BM cells were first partially depleted for Lin` cells, stained for surface markers on ice, fixed and permealized using BD Fixation/Permealization Solution Kit for $30 \mathrm{~min}$. Then they were stained with Ki67 for half an hour at RT (1:20 dilution, $200 \mathrm{uL} /$ million), washed once before being stained with DAPI (1:10,000 dilution, $500 \mathrm{ul} / \mathrm{million})$ for $20 \mathrm{~min}$ at RT. Cells were washed again and resuspended in PBS before being analyzed (Galvin et al., 2019; Pietras et al., 2014).

\subsection{Colony-forming unit assays}

Freshly sorted cells were plated at 200 cells $/ \mathrm{ml}$ in $1 \%$ methylcellulose culture medium supplemented with 30\% FBS (Fisher Scientific), 2 mM L-Glutamine (Lonza), $0.1 \mathrm{mM}$ hemin (Sigma-Aldrich), $50 \mathrm{ng} / \mathrm{ml} \mathrm{rmSCF} \mathrm{(R \& D} \mathrm{Systems),} 1 \mathrm{U} / \mathrm{ml}$ recombinant human erythropoietin (rhEPO, Amgen), and 5\% vol/vol pokeweed mitogen mouse spleen cell conditioned medium. Cells were cultured at $5 \% \mathrm{CO}_{2}$, $5 \% \mathrm{O}_{2}$ in a humidified incubator, and colonies were scored on day 6 based on morphologies of functional CFU-granulocyte, macrophage (CFU-GM), burstforming unit-erythroid (BFU-E), and CFU-granulocyte, erythrocyte, macrophage, megakaryocyte (CFU-GEMM) progenitors.

\subsubsection{High specific activity tritiated thymidine kill assay}

For high specific activity tritiated thymidine kill assay to determine \% CFU in Sphase of the cell cycle, cells were incubated in IMDM media containing either 
control diluent or $50 \mathrm{mCi}$ high specific activity tritiated thymidine for 30 minutes in the same incubator for cell culture aforementioned before washing and plating. For hCB CFU assay, cells were plated in $1 \%$ methylcellulose culture medium supplemented with 30\% FBS, 2 mM L-Glutamine, human recombinant EPO (1U/ml), SCF (50 ng/ml), GM-CSF (10 ng/ml) and IL-3 $(10 \mathrm{ng} / \mathrm{ml})$ and cultured under the same conditions as for mouse cells. Human CFU-GM and CFU-GEMM colonies were scored on day 14 after culture, as this combination of growth factors does not pick up BFU-E (Broxmeyer et al., 2012; Mantel et al., 2015).

\subsection{Competitive transplantation assays to assess engraftment and competitive fitness of HSCs}

Freshly sorted LEPR ${ }^{+}$LSK and LEPR-LSK (or LEPR ${ }^{+} H S C$ and LEPR-HSC) cells from pooled BM of donor C57BL6/J (CD45.1-CD45.2+) mice were intravenously (i.v.) injected with 100,000 unseparated BM cells of competitor BoyJ $\left(\mathrm{CD} 45.1^{+} \mathrm{CD} 45.2^{-}\right)$mice into lethally irradiated $\mathrm{F} 1$ recipient $\left(\mathrm{CD} 45.1^{+} \mathrm{CD} 45.2^{+}\right)$ mice $(n=5-7)$. At the last time point of primary transplant, marrow from primary engrafted mice was pooled within each group and transplanted i.v. into secondary recipient $\left(2 \times 10^{6}\right.$ cells per mouse, $\left.n=5-10\right)$ to assess the selfrenewal capacity of the HSCs. Percentages of donor CD45.1-CD45.2 ${ }^{+}$chimerism were determined in the PB at month (mo) 1, 2, 4 (and 5, 6, 7 in some cases) and BM at the last time point (Broxmeyer et al., 2012). 


\subsubsection{Limiting dilution assays (LDAs) to calculate numbers of functional HSCs}

It is important to note that phenotyping analyses using FACS only allows one to assess the numbers of HSCs present but do not provide any information about the functions of the cells. Hence, there is a need for LDAs to determine the numbers of functional HSCs.

\subsubsection{Competitive repopulating units (CRUs), measures of mouse BM functional HSCs}

Increasing doses of freshly sorted LEPR ${ }^{+}$LSK and LEPR-LSK cells $(50,100,150$, 250 cells) or LEPR+HSC and LEPR-HSC $(50,100,200)$ from pooled BM of donor C57BL6/J (CD45.1-CD45.2+ $)$ mice were i.v. injected with 100,000 unseparated BM cells of competitor BoyJ $\left(C D 45.1^{+} \mathrm{CD} 45.2^{-}\right)$mice into $\mathrm{F} 1$ recipient $\left(\mathrm{CD} 45.1^{+} \mathrm{CD} 45.2^{+}\right)$mice $(\mathrm{n}=5-7)$ that had been lethally irradiated $(700 \mathrm{cGy}$ followed with $400 \mathrm{cGy}$ ) 24 hours prior to transplantation. Donor chimerism $\left(\% \mathrm{CD} 45.1^{-} \mathrm{CD} 45.2^{+}\right)$was assessed in PB month 1, 2, 4 (and 5, 6, 7 in some experiments) and BM last time point. The number of mice with $1 \%$ (for LEPR+LSK vs. LEPR-LSK transplant) or $10 \%$ (for LEPR+HSCs vs. LEPR-HSCs transplant) or greater of donor-derived BM CD45.1-CD45.2+ was determined for each dose. The frequency of CRUs was calculated and plotted using ELDA software (http://bioinf.wehi.edu.au/software/elda/) (M. L. Capitano et al., 2019). 


\subsubsection{SCID-repopulating cells (SRCs), measures of human CB functional}

HSCs

For hCB LDA transplant, different doses $(1000,2500,5000)$ of freshly sorted total $\mathrm{CD} 34^{+}, \mathrm{LEPR}^{+} \mathrm{CD} 34^{+}$or LEPR-CD34 ${ }^{+}$cells were i.v. transplanted into young adult NSG mouse recipients $(n=5)$ that had been sublethally-irradiated (350 cGy) 24 hours prior to injection. Human $\mathrm{CD}_{4} 5^{+}$chimerism was determined at month 2, 4 in PB and month 4 in BM. The number of mice with $0.2 \%$ or greater of $\mathrm{hCD} 45^{+}$chimerism was assessed for each dose. The frequency of SRCs was calculated and plotted using ELDA software (http://bioinf.wehi.edu.au/software/elda/) (Chen et al., 2019; Mantel et al., 2015).

\subsection{Homing assay}

HSCs do not home to the BM after infusion into mice with absolute efficiency, hence the need to assess the homing efficiency of cells injected. Freshly sorted LEPR+LSK and LEPR-LSK cells (7000 cells) from pooled BM of donor C57BL6/J $\left(\mathrm{CD} 45.1^{-} \mathrm{CD} 45.2^{+}\right)$mice were i.v. injected into lethally irradiated $(700 \mathrm{cGy}$ followed with $400 \mathrm{cG}$, 24 hours prior to transplantation) BoyJ recipient $\left(C D 45.1^{+} C D 45.2^{-}\right)$mice. Seventeen hours post-injection, BM from host mice was harvested and analyzed by flow cytometry to determine the numbers of CD45.1CD45.2+ LSK CD150+, LSK and LK cells. 


\subsection{Bulk RNA-sequencing}

\subsubsection{Preparation of cells for RNA extraction}

Mouse BM cells were collected by crushing spines, pelvis, femurs and tibias from two young adult C57BL6/J mice into ice cold MACS buffer. Single cells were staining and sorted fresh on the same day. LEPR+ or LEPR- HSCs or MPPs were sorted directly into lysis buffer containing 2-betamercapthanol.

\subsubsection{Library preparation, data collection and analyses}

RNA was extracted right after sorting and subjected to library prep using Takara Smart-Seq V4 Ultra Low Input RNA kit. Libraries were sequenced using an Illumina NovaSeq 6000. Reads were trimmed by Cutadapt, and FastQC was used to check the quality of reads. Reads were then aligned and assigned to genes using STAR aligner and HTSeq feature counter. Differential gene expression analyses were performed using DESeq2. Gene set enrichment analyses were performed using the R package 'fgsea'.

\subsection{Statistical analyses}

Results are expressed as mean values \pm SD. A p value less than 0.05 was considered statistically significant. Mann-Whitney test or unpaired two-tailed Student's t test was used where indicated. Ordinary one-way ANOVA with posthoc Tukey's multiple-comparisons test was used when comparing 3 or more groups. Ordinary two-way ANOVA with post-hoc Tukey's multiple-comparison test was used when comparing groups of two different categorical variables. For 
limiting dilution assay transplants, Poisson statistical analysis was used.

Graphpad Prism outlier calculator (alpha $=0.05$ ) was used to exclude outlier data points (https://www.graphpad.com/quickcalcs/grubbs1/). 


\begin{abstract}
CHAPTER THREE: LEP/LEPR signaling is not required for HSC/HPC maintenance under homeostasis, but LEPR-expressing HSCs represent a subset of highly engrafting long-term functional hematopoietic stem cells
\end{abstract}

\title{
3.1 LEPR ${ }^{+}$cells are significantly outnumbered by LEPR' cells in young adult C57BL6/J WT mouse BM
}

Early studies by others suggested that leptin enhanced HPC colony formation (Bennett et al., 1996; Cioffi et al., 1996; Claycombe, King, \& Fraker, 2008; Gainsford et al., 1996; Umemoto et al., 1997). Furthermore, LEPR ${ }^{+}$stromal cells have been well-characterized as a critical component of BM niche cells for their role in the secretion of growth factors (GFs) to support HSCs and HPCs under steady-state and in regenerative conditions (Comazzetto et al., 2019; Ding et al., 2012; Himburg et al., 2018; B. O. Zhou et al., 2017; B. O. Zhou et al., 2014). Even though it was observed that hematopoietic cells only expressed the truncated short isoforms of Lepr (B. O. Zhou et al., 2014), I questioned whether germ-line knockout of Lep or Lepr might have an indirect effect on hematopoiesis since LEP/LEPR played such an important role in BM stromal cells. Towards this aim, I performed extensive phenotypic analyses on BM freshly collected from either WT mice or from Lep-/- or Lepr/- mice.

To confirm expression of LEPR on hematopoietic cells and determine percentages of LEPR-expressing cells among various HSC and HPC populations, flow cytometry analyses were utilized. I found that the majority of 
HSCs/HPCs did not express Lepr, consistent with reports that only $0.3 \%$ of BM cells are LEPR ${ }^{+}$cells (B. O. Zhou et al., 2014). Results were similar regardless of which marker panels was used. Using the CD34 and FLT3 markers, I found that LT-HSC (defined as LSKCD34-FIt3-) contained a significantly higher fraction of $\mathrm{LEPR}^{+}$cells compared to different progenitor populations including Common Myeloid Progenitor (CMP, defined as LKFcgR $\left.{ }^{\text {int }} \mathrm{CD} 34^{+}\right)$, GranulocyteMacrophage Progenitor (GMP, defined as $\mathrm{LKFcgR}^{\text {hi }} \mathrm{CD} 34^{+}$), MegakaryocyteErythroid Progenitor (MEP, defined as LKFcgR-CD34 ${ }^{+/}$) and Common Lymphoid

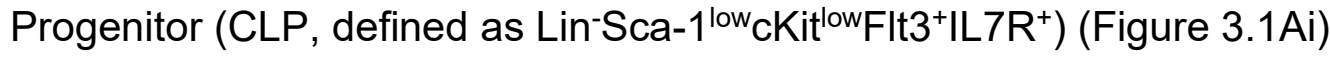
(Doulatov, Notta, Laurenti, \& Dick, 2012).

On the other hand, using the SLAM marker system with CD150 and CD48 as well as FLT3, it was found that the percentage of LEPR ${ }^{+}$cells in the long-term SLAM HSC (Lin-Sca- $1^{+}{ }^{+}{ }^{-K}$ it $^{+}$FIt3-CD150+CD48-) (LSK FIt3-CD150+CD48-, hereafter denoted as SLAM-HSC ${ }^{L T}$ ) population was significantly higher than that of short-term (ST) SLAM HSC (LSK FIt3-CD150-CD48- or SLAM-HSC ${ }^{\text {ST }}$ ) and different subsets of lineage-biased MPPs including the myeloid-biased MPP2 (LSK Flt3-CD150+CD48 ${ }^{+}$), the myeloid-biased MPP3 (LSK Flt3-CD150-CD48+), and the lymphoid-biased MPP4 (LSK Flt3 ${ }^{+}$CD150-CD48 ${ }^{+}$) (Figure 3.1Aii) (Pietras et al., 2015). 
Ai.

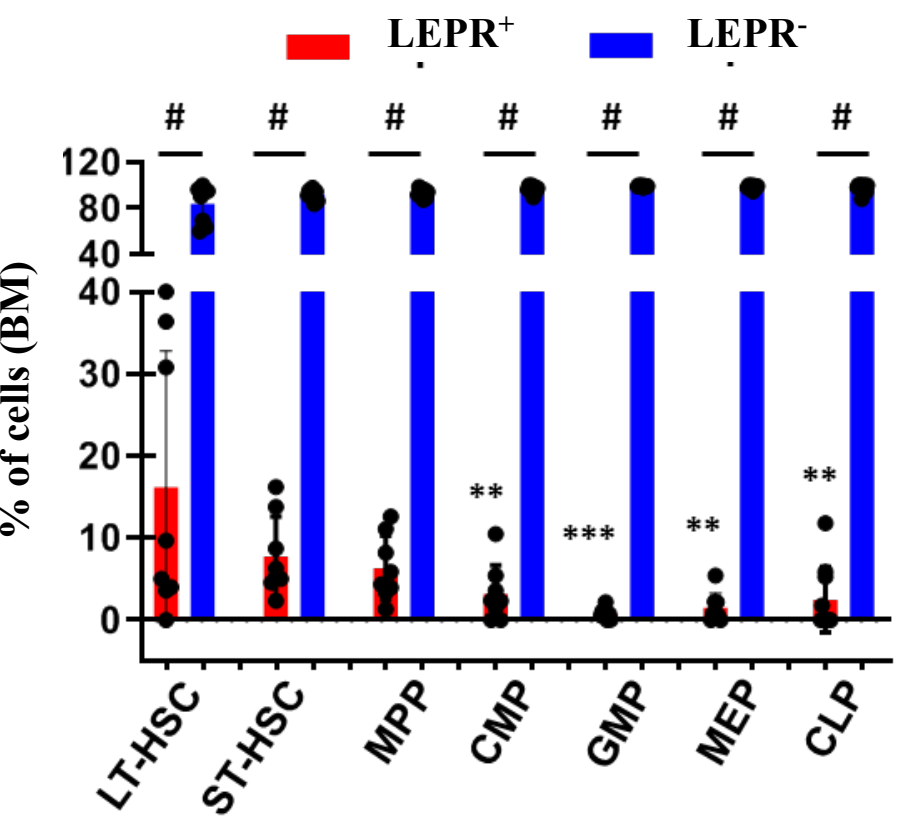

Aii.

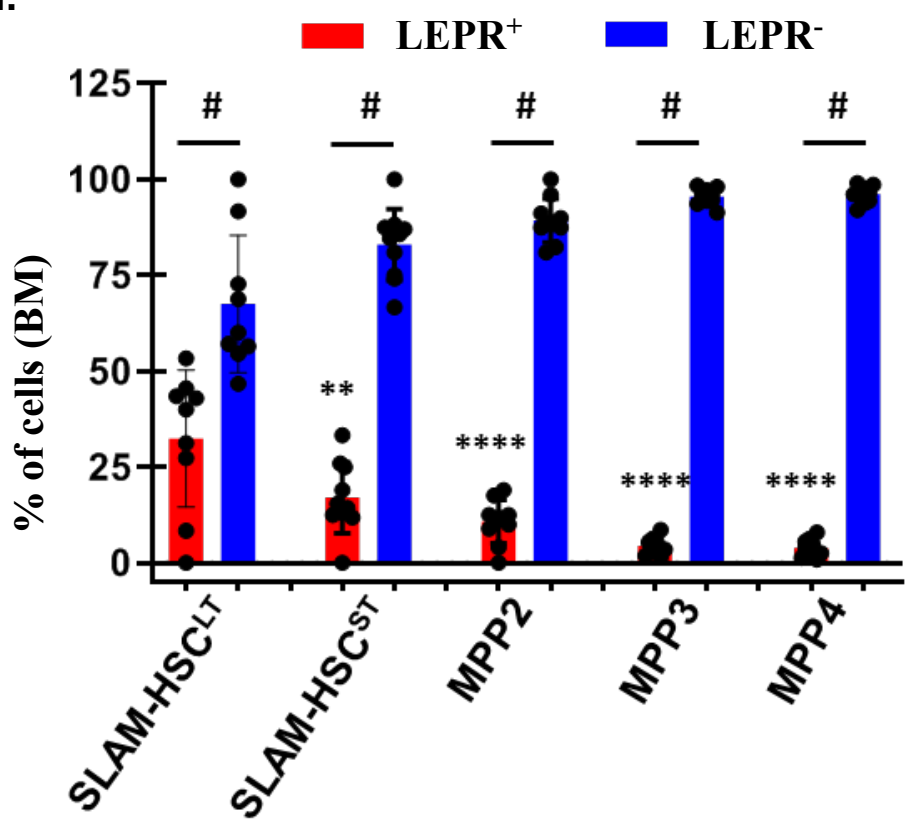


Figure 3.1_BM LEPR ${ }^{+}$cells are significantly outnumbered by LEPR' cells FACS analyses of freshly isolated BM cells from young adult C57BL/6J mice $(n=8-10)$.

(Ai). Percentages of LEPR ${ }^{+}$versus LEPR' cells (using the CD34 and FLT3 markers) within each population (LT-HSC, ST-HSC, MPP, CMP, GMP, MEP, CLP).

(Aii). Percentages of LEPR ${ }^{+}$versus LEPR ${ }^{-}$cells (using the SLAM markers CD150 and CD48 as well FLT3) within each population (SLAM-HSC ${ }^{L T}$, SLAM-HSC ${ }^{\text {ST }}$, MPP2, MPP3, MPP4).

All data are mean $\pm S D$. \# $p<0.0001$ using Mann-Whitney test comparing LEPR+ versus LEPR- cells within each population in (Ai) and (Aii), * $p<0.05,{ }^{* *} p<0.01$, ${ }^{* * *} p<0.001,{ }^{* * *} p<0.0001$ using Ordinary One-way ANOVA followed with posthoc Tukey's multiple comparison test to compare fractions of LEPR+ among different populations to SLAM-HSCLT in (Ai) or LT-HSC in (Aii). $\mathrm{N}=2$ (independently repeated experiments). 


\subsection{Germline Knockout of murine Lep or Lepr did not alter numbers of BM phenotypic HSCs/HPCs under homeostasis}

To determine whether LEP/LEPR signaling is important for hematopoiesis under steady-state conditions, I performed phenotypic studies using flushed BM from either WT or Lep ${ }^{-/}$or Lepr/-mice. FACS analyses of BM collected from Lep ${ }^{-/}$or Lepr/- in comparison to WT mice revealed no significant differences in total BM cellularity (Figure 3.2A), absolute numbers of LT-HSC (Figure 3.2B), ST-HSC (Figure 3.2C), MPP (Figure 3.2D), CMP (Figure 3.2E), MEP (Figure 3.2G) and CLP (Figure 3.2H). Notably, there was a significant increase in the number of GMP in Lepr/- as compared to WT (Figure 3.2F). This suggested that LEP/LEPR axis does not have any roles (direct or indirect) on the maintenance of HSC/HPC populations at least in non-stressed situations. Perhaps, it was true that the isoforms of Lepr expressed by hematopoietic cells were only the truncated nonfunctional ones. 
A.

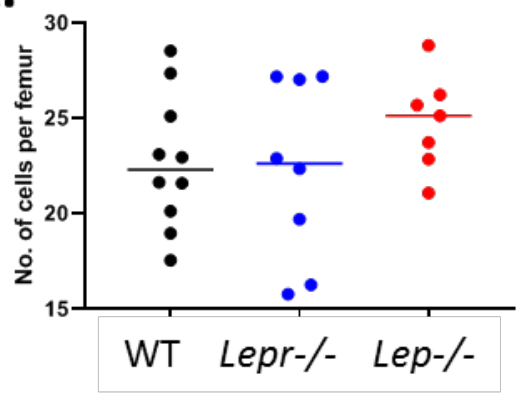

C.

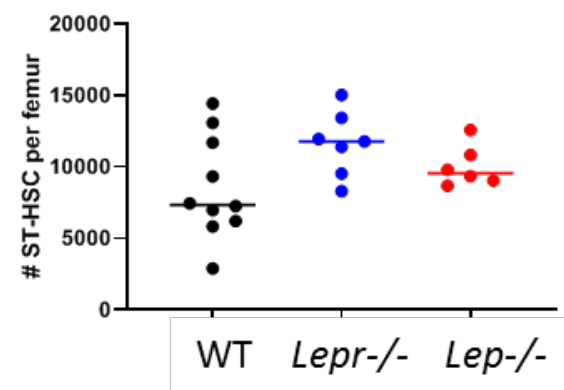

E.

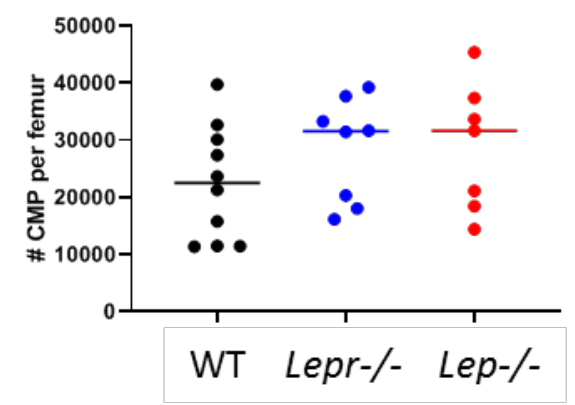

B.

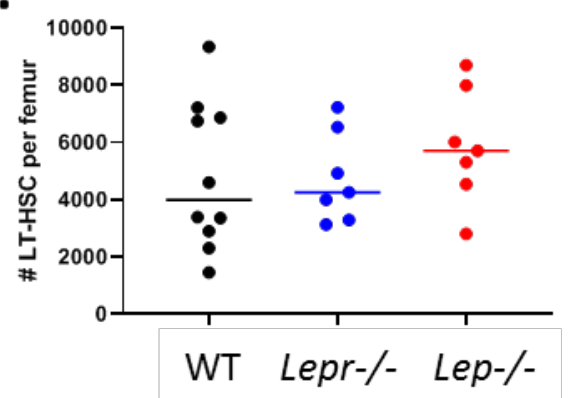

D.

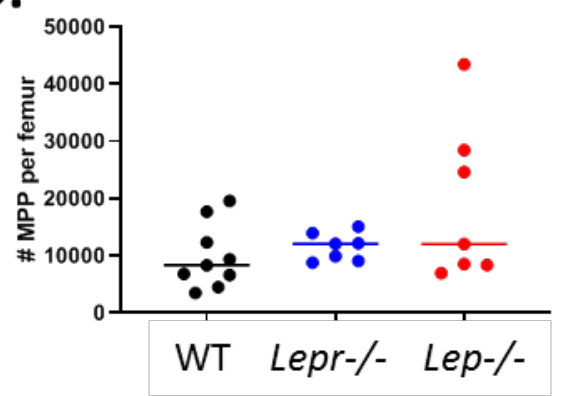

F.

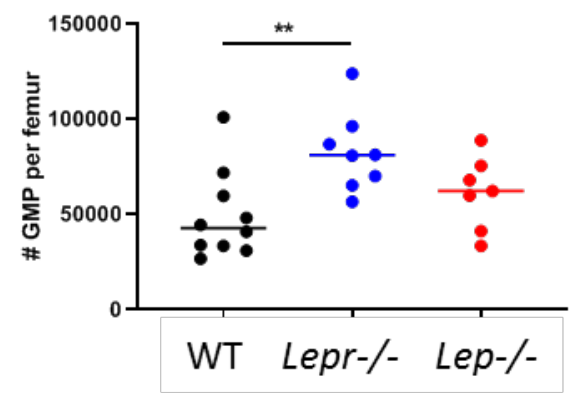



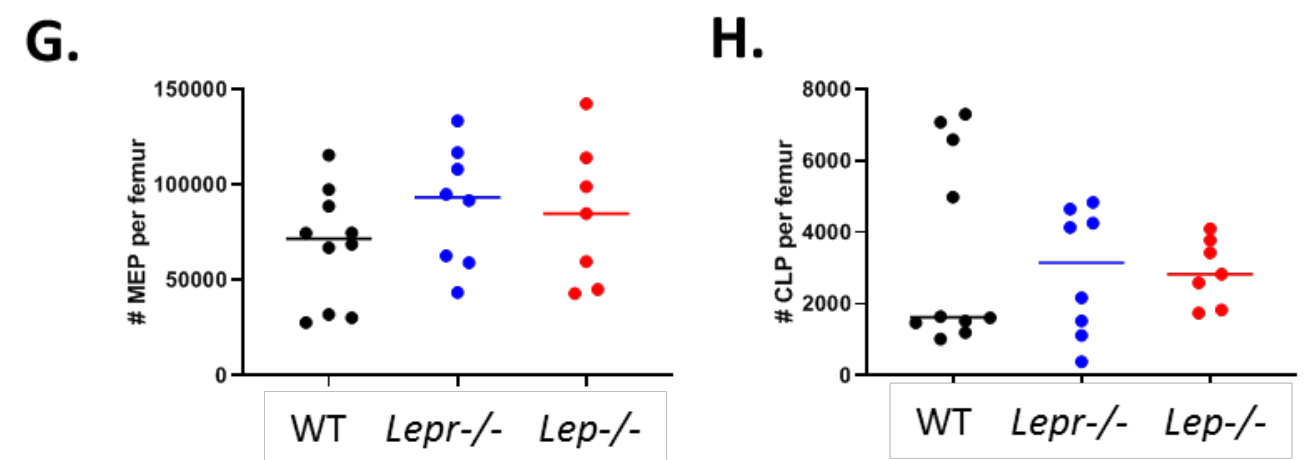

Figure 3.2_Germline Knockout of murine Lep or Lepr did not alter numbers of BM phenotypic HSCs/HPCs.

Freshly isolated BM from femurs of $L e p r^{/-}$or $L e p^{-/}$or wild-type (WT) control were used for FACS phenotyping analyses.
(A) Total cellularity per femur.
(B) Absolute cell numbers of LT-HSCs per femur.
(C) Absolute cell numbers of ST-HSCs per femur.
(D) Absolute cell numbers of MPP per femur.
(E) Absolute cell numbers of CMP per femur.
(F) Absolute cell numbers of GMP per femur.
(G) Absolute cell numbers of MEP per femur.
(H) Absolute cell numbers of CLP per femur.
All data are mean \pm STD. Unpaired Student's t-test was performed. ${ }^{*} p<0.05,{ }^{* *}$ $p<0.01,{ }^{* * *} p<0.001$ compared with WT control.




\subsection{LEPR ${ }^{+}$LSK cells were more highly enriched for phenotypically defined HSCs and MPPs and contained significantly higher numbers of HPCs as compared to LEPR-LSK cells}

As discussed in chapter one, $\mathrm{HCT}$ is a curative treatment for many hematological and non-hematological disorders. Unfortunately, due to their rarity the limited number of HSCs remains as one of the most significant obstacles for advancements in the field. Further complicating this issue is the fact that phenotypic markers used to identify these cells do not always align with their functional properties upon transplantation (Chen et al., 2019). Therefore, the search for a functional marker for HSCs is clinically relevant and crucial.

The data presented in section 3.2 suggested that LEP/LEPR signaling is not required to maintain HSC/HPC pool in murine BM under steady state. However, from a clinical standpoint I am interested in how LEPR-expressing HSCs might behave differently from the LEPR-negative HSCs during the reconstitution process. Here, I sought to determine whether LEPR-expressing HSCs/HPCs were functionally different from their negative counterparts particularly during stressful events such as post-irradiation.

To determine the proportions of SLAM-HSC (LSKCD150+CD48-, hereafter denoted as HSC) and SLAM-MPP (LSKCD150-CD48-, hereafter denoted as MPP) populations that were contained within each compartment ( LEPR ${ }^{+} L S K$ versus LEPR-LSK), I used the gating strategy described in Figure 3.3Ai, and 
found that LEPR ${ }^{+} L S K$ cells were highly enriched for both phenotypically-defined HSCs and MPPs (Figure 3.3Aii). This finding suggested that if LEPR can be used as a reliable marker for functional HSCs and HPCs in vitro as well as in vivo, then LEPR ${ }^{+}$LSK should give rise to more colonies in colony forming unit (CFU) assays and more robust donor engraftment in transplantation assays. 
Ai.

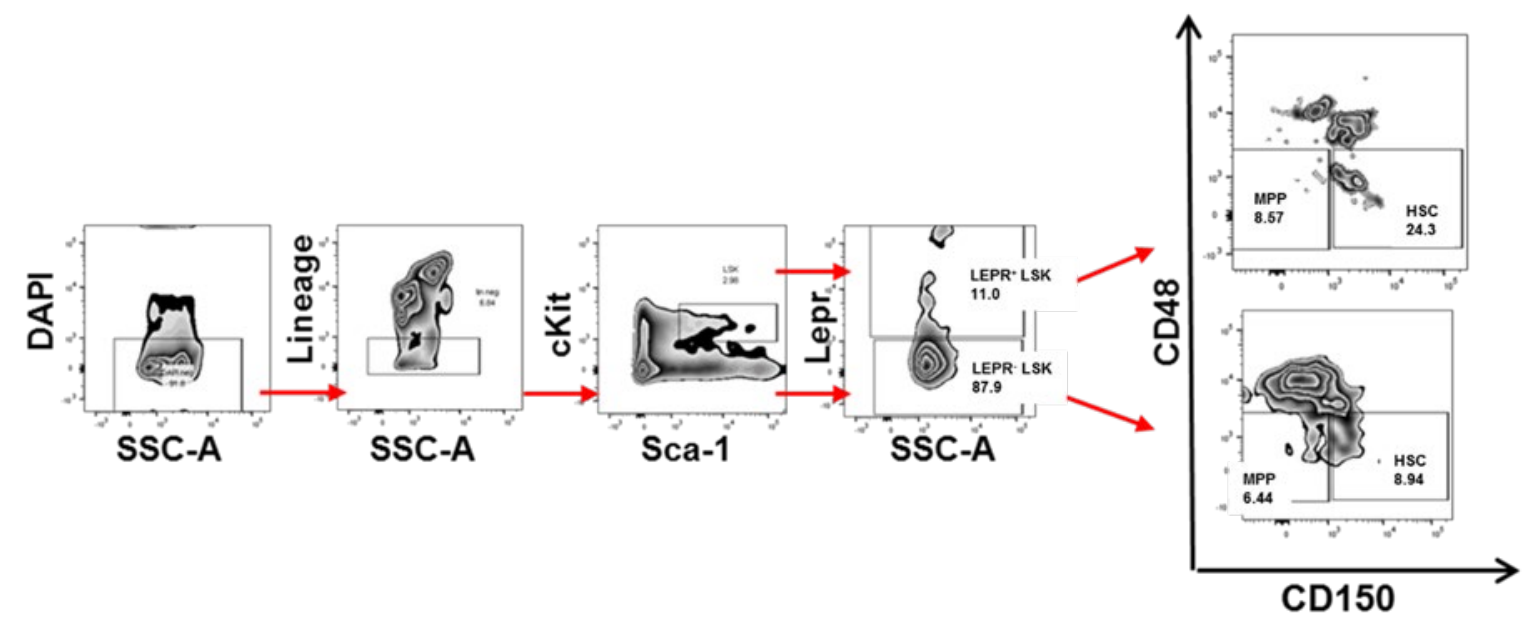

Aii - LEPR $^{+}$LSK •

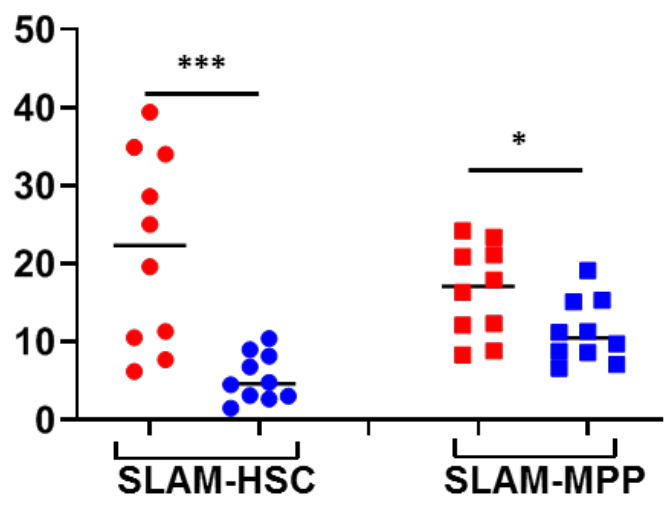


Figure 3.3 LEPR ${ }^{+}$LSK cells were more highly enriched for phenotypically defined HSCs as compared to LEPR-LSK cells.

FACS analyses of freshly isolated BM cells from young adult C57BL/6J mice $(n=8-10)$.

(Ai). Representative gating strategy to determine fractions of SLAM-HSC

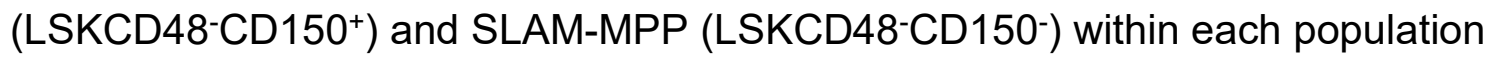
(LEPR+LSK vs. LEPR-LSK).

(Aii). Fractions of SLAM-HSC and SLAM-MPP cells within each population (LEPR+LSK vs. LEPR-LSK).

All data are mean $\pm S D .{ }^{*} p<0.05,{ }^{* * *} p<0.001$ using Mann-Whitney test to compare fractions of SLAM-HSC, SLAM-MPP in (Aii) out of LEPR+ LSK vs. LEPR- LSK cells. $\mathrm{N}=2$ (independently repeated experiments). 
3.4 Compared to LEPR-LSK cells, LEPR ${ }^{+}$LSK cells contained significantly higher numbers of both colony-forming progenitor cells and functional long-term self-renewing HSCs

Compared to LEPR-LSK cells, LEPR ${ }^{+}$LSK cells were significantly enriched for phenotypically identified HSCs and MPPs. Hence, I hypothesized that LEPR ${ }^{+}$LSK cells might outperform LEPR- cells in engraftment studies and/or yield more colonies in CFU assay.

Potential differences in differentiation capacity of LEPR ${ }^{+}$LSK cells with LEPR-LSK cells were assessed using CFU assays. I sorted each population from freshly isolated young adult C57BL/6J BM and plated them in equal numbers $(200-300$ cells/dish). As predicted, LEPR ${ }^{+}$LSK cells produced significantly higher absolute numbers of functional CFU-granulocyte, macrophage (CFU-GM), burst-forming unit-erythroid (BFU-E), and CFU-granulocyte, erythrocyte, macrophage, megakaryocyte (CFU-GEMM) progenitors respectively (Figure 3.4A-C). 

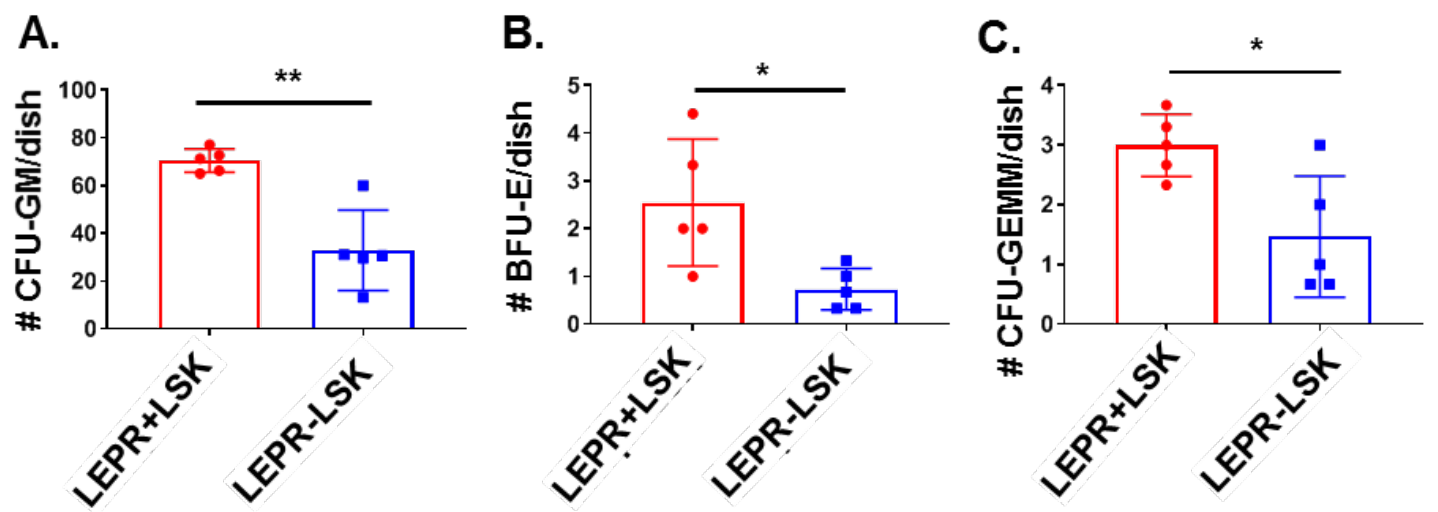


\section{Figure 3.4 Compared to LEPR-LSK cells, LEPR ${ }^{+}$LSK cells contained} significantly higher numbers of colony-forming progenitor cells.

Equal numbers of freshly sorted LEPR+LSK vs. LEPR-LSK cells from C57BL/6J femur BM were plated in triplicate for each mouse in CFU assay.
(A). Absolute numbers of CFU-GM per dish.
(B). Absolute numbers of BFU-E per dish.
(C). Absolute numbers of CFU-GEMM per dish.

Data are mean \pm SD of 5 mice per group plated in triplicate; $N=2$ (independently repeated experiments). Student's $t$ test was used. * $p<0.05,{ }^{* *}$ $p<0.01,{ }^{* * *} p<0.001,{ }^{* * *} p<0.0001$. 
More importantly, to evaluate the engraftment functions of HSCs contained within each population - LEPR ${ }^{+}$LSK vs. LEPR-LSK cells, I performed long-term competitive transplantation assay in which equal numbers of freshly sorted LEPR ${ }^{+}$LSK and LEPR-LSK cells (1000 cells/recipient) from young adult C57BL/6J donor BM (CD45.1-CD45.2+ ${ }^{+}$) were mixed with young adult BoyJ competitor BM cells (CD45.1 $\left.{ }^{+} \mathrm{CD} 45.2^{-}\right)$and intravenously (i.v.) injected into lethally irradiated $\mathrm{F} 1\left(\mathrm{CD} 45.1^{+} \mathrm{CD} 45.2^{+}\right)$mice. Like the CFU results, LEPR ${ }^{+} \mathrm{LSK}$ cells engrafted more robustly as evaluated by donor CD $45.2^{+}$chimerism in the peripheral blood (PB) at month 1, 2, 4, 6, 7 as well as in the BM at month 7 (Figure 3.5A). An independently repeated transplant showed similar results (Figure 3.5B).

To further confirm that mice received LEPR ${ }^{+}$LSK BM cells had better donor engraftment as a result of having more functional HSCs and HPCs using phenotyping analyses, I collected BM from the F1 mouse femurs and analyzed for different phenotypic stem/progenitor populations. Consistently, LEPR ${ }^{+} L S K$ cells had higher absolute numbers of donor CD45.2+ LSK, MEP, CMP and GMP (Figure 3.5C-F respectively). 
A. $\square$ LSK $\square$ LEPR+LSK $\square$ LEPR-LSK

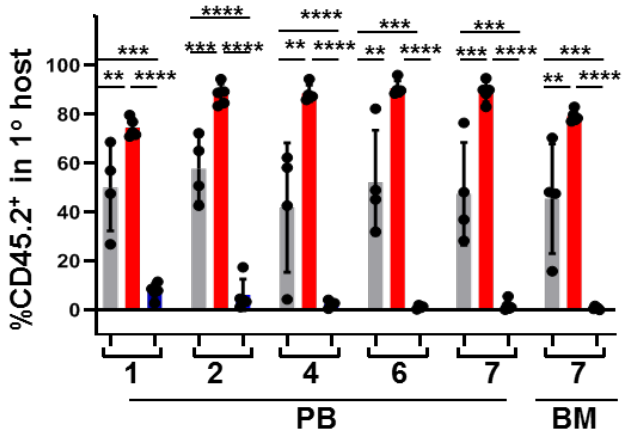

C.

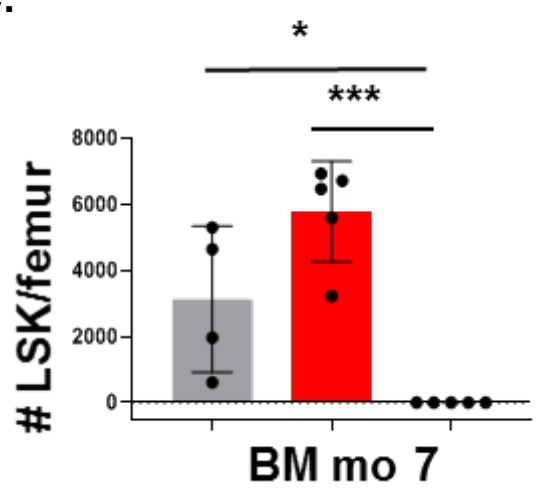

E.

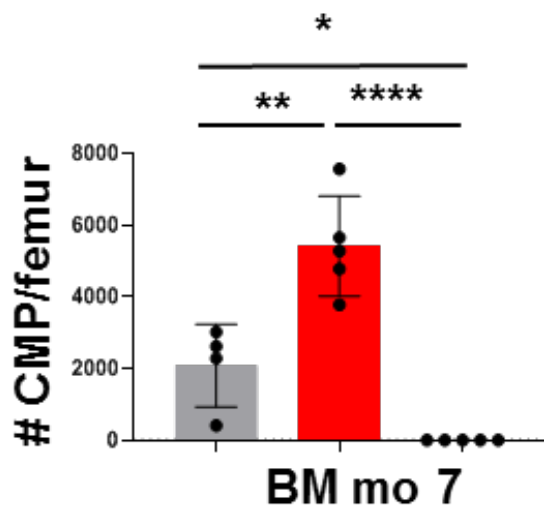

B.

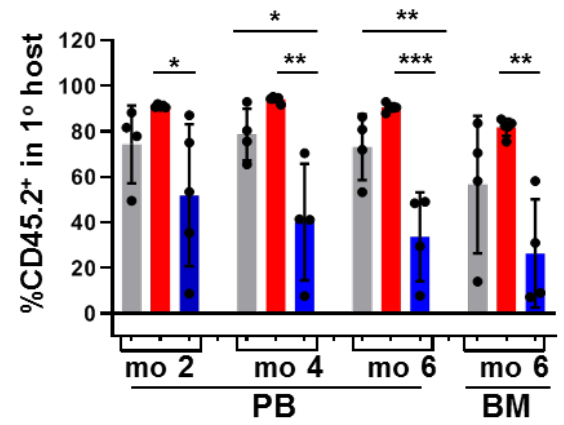

D.

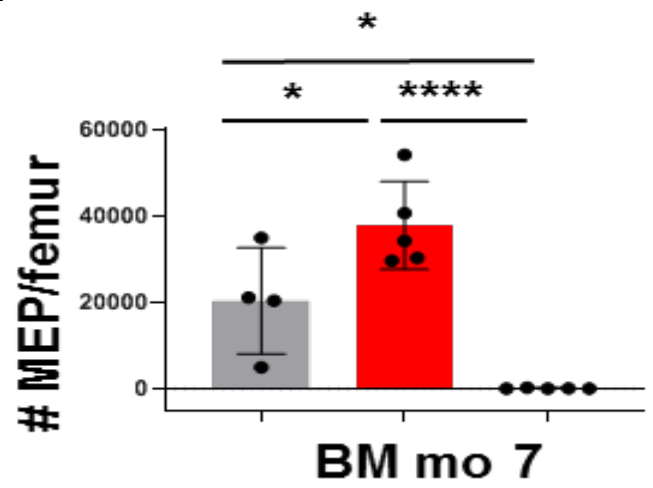

F.

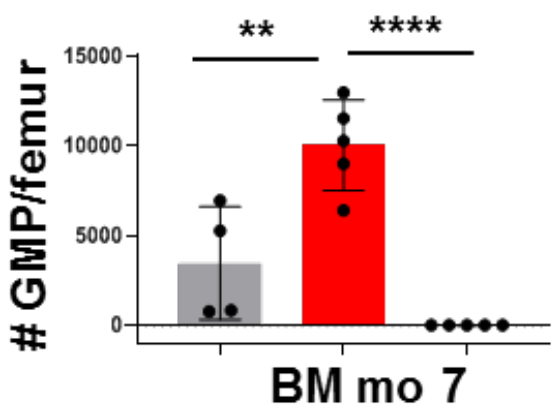


Figure 3.5 Compared to LEPR-LSK cells, LEPR ${ }^{+}$LSK cells were highly enriched for functional long-term HSCs.

Equal numbers of freshly sorted LEPR ${ }^{+}$LSK vs. LEPR-LSK cells from C57BL/6J femur BM were used in competitive transplant assays ( $n=5$ recipients/group). For the primary transplants, BM was pooled from 5 donor mice before sorting.

(A). Percentages of CD45.2 $2^{+}$donor chimerism in PB month 1,2,4,6,7 and BM month 7 respectively.

(B). Percentages of CD45.2 $2^{+}$donor chimerism in PB month 2,4,6 and BM month 6 respectively.

(C-F). Absolute numbers per femur of donor LSK, MEP, CMP and GMP in the BM of primary F1 recipient mice at month 7 respectively.

Data are mean $\pm S D .{ }^{*} p<0.05,{ }^{* *} p<0.01,{ }^{* * *} p<0.001,{ }^{* * *} p<0.0001$ using Ordinary One-way ANOVA followed with post-hoc Tukey's multiple comparison test 
Competitive repopulating units, CRUs, are a functional measure of mouse HSC numbers. To determine actual numbers of functional HSCs, a limiting dilution engraftment assay (LDA) was done. Frequency of CRUs was found to be significantly much higher in LEPR ${ }^{+}$LSK than in LEPR-LSK cells (Figure 3.6A, B). In the limiting dilution transplant, LEPR ${ }^{+}$LSK cells consistently engrafted better than LEPR-LSK as demonstrated by higher CD45.2+ donor chimerisms in PB throughout month 1, 2, 4 and BM month 4 (Figure 3.6Ci-iv respectively). In line with this, FACS analysis of recipient mouse BM at the last time point showed that LEPR ${ }^{+}$LSK group contained significantly higher absolute numbers of donor LSK and MEP cells (Figure 3.6Di-ii respectively) with a trend towards higher numbers of CMP and GMP cells (Figure 3.6Diii-iv respectively). 
A.

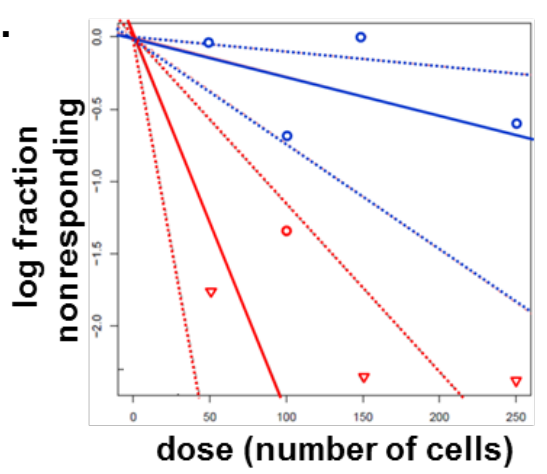

. .LEPR+LSK — .....LEPR-LSK
B.

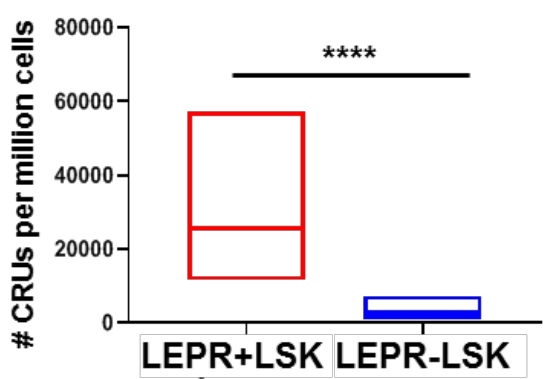

Poisson statistical analysis $p=7.75 e^{-05}$
Ci.

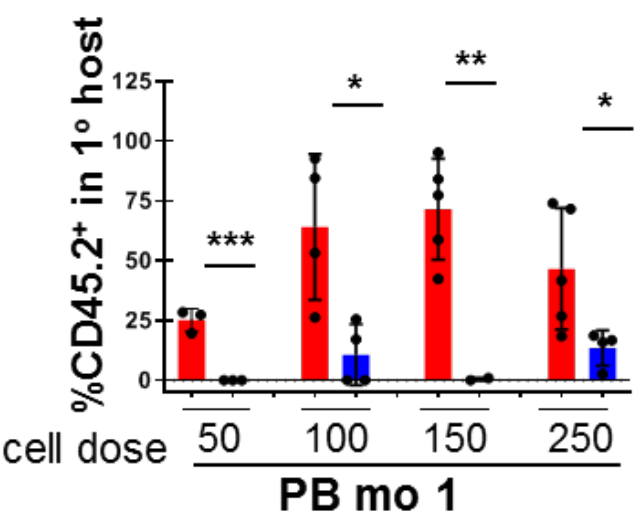

Cii.

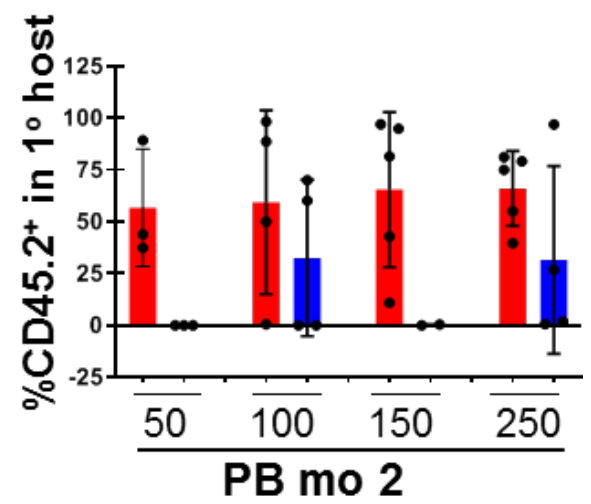

LEPR+LSK $\square$ LEPR-LSK

Ciii.

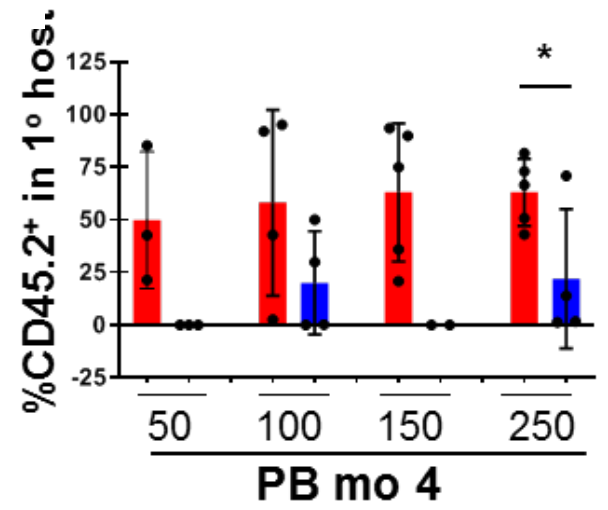

Civ.

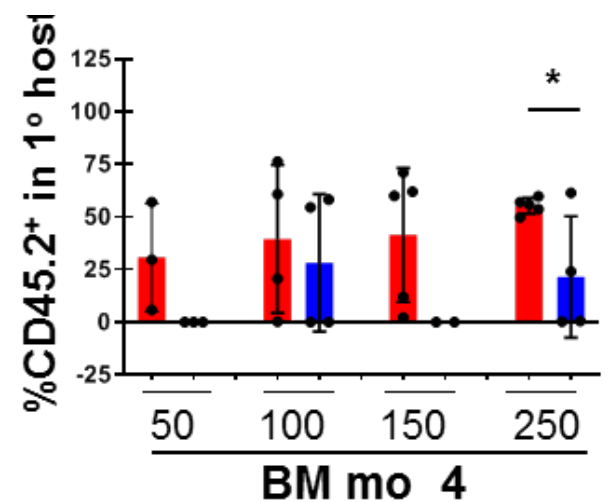


Di.

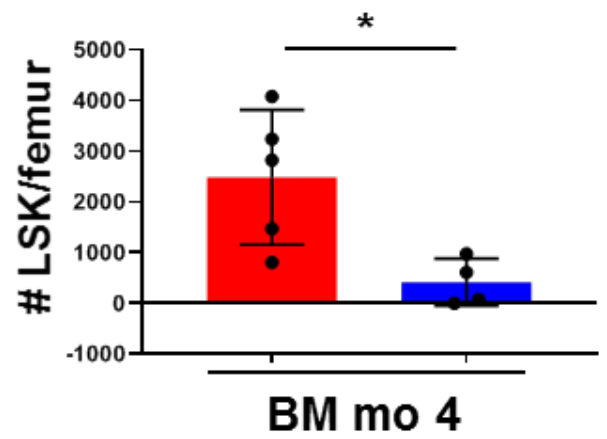

LEPR+LSK םLEPR-LSK

Diii.

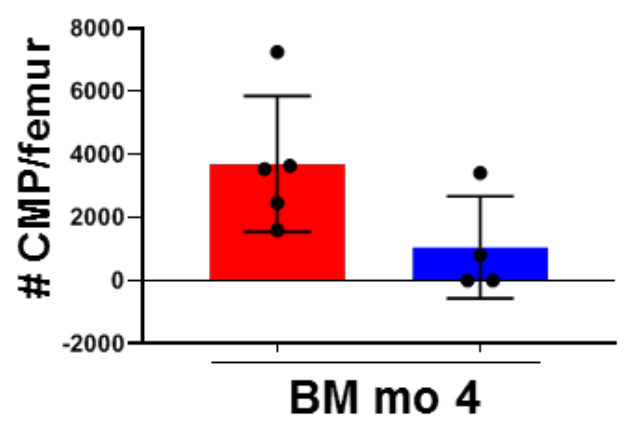

Dii.

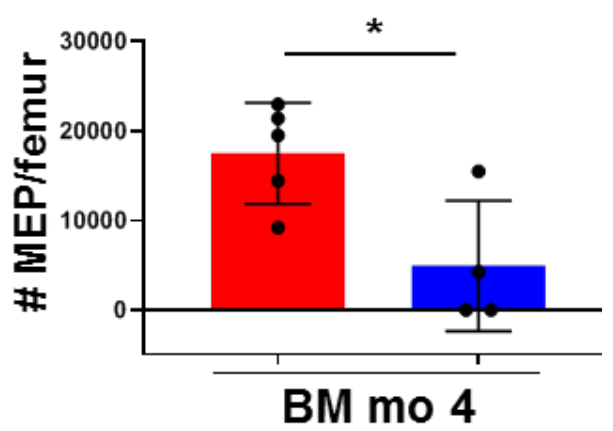

Div.

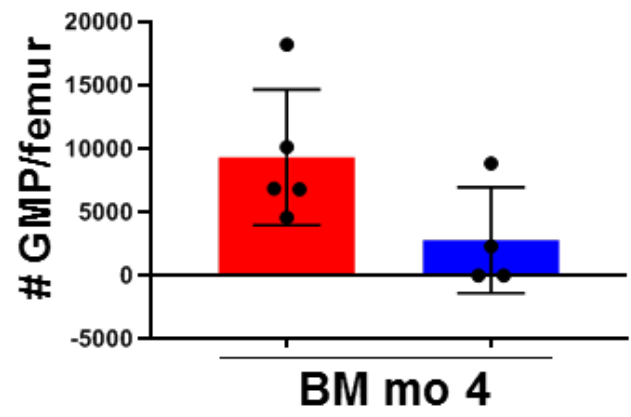


Figure 3.6 LEPR+LSK cells contained significantly higher numbers of competitive repopulating units (CRUs) as compared to LEPR-LSK cells in a limiting dilution assay transplant.

Freshly isolated different doses of donor cells from C57BL6/J (CD45.2 $\left.{ }^{+}\right)$ combined with 100,000 competitor cells from BoyJ $\left(C D 45.1^{+}\right)$were i.v. injected into $F_{1}$ recipients. Engraftment data from month $4 \mathrm{BM}$ was used to calculated CRUs using a cut off value of $1 \%$.

(A). Poisson statistical analysis from the limiting dilution analysis. Solid lines represent the best-fit linear model for each data set. Dotted lines represent 95\% confidence intervals. Symbols represent the percentage of negative mice for each dose of cells. Plot was modified from the original for better clarity.

(B). No. of CRUs per one million transplanted cells calculated from (A). Line indicates median.

(Ci-iv). Percentages of CD45.2 ${ }^{+}$donor chimerism in PB month 1,2,4 and BM month 4 respectively.

(Di-iv). Absolute numbers per femur of donor LSK, MEP, CMP and GMP in the BM of primary F1 recipient mice at month 4 respectively.

Data are mean $\pm S D .{ }^{*} p<0.05,{ }^{* *} p<0.01,{ }^{* * *} p<0.001,{ }^{* * *} p<0.0001$ using Student's $t$ test in (Ci-iv) and (Di-iv). Poisson statistical analysis in (A-B) $p=$ $7.75 \mathrm{e}^{-05}$ 
Self-renewal is one of the hallmark characteristics of functional long-term HSCs. To assess the ability of self-renewing of LEPR ${ }^{+} H S C$ s as compared to LEPR HSCs, at the last time-point of each primary transplant, I collected BM from the F1 recipient mice and transplanted them into lethally irradiated secondary hosts. Consistent with primary transplant results, LEPR ${ }^{+}$LSK cells engrafted much more extensively than LEPR-LSK cells in the PB throughout month 1, 2, 4, 5 and BM month 5 of the secondary transplant (Figure 3.7A). The same trends were observed in secondary transplants of an independently repeated experiment (Figure 3.7B). 
A. $\square$ LSK $\square$ LEPR+LSK $\square$ LEPR-LSK

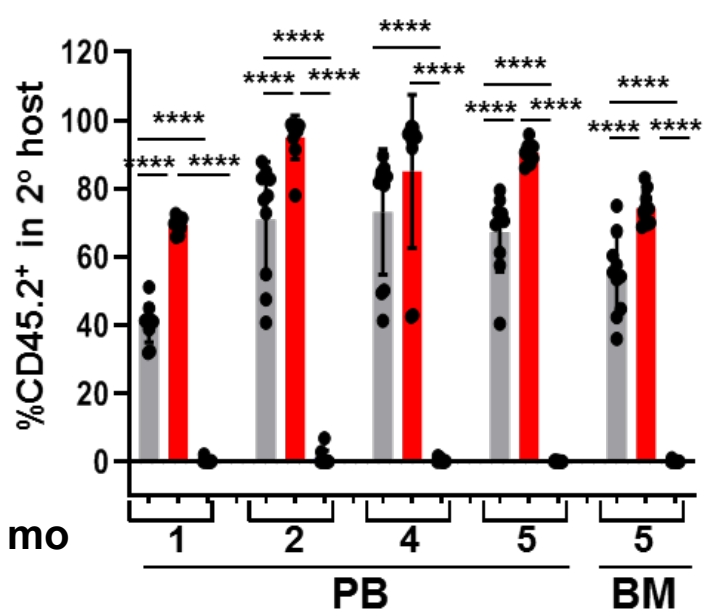

B.

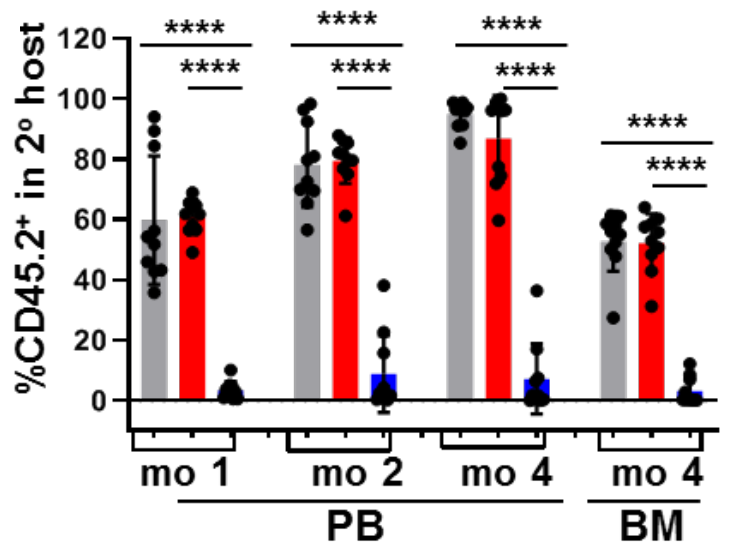


Figure 3.7 Compared to LEPR-LSK cells, LEPR+LSK cells were more highly enriched for self-renewing HSCs as shown in secondary transplants.

Whole BM flushed from primary F1 recipients were pooled within each group and i.v. injected into secondary recipients ( $\mathrm{n}=10$ recipients).

(A). Percentages of CD45.2 $2^{+}$donor chimerism in secondary mice in the PB month 1, 2, 4, 5 and BM month 5.

(B) Percentages of CD45.2 $2^{+}$donor chimerism in secondary mice in the PB month 1, 2, 4 and BM month 4 of an independently repeated experiment.

Data are mean $\pm S D$. ${ }^{*} p<0.05,{ }^{* *} p<0.01,{ }^{* * *} p<0.001,{ }^{* * *} p<0.0001$ using Ordinary One-way ANOVA followed with post-hoc Tukey's multiple comparison test. 


\subsection{LEPR differentiates SLAM HSCs into two functionally distinct}

populations with LEPR ${ }^{+} H S C$ s, a smaller subset but with significantly higher repopulating potential

Members of the SLAM family markers, CD150 and CD48, are arguably the most widely used markers for long-term functional HSCs (Oguro, Ding, \& Morrison, 2013). To elucidate whether LEPR can functionally classify SLAM HSC (LSKCD150 ${ }^{+}$CD48) into two distinct subpopulations, I performed a competitive limiting dilution transplantation assay in which three different doses of freshly sorted LEPR+HSC or LEPR-HSC cells from young adult C57BL6/J donor BM (CD45.1 ${ }^{-C D} 45.2^{+}$) combined with unseparated young adult BoyJ competitor cells $\left(C D 45.1^{+} \mathrm{CD} 45.2^{-}\right)$were i.v. injected into lethally irradiated F1 mice $\left(C D 45.1^{+} \mathrm{CD} 45.2^{+}\right)$. Interestingly, LEPR ${ }^{+} \mathrm{HSC}$ s possessed superior engrafting capability as compared to LEPR-HSCs as shown by statistically significantly higher donor CD45.2+ chimerism in the PB at month 1, 2, 4 and BM month 4 (Figure 3.8Ai-iv respectively). In fact, using Poisson statistical analysis I found that LEPR ${ }^{+} H S C$ s were significantly enriched for CRUs as compared to LEPR HSCs (Figure 3.8B, C). 
Ai.

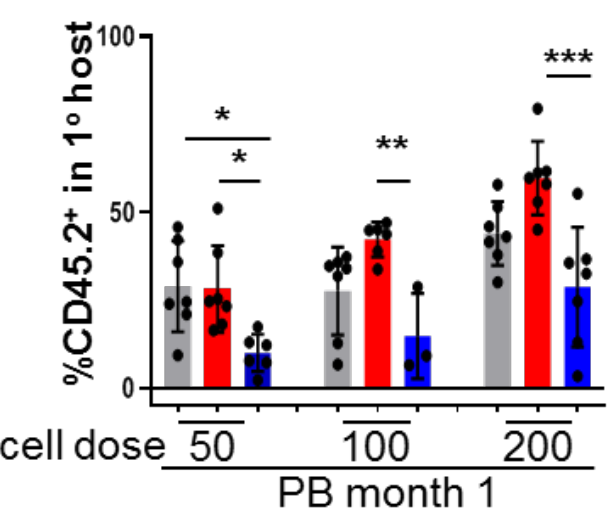

Aii.

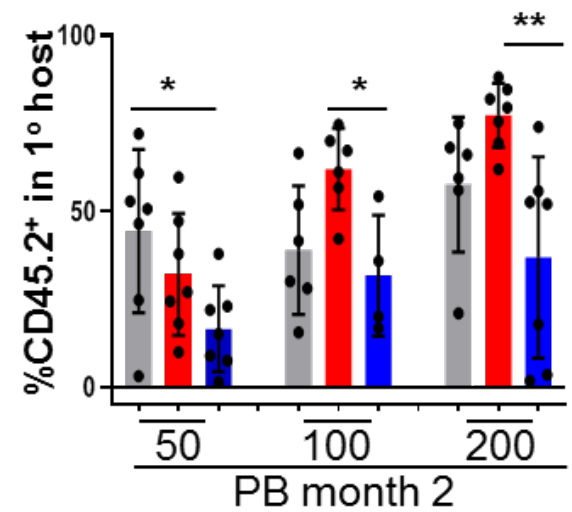

\section{HSC $\square$ LEPR $^{+}{ }^{+}$SC}

Aiii.

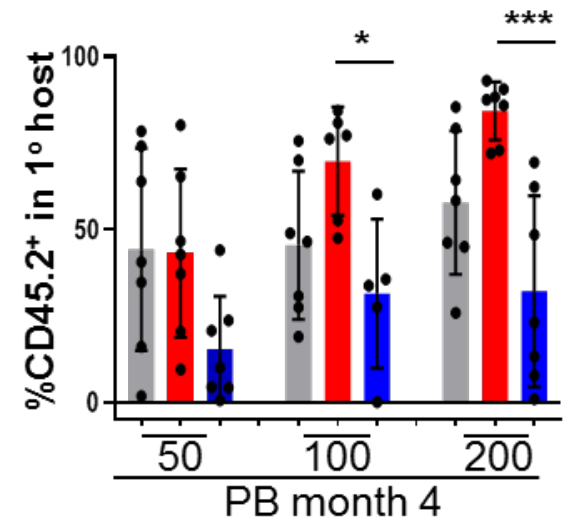

Aiv.

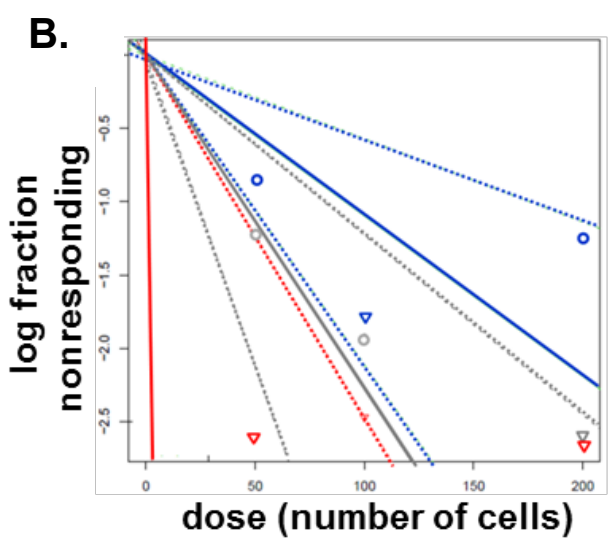

c.

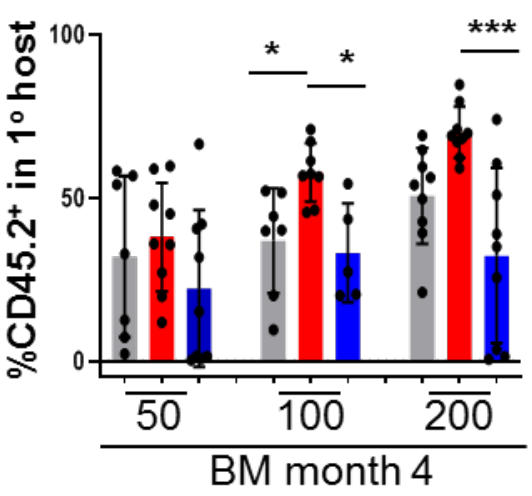

Poisson analysis $p=0.00172$

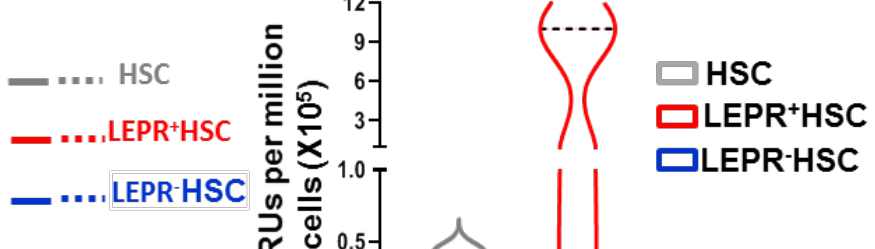


Figure 3.8 LEPR differentiates SLAM HSCs into two functionally distinct populations with LEPR ${ }^{+} H S C$ s, a smaller subset but with significantly higher repopulating potential.

Different doses of LEPR+HSCs and LEPR-HSCs were sorted from freshly isolated and pooled BM cells from C57BL/6J femurs ( $\mathrm{n}=15$ mice) and used in a limiting dilution assay transplant ( $\mathrm{n}=7$ recipients/group).

(Ai-iv). Percentages of CD45.2 $2^{+}$donor chimerism in PB month 1, 2, 4 and BM month 4 respectively.

(B). Poisson statistical analysis from the limiting dilution analysis. Solid lines represent the best-fit linear model for each data set. Dotted lines represent 95\% confidence intervals. Symbols represent the percentage of negative mice for each dose of cells. Plot was modified from the original for better clarity.

(C). No. of CRUs per one million transplanted cells calculated from (B). Line indicates median.

Data are mean $\pm S D$. ${ }^{*} p<0.05,{ }^{* *} p<0.01,{ }^{* * *} p<0.001,{ }^{* * * *} p<0.0001$ using Ordinary One-way ANOVA followed with post-hoc Tukey's multiple comparison test in (Ai-iv). Poisson statistical analysis in (D-E) $p=0.00172$. 
In addition, FACS analysis of BM month 4 confirmed that mice received LEPR ${ }^{+} H S C$ s contained significantly higher numbers of donor LT-HSC, MPP, MEP, CMP and GMP (Figure 3.9A, C-F respectively) and a trend towards higher numbers of ST-HSC (Figure 3.9B). 
A.

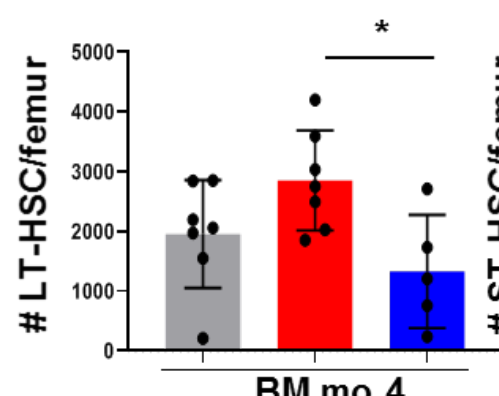

BM mo 4
B.

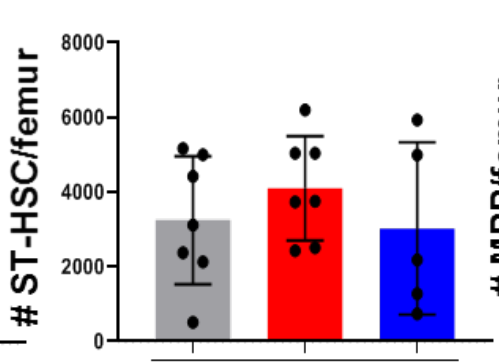

BM mo 4

C.

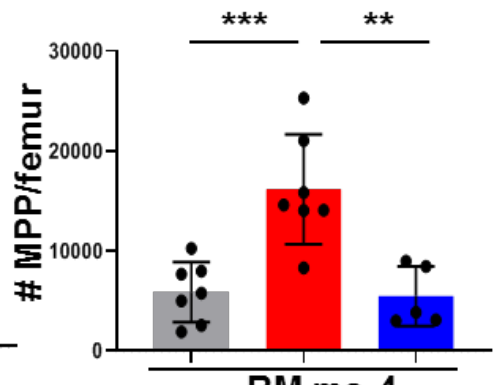

BM mo 4

D.

E.

F.

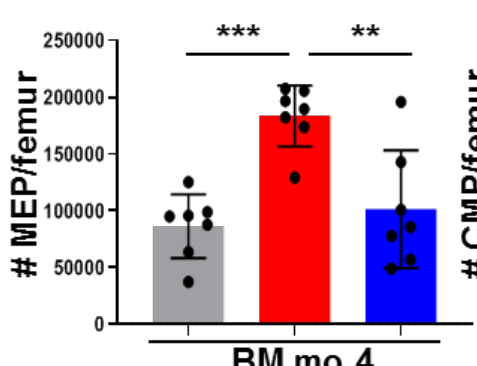

BM mo 4
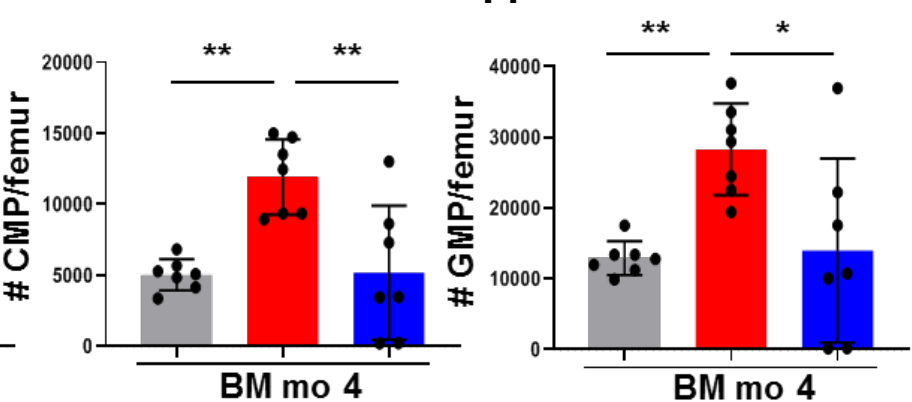

BM mo 4 
Figure 3.9 BM from recipient mice transplanted with LEPR ${ }^{+} H S C s$ were significantly enriched with HSCs and HPCs.

Different doses of LEPR+HSCs and LEPR-HSCs were sorted from freshly isolated and pooled BM cells from C57BL/6J femurs ( $\mathrm{n}=15$ mice) and used in a limiting dilution assay transplant ( $n=7$ recipients). FACS analyses of recipient BM at month 4 .

(A - F) Absolute cell numbers of LT-HSCs, ST-HSCs, MPP, MEP, CMP, and GMP per femur respectively.

Data are mean $\pm S D$. ${ }^{*} p<0.05,{ }^{* *} p<0.01,{ }^{* * *} p<0.001,{ }^{* * *} p<0.0001$ using Ordinary One-way ANOVA followed with post-hoc Tukey's multiple comparison test. 
3.6 LEPR ${ }^{+}$HSCs did not exhibit a lineage bias in competitive transplantation assays, shared similar homing capacity and cycling status with LEPR-HSCs

The pleiotrophic effects of leptin functions of mature immune cells both of the myeloid and lymphoid branches have been well-recognized (Naylor \& Petri, 2016). To determine whether LEPR ${ }^{+} H S C$ s have any biases in lineage fate decision, I analyzed the percentages of donor B220 $\mathrm{B}$ cells, CD3e ${ }^{+} \mathrm{T}$ cells and CD11 $\mathrm{b}^{+}$myeloid cells in both PB and BM at the last time points of primary transplants. Compared to the LEPR-HSCs, LEPR ${ }^{+} H S C$ donor cells had similar percentages of $B 220^{+} B$ cells, CD3e+ $e^{+}$cells and $C D 11 b^{+}$myeloid cells in PB and BM month 4 in all three doses of a limiting dilution assay (Figure 3.10Ai-iii). The same trend was observed in two other transplants (Figure 3.10B, C). Hence, we concluded that LEPR ${ }^{+} H S C$ s had a similar lineage output with LEPR-HSCs and total HSCs. 
Ai.

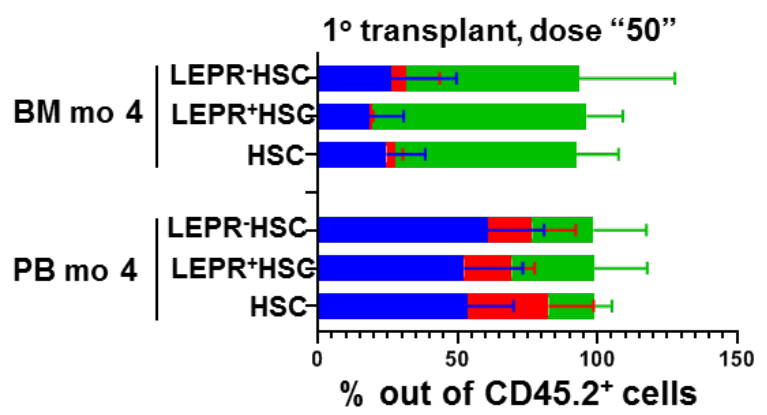

Aii.

10 transplant, dose "100"

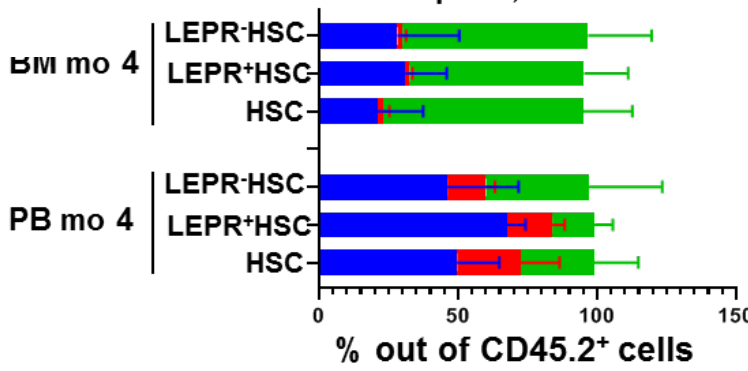

Aiii

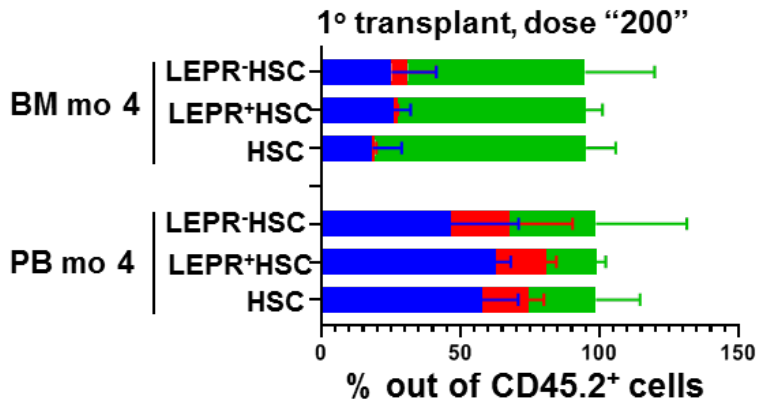

B.

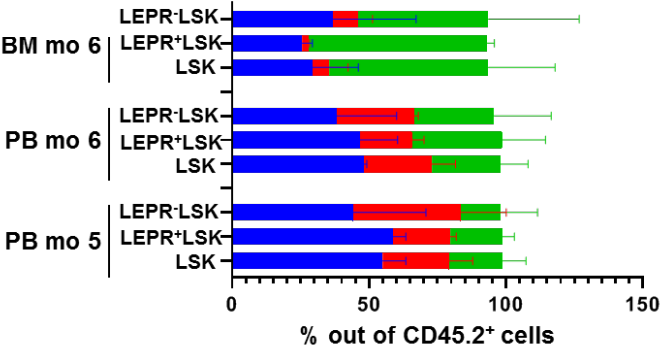

c.

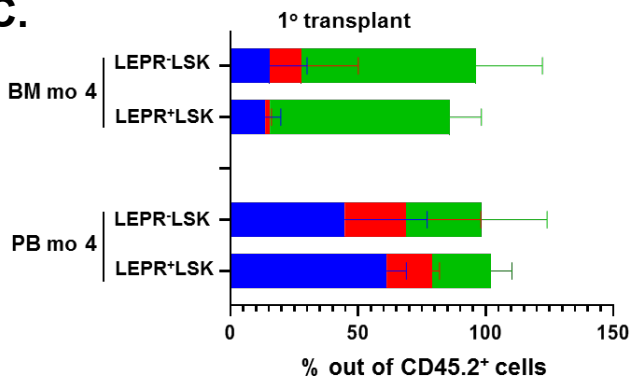

- CD11b $b^{+}$Myeloid $=\mathrm{CD}^{+} \mathrm{e}^{+} \mathrm{C} 220^{+} \mathrm{B}$ 
Figure 3.10 LEPR ${ }^{+} H S C s$ had a similar lineage output with LEPR-HSCs. (Ai-iii) Three different doses of freshly sorted LEPR ${ }^{+} H S C$ vs. LEPR-HSC cells were used in a limiting dilution assay transplant. Percentages of donor $\mathrm{B}_{22} \mathrm{O}^{+} \mathrm{B}$, $\mathrm{CD} \mathrm{e}^{+} \mathrm{T}$ and $\mathrm{CD} 11 \mathrm{~b}^{+}$myeloid cells in primary $\mathrm{F} 1$ hosts in PB month 4 and BM month 4 in three doses.

(B) Equal numbers of freshly sorted LEPR ${ }^{+} L S K$ vs. LEPR-LSK cells were used in a regular straight-up transplant assay. Percentages of donor $\mathrm{B} 220^{+} \mathrm{B}, \mathrm{CD} 3 \mathrm{e}^{+} \mathrm{T}$ and CD11 $\mathrm{b}^{+}$myeloid cells in primary F1 hosts in PB month 5, 6 and BM month 6 . $N=2$

(C). Different doses of freshly sorted LEPR ${ }^{+} L S K$ vs. LEPR'LSK cells were used in a limiting dilution assay transplant. Percentages of donor $B 220^{+} \mathrm{B}, \mathrm{CD} 3 \mathrm{e}^{+} \mathrm{T}$ and CD11 $\mathrm{b}^{+}$myeloid cells in primary F1 hosts in PB month 4 and BM month 4.

Data are mean $\pm S D$. Ordinary One-way ANOVA followed with post-hoc Tukey's multiple comparison test and in (Ai-iii, B) and Student's t test in (C). 
Upon being injected into the hosts, HSCs need to migrate to and lodge into the BM niche for engraftment to happen. During this process, expression of CXCR4 by HSCs allow the cells to sense and follow the CXCL12 (or SDF1) chemotaxic gradient by the BM niche cells. This early phase of engraftment is termed 'homing,' and increase in homing could be one factor that contributed to enhanced repopulating outcome. As a result, since enhanced engraftment could be due to increased numbers of cells homed to the BM, I performed a homing assay by i.v. injecting equal numbers of freshly sorted LEPR ${ }^{+}$LSK and LEPR-LSK cells from young adult C57BL6/J donor BM (CD45.1-CD45.2+) into lethally irradiated BoyJ (CD45.1 ${ }^{+}$CD45.2 $)$hosts. Seventeen hours later, BM was flushed from the recipient femurs and stained for FACS analyses. LEPR ${ }^{+} L S K$ and LEPR LSK cells were found to home at similar capacities, as shown by similar numbers of homed LK, LSK and LSKCD150+ cells (Figure 3.11Ai-iii respectively). In line with this, there was no significant difference in CXCR4 surface staining between LEPR+HSC, LEPR ${ }^{+}$MPP as compared to LEPR-HSC and LEPR-MPP cells (Figure 3.11Bi-ii). 
Ai.

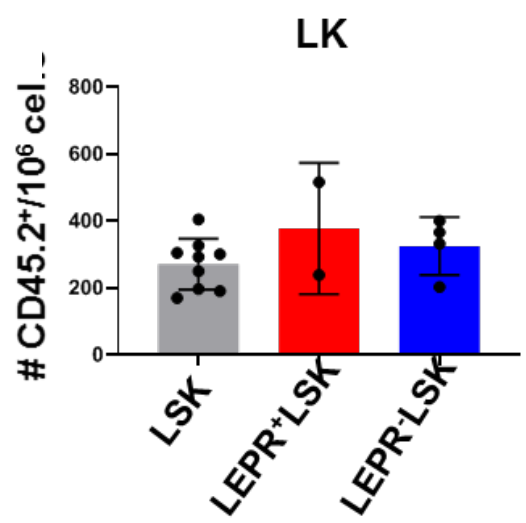

Aii.

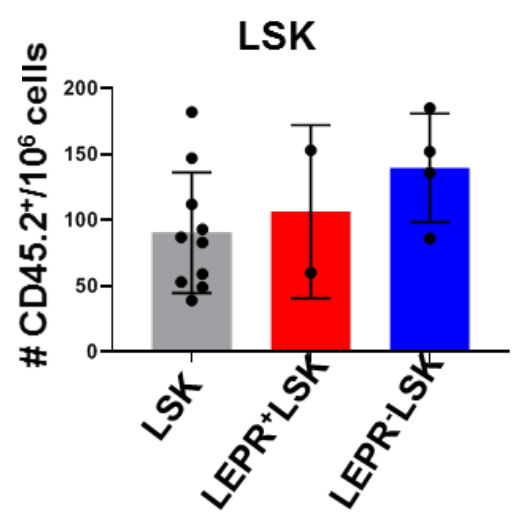

Aiii

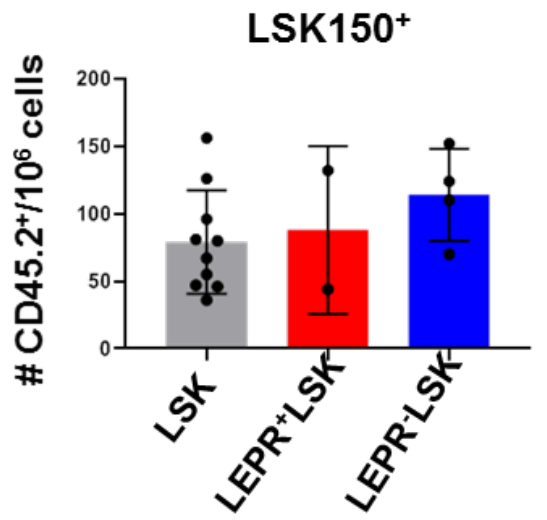

Bi

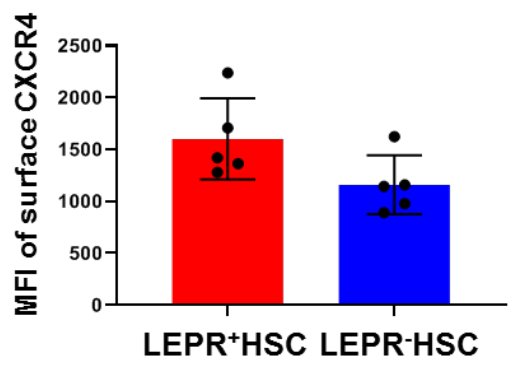

Bii.

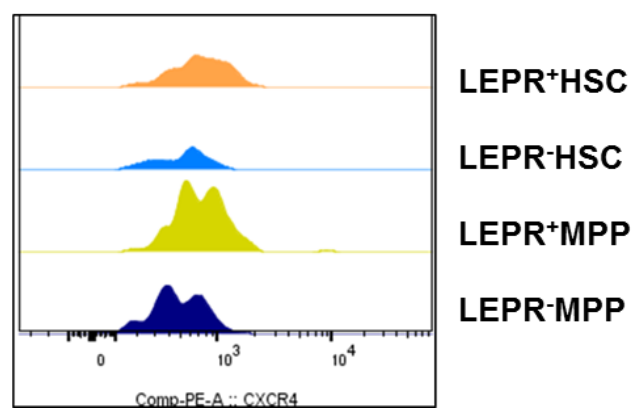


Figure 3.11 LEPR ${ }^{+}$HSCs homed to the BM niche at a similar capacity and shared similar levels of CXCR4 expression with LEPR-HSCs.

(Ai-iii) Equal numbers (7000 cells) of freshly sorted BM LEPR+LSK and LEPRLSK were i.v. injected into lethally irradiated BoyJ recipient mice. BM was flushed from their femurs and immunophenotypically analyzed 17 hours post-injection. Absolute numbers of donor CD45.2+ LK, LSK and LSKCD150+ cells were determined.

(Bi) Surface staining (or mean fluorescence intensity) of CXCR4 on LEPR ${ }^{+} H S C$ and LEPR-HSC.

(Bii) Histograms of level of expression of surface CXCR4 on LEPR ${ }^{+} H S C$, LEPR+MPP as compared to LEPR-HSC, and LEPR-MPP.

Data are mean $\pm S D$. Ordinary One-way ANOVA followed with post-hoc Tukey's multiple comparison test and in (Ai-iii) and Student's t test in (Bi). 
Being protected in a hypoxic microenvironment, it is widely accepted that longterm HSCs are mostly quiescent and rarely divide to avoid replicating exhaustion and uncontrolled differentiation due to exposure to the harmful ROS. Hence, I determined the cycling status of LEPR ${ }^{+} \mathrm{HSCs}_{\text {MPP }}$ cells as compared to LEPR ${ }^{-}$ HSCs/MPP cells by using phenotypic FACS studies. In addition, I also studied the cycling characteristics of more mature progenitor cells through using radioactive high specific activity tritiated thymidine in CFU assay. Both LEPR ${ }^{+}$ functional CFU-GM progenitor cells (assessed by high specific activity tritiated thymidine kill CFU assay) (Figure 3.12A) and LEPR ${ }^{+} H S C$ s and LEPR ${ }^{+}$MPPs (assessed by phenotype) had similar percentages of cells in cycle compared to LEPR- cells (Figure 3.12Bi-ii respectively). 
A.

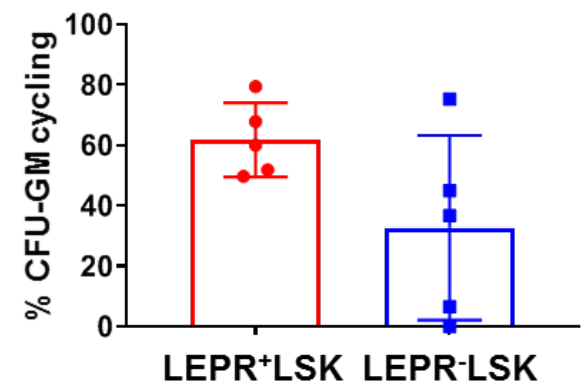

Bi.

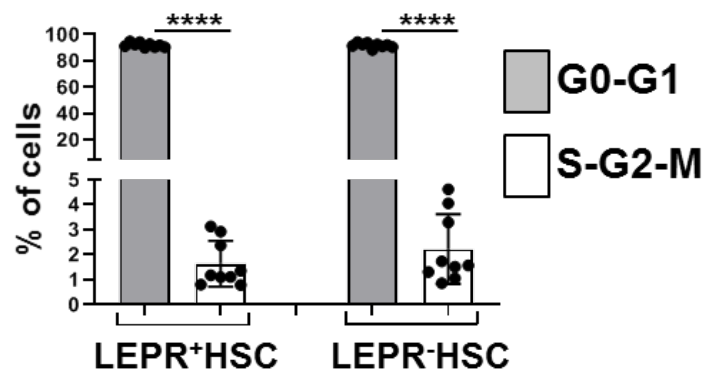

Bii.

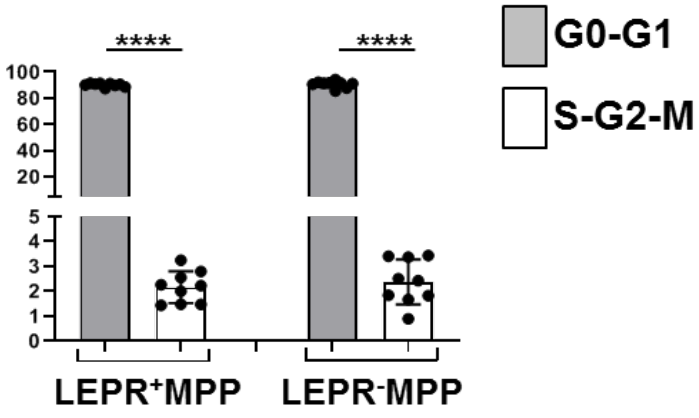


Figure 3.12 LEPR ${ }^{+}$HSCs shared similar cycling status with LEPR-HSCs. (A) Equal numbers of freshly sorted BM LEPR ${ }^{+}$LSK and LEPR-LSK from each mouse ( $n=5$ mice) were plated in triplicate in a high specific activity tritiated thymidine kill CFU assay. Percentages of CFU-GM progenitor cells in cycle for each population.

(Bi-ii) Percentages of cells in different phases of the cell cycle for LEPR ${ }^{+} H S C s$ (or LEPR ${ }^{+}$MPP) vs. LEPR-HSCs (or LEPR-MPP). $\mathrm{N}=2$ (independently repeated experiments).

Data are mean $\pm S D .{ }^{* * * *} p<0.0001$ Student's t test. 


\subsection{LEPR ${ }^{+}$HSCs constitute a subset of functional long-term repopulating}

HSCs that is characterized by a pro-inflammatory transcriptomic signature To delineate the potential transcriptomic networks that modulate the functional differences between LEPR ${ }^{+}$HSC/MPP and LEPR-HSC/MPP cells, I sorted fresh BM cells for each population and used them for bulk RNA-sequencing.

Using a non-biased data-driven approach, heatmap analysis showed a total of 564 genes expressed at higher levels and 511 genes expressed at lower levels in LEPR+HSC compared to LEPR-HSC (Figure 3.13 A). We also found that LEPR ${ }^{+}$MPP contained 409 genes more highly expressed and 130 genes less expressed compared to LEPR-MPP (Figure 3.13C, D). Interestingly, LEPR ${ }^{+} H S C$ cells expressed at higher levels genes involved in megakaryocytopoiesis such as Mpig6b, Plekhb1, and Tubb1. In addition, log2(fold change) of expression level analysis showed that LEPR ${ }^{+} H S C$ s more highly expressed genes encoding cytoskeleton and structural/matrix proteins (Cdc42ep1, Lnx1, Fbln5, Nid1), as well as genes related to cell proliferation, differentiation, adhesion and motility (Lrg1, Hoxb7, Chrdl1, Sema4f) (Figure 3.13B). Notably, both LEPR+HSC and LEPR ${ }^{+} M P P$ cells expressed significantly higher levels of LEPR as an internal quality controls (yellow dot Figure 3.13A, C respectively). 
A.

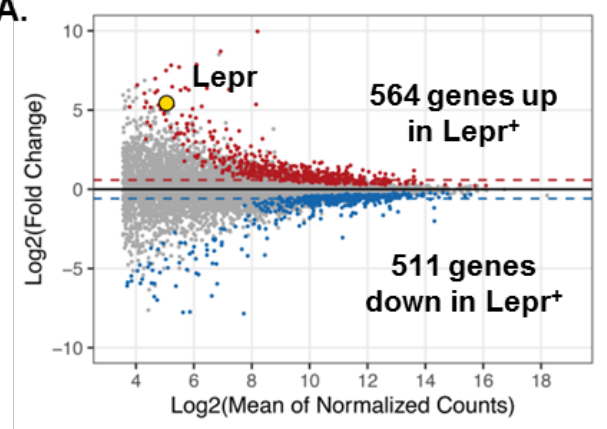

B.
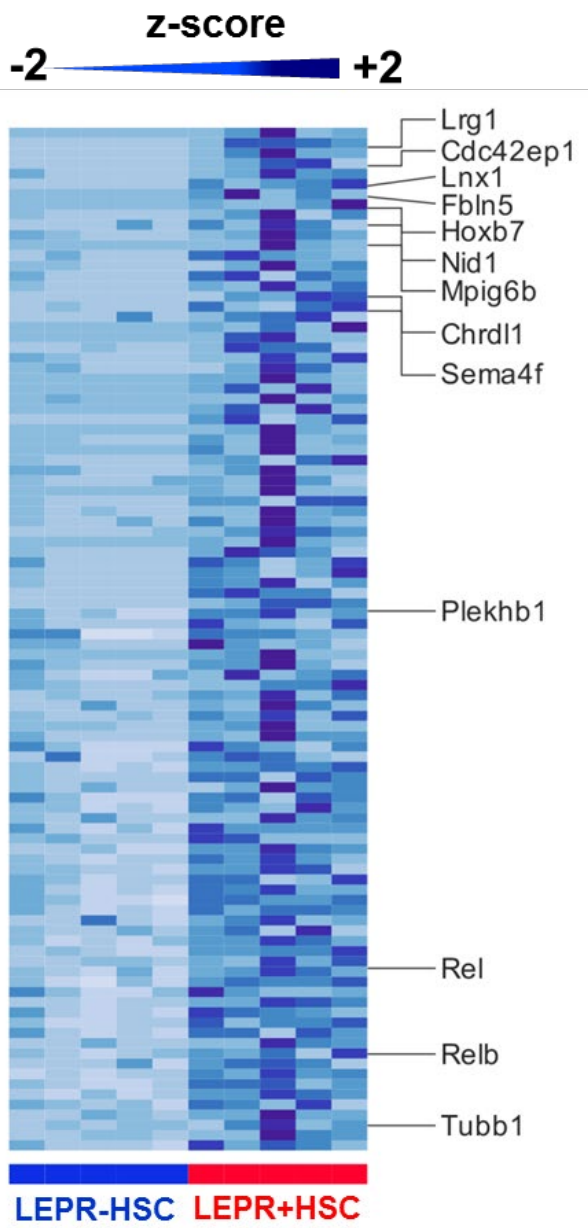

c.

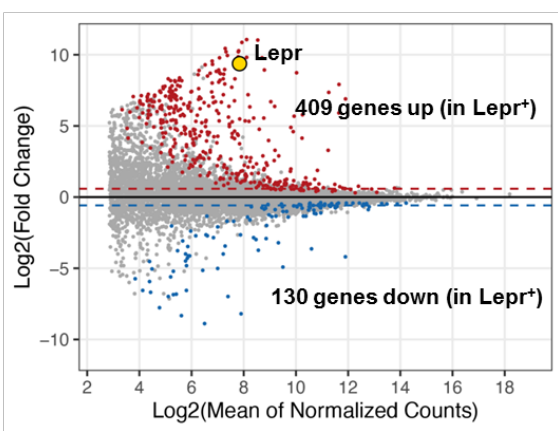

D.
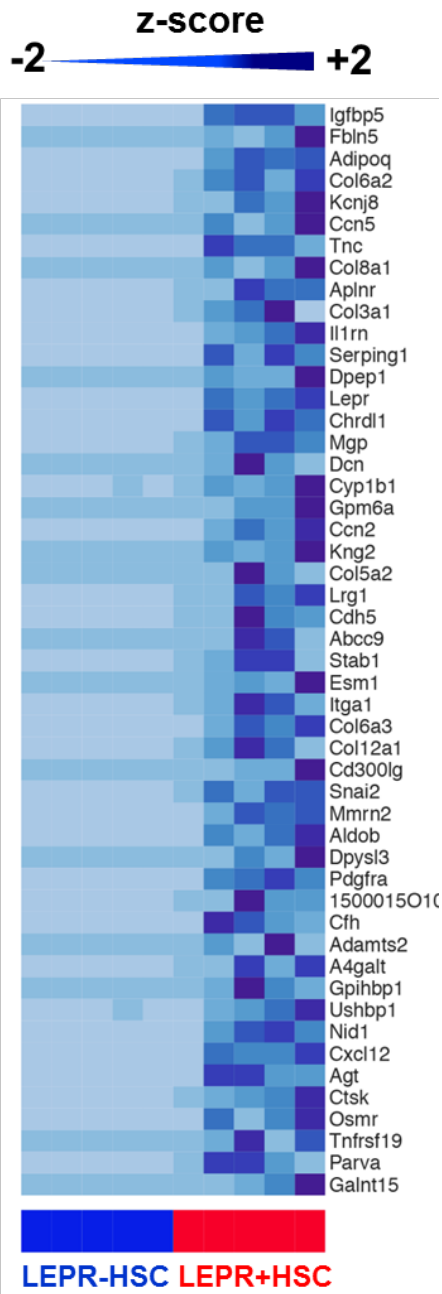

This was done with help from Dr. James Ropa. 
Figure 3.13 LEPR ${ }^{+} \mathrm{HSC} / \mathrm{MPPs}$ are transcriptomically distinctive from LEPRHSCs/MPPs.

LEPR ${ }^{+} H S C$ S/MPPs and LEPR-HSCs/MPPs were sorted from freshly isolated BM from C57BL6/J directly into 2- $\beta$ mercaptoethanol-supplemented lysis buffer and subjected to bulk RNA-sequencing ( $n=5$ biological replicates/population). A positive enrichment score indicates the gene program is enriched in LEPR ${ }^{+}$cells and vice versa.

(A, C). Bland-Altman plots (MA plot) showed fold changes of genes in LEPR+HSCs/LEPR-HSCs in (A) (or LEPR+MPPs/LEPR-MPPs in (C)) versus the average expression of those genes in all samples. Red represented genes that are significantly expressed at higher level in LEPR ${ }^{+} H S C s / M P P s ;$ blue represented genes that are significantly expressed at lower level in LEPR' HSCs/MPPs.

(B). Heatmap representation of the top 100 more highly expressed genes in LEPR ${ }^{+} H S C$ s than LEPR-HSCs; genes discussed in the text are listed to the right of the heatmap. The data is calculated by row. Light-blue color indicates lower row z-score; dark-blue color indicates higher row z-score.

(D). Heatmap representation of the top 50 more highly expressed genes in LEPR ${ }^{+}$MPPs than LEPR-MPPs, which are listed to the right of the heatmap. The data is calculated by row. Light-blue color indicates lower row z-score; dark-blue color indicates higher row z-score. 
To further determine which isoform(s) were expressed, we performed additional quantitative analyses by dividing exons into counting bins using unique annotated transcripts and reads being assigned to exon bins. Results showed LEPR ${ }^{+} H S C / M P P$ expressed predominantly the short isoforms Lepr-a (or Ob-Ra) and Lepr-c (or Ob-Rc) but not the long isoform Lepr-b (or Ob-Rb), which was essentially undetectable (Figure 3.14A, B). This suggested that the LEPR expressed by these cells might not be capable of fully transducing intracellular signaling at least under steady state conditions as when the cells were collected and extracted for RNA. 


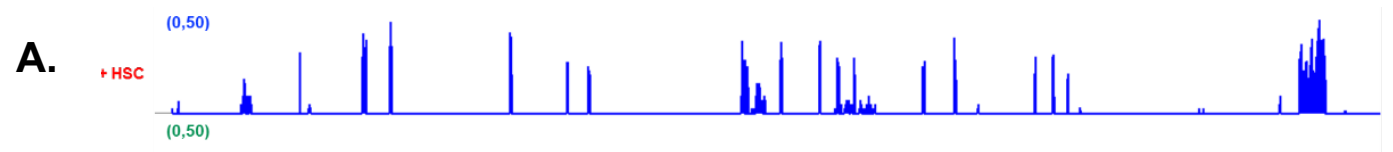

LEPR.HSC

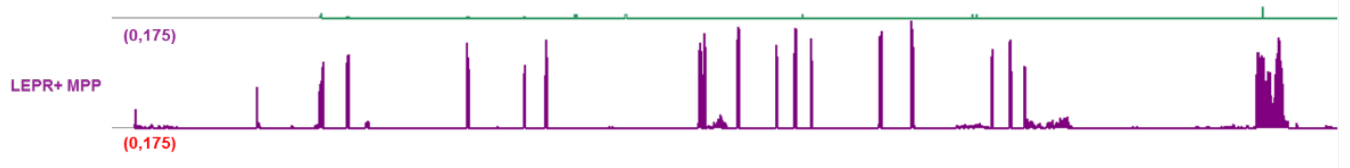

LEPR-MPP

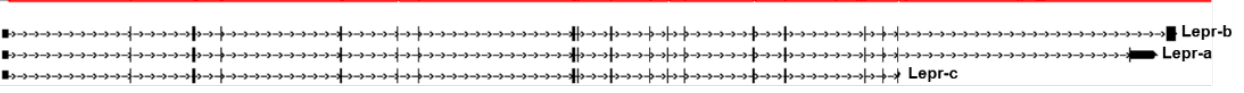

B.

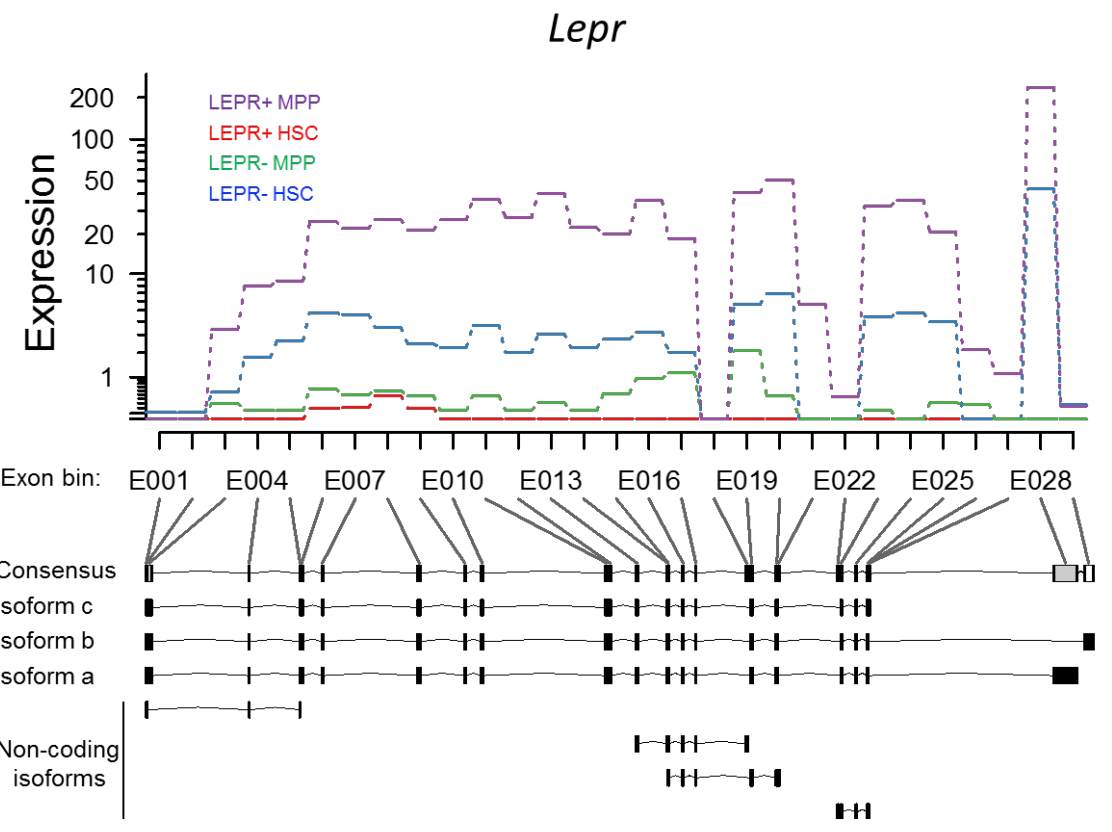

This was done with help from Dr. James Ropa. 
Figure 3.14 LEPR ${ }^{+}$HSC/MPP expressed predominantly the short isoforms Lepr-a and Lepr-c but not the long isoform Lepr-b.

(A). Normalized RNA-seq tracks demonstrate that there are many reads mapped to the Lepr exon 19 associated with Lepr isoform a, and very few mapped to the Lepr exon 19 associated with Lepr isoform b in murine HSCs and MPPs.

(B). RNA-seq was reanalyzed by dividing exons into counting bins using unique annotated transcripts, and reads were assigned to exon bins. Exon bin 28, which contains Lepr exon 19 that is included in the Lepr transcript that codes isoform a is highly represented in the RNA-seq, while Lepr exon 19 that is included in the Lepr transcript that encodes isoform $b$ is expressed to a very low degree at the mRNA level. 
To further gain insight into the molecular pathways that characterized each cell population, we performed gene set analysis by fast gene set enrichment analysis (FGSEA). The results revealed that LEPR+HSCs were enriched for Type-I Interferon and Interferon-gamma (IFN- - ) response pathways, whereas LEPRHSCs were enriched for genes involved in mitochondrial membrane protein and respiratory electron transport chain with Normalized Enrichment Scores of 2 or higher (Figure 3.15A, Ci-ii respectively). LEPR-MPP cells were enriched for DNA replication genes, while LEPR ${ }^{+}$MPP cells were enriched for extracellular matrix and structure gene sets (Figure 3.15B). 
A.

FGSEA for HSCS

(MSigDB Collection 5)

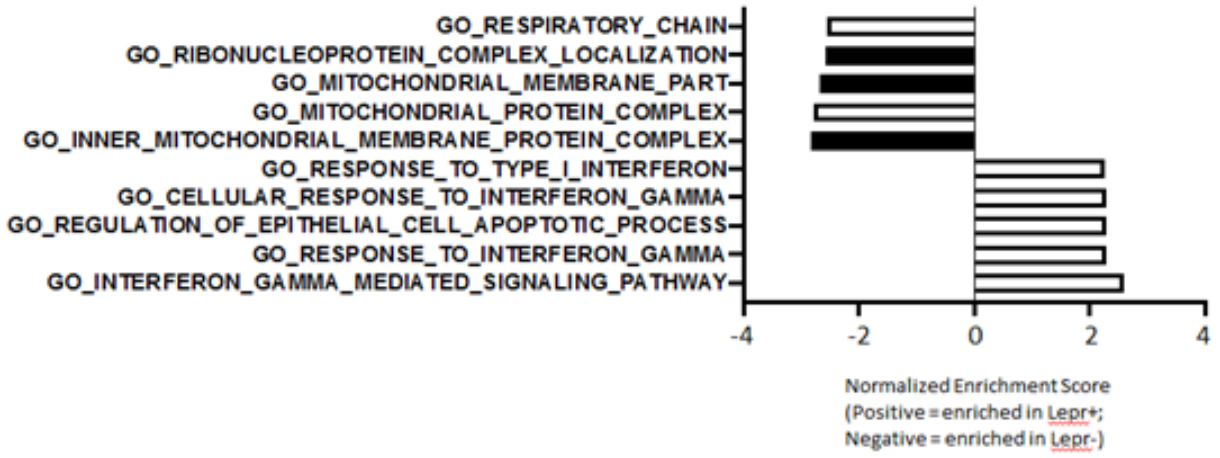

B.

FGSEA for MPPs (MSigDB Collection 5)

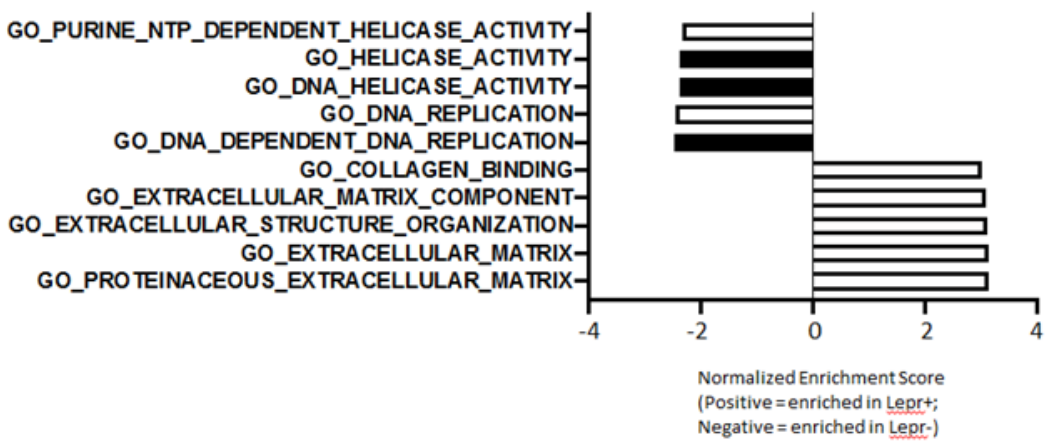

Ci. Cellular Response to IFN $\gamma(\mathrm{GO})$

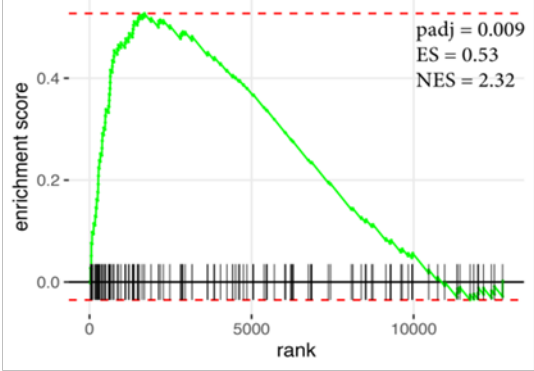

Cii. Mitochondrial Protein Complex (GO)

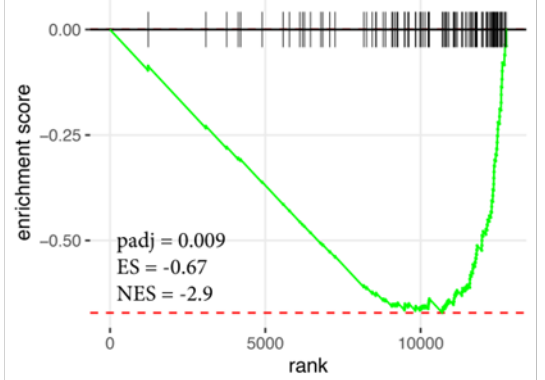

This was done with help from Dr. James Ropa. 
Figure 3.15 LEPR ${ }^{+} H S C s$ were characterized by proinflammatory transcriptomic pathways, whereas LEPR-HSCs were enriched for genes involved in mitochondrial membrane protein and respiratory electron transport chain.

LEPR ${ }^{+} H S C$ S/MPPs and LEPR-HSCs/MPPs were sorted from freshly isolated BM from C57BL6/J directly into 2- $\beta$ mercaptoethanol-supplemented lysis buffer and subjected to bulk RNA-sequencing ( $n=5$ biological replicates/population). A positive enrichment score indicates the gene program is enriched in $\mathrm{LEPR}^{+}$cells and vice versa.

(A). Gene set analysis showed enriched gene sets of molecular functions and biological processes in LEPR+HSCs using the R package "FGSEA" (B). Gene set analysis showed enriched gene sets of molecular functions and biological processes in LEPR ${ }^{+}$MPPs using the $R$ package "FGSEA" (Ci-ii). FGSEA plots for top hit pathways of LEPR+HSCs and LEPR-HSCs respectively. 
In addition, the top more highly expressed candidates in LEPR ${ }^{+} H S C$ s gene expression profile were enriched with genes involved in pro-inflammatory immune responses and regulatory processes including Relb and Rel, which encode subunits of the NF-KB complex (Figure 3.13B). This was particularly intriguing because both IFN- $y$ and NF-KB signaling pathways are characterized as being critical for HSC emergence in the aorta-gonad-mesonephros (AGM) during embryonic development (Espin-Palazon et al., 2014; Espin-Palazon \& Traver, 2016; Sawamiphak, Kontarakis, \& Stainier, 2014; Zhao et al., 2012). It has been demonstrated by others that deletion of NF-kB subunit p65/RelA led to impairments in HSC long-term engraftment (Stein \& Baldwin, 2013). In fact, further gene set analyses showed that LEPR+HSCs were enriched for genes associated with long-term HSCs, whereas LEPR-HSCs were enriched for an intermediate progenitor gene profile (Figure 3.16A, B respectively). 

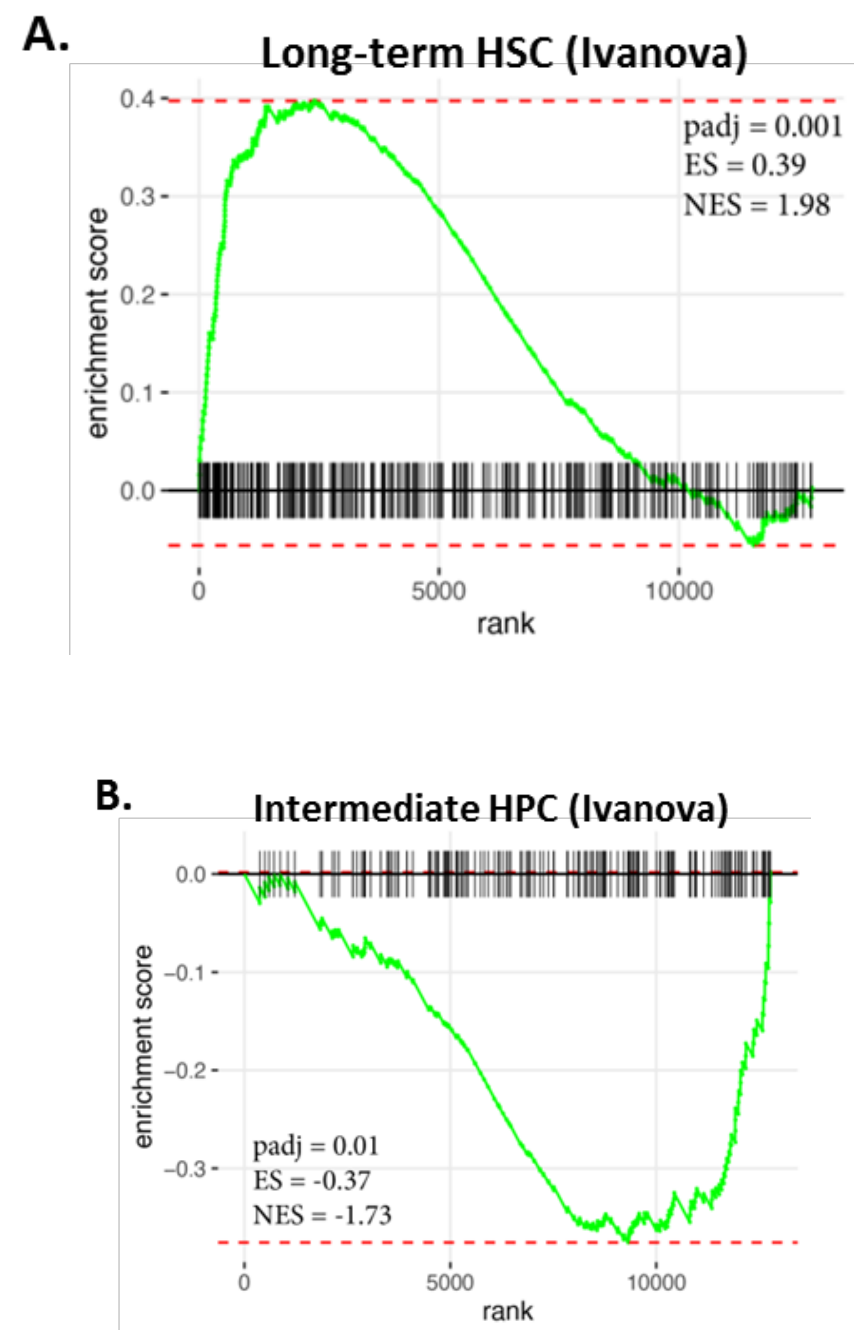

This was done with help from Dr. James Ropa. 
Figure 3.16 LEPR ${ }^{+}$HSCs were enriched for genes associated with long-term HSCs, whereas LEPR-HSCs were enriched for an intermediate progenitor gene profile.

LEPR ${ }^{+} H S C$ S/MPPs and LEPR-HSCs/MPPs were sorted from freshly isolated BM from C57BL6/J directly into 2- $\beta$ mercaptoethanol-supplemented lysis buffer and subjected to bulk RNA-sequencing ( $n=5$ biological replicates/population). A positive enrichment score indicates the gene program is enriched in $\mathrm{LEPR}^{+}$cells and vice versa.

(A-B). Additional FGSEA plots for gene sets associated with LT-HSC and progenitors as compared to LEPR ${ }^{+} H S C s$ and LEPR-HSCs profiles respectively. 


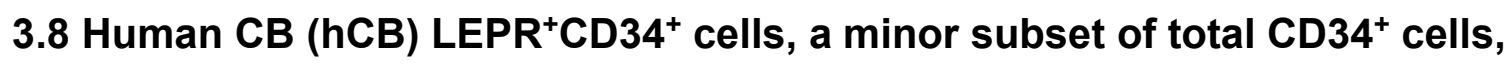
were more highly enriched for phenotypically defined HSCs and showed a trend to enhanced engraftment compared to LEPR-CD $34^{+}$cells in NSG mice Using FACS analyses, hCB CD34 ${ }^{+}$cells can be subdivided into CD34 dim (CD34 ${ }^{\mathrm{d}}$ ) and CD34 $4^{\text {bright }}\left(C D 34^{\text {bri }}\right)$. Interestingly most CD34 ${ }^{\text {bri }}$ were LEPR-expressing cells, which formed a distinct smaller subset out of the total $\mathrm{CD} 34^{+}$cell population (Figure 3.17A). To determine fractions of human phenotypic HSCs (pHSCs) and MPPs (pMPPs) within each population (LEPR ${ }^{+} \mathrm{CD} 34^{+}$vs. LEPR-CD34 $\left.4^{+}\right)$, I used a gating strategy as shown in Figure 3.17A. Consistent with mouse data for LEPR ${ }^{+} L S K$ vs. LEPR-LSK cells, hCB LEPR ${ }^{+} C D 34^{+}$cells contained significantly higher fractions of human phenotypically identified HSCs (defined as $\mathrm{CD}^{+}{ }^{+} \mathrm{CD} 38{ }^{-C D} 45 \mathrm{RA}^{-} \mathrm{CD} 90^{+}$) (Figure 3.17Bi), but a lower fraction of MPPs (defined as $\mathrm{CD}^{+} 4^{+} \mathrm{CD} 38^{-} \mathrm{CD} 45 \mathrm{RA}^{-} \mathrm{CD} 90^{-}$) (Figure 3.17 Bii). 


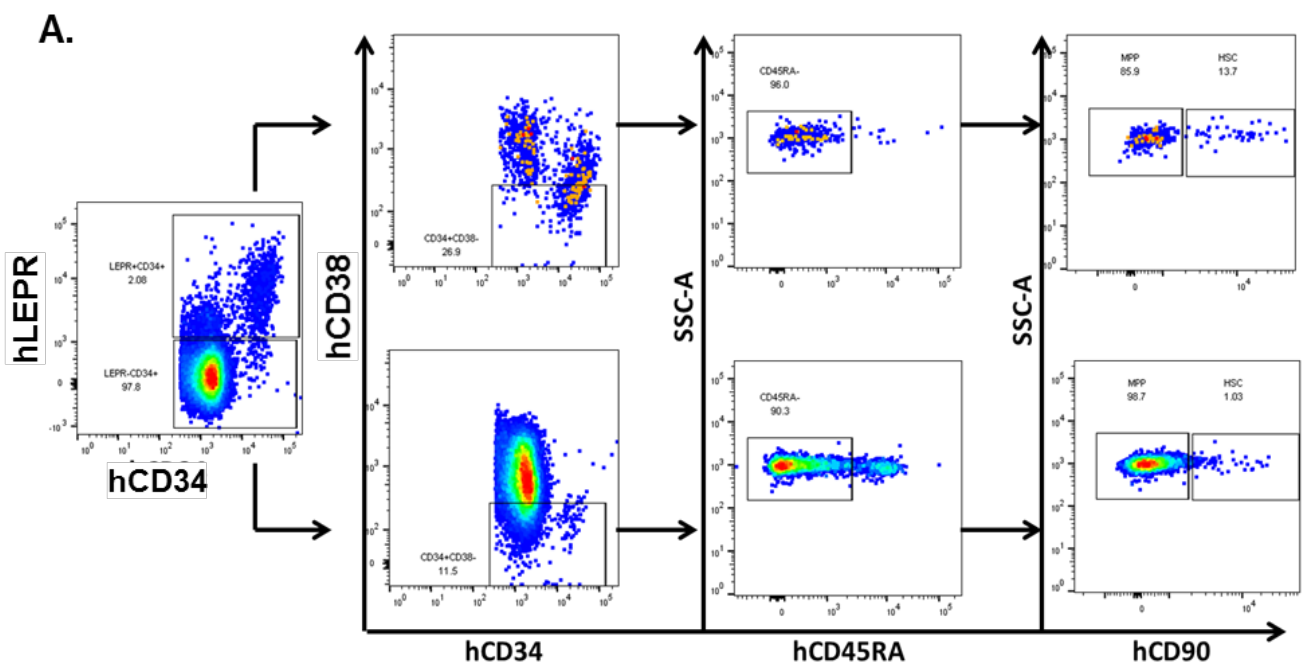

Bi.
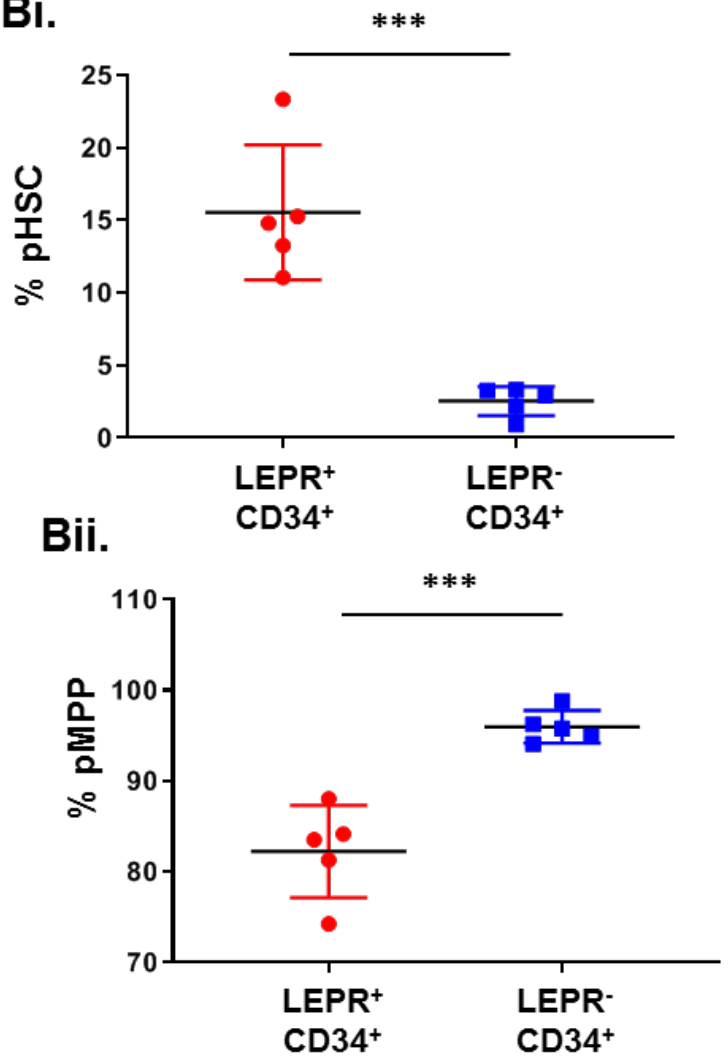

pHSC: $\mathrm{CD} 34^{+} \mathrm{CD} 38^{-} \mathrm{CD} 45 \mathrm{RA}^{-} \mathrm{CD} 90^{+} \quad$ pMPP: $\mathrm{CD} 34^{+} \mathrm{CD} 38^{-} \mathrm{CD}^{+} 4 \mathrm{RA}^{-} \mathrm{CD} 90^{-}$ 
Figure 3.17 Human CB (hCB) LEPR ${ }^{+} \mathrm{CD} 34^{+}$cells, a minor subset of total CD34 ${ }^{+}$cells, were more highly enriched for phenotypically defined HSCs. Using FACS analysis, freshly enriched hCB CD34+ cells were phenotyped for LEPR expression in human HSCs (defined as CD34 ${ }^{+}$CD38-CD45RA-CD90 ${ }^{+}$) and MPPs (defined as CD34+CD38-CD45RA-CD90-).

(A). Representative gating strategy to determine fractions of human phenotypically identified HSCs and MPPs within each population $\left(\mathrm{LEPR}^{+} \mathrm{CD} 34^{+}\right.$vs. LEPR-CD34 $\left.{ }^{+}\right)$

(Bi-ii). Fractions of human pHSCs (Bi) and pMPP (Bii) within each population $\left(\mathrm{LEPR}^{+} \mathrm{CD} 34^{+}\right.$vs. LEPR-CD34 ${ }^{+}$respectively $)(\mathrm{N}=5)$

Data are mean $\pm S D$. ${ }^{*} p<0.05,{ }^{* *} p<0.01,{ }^{* * *} p<0.001,{ }^{* * *} p<0.0001$ using Student's $\mathrm{t}$ test in (Bi-ii). 
To compare the differentiation potential of subsets of $\mathrm{CD}_{3} 4^{+}$cells in vitro, I plated equal numbers of freshly sorted LEPR ${ }^{+} C D 34^{\text {bri }}$, LEPR ${ }^{+} C D 34^{d}$ and LEPR-CD34 ${ }^{d}$ in a CFU assay. Numbers of colonies were determined after 14 days in culture. Interestingly, LEPR ${ }^{+} \mathrm{CD} 34^{\text {bri }}$ cells had the lowest absolute numbers of both CFUGM and CFU-GEMM colony-forming cells in comparison to the other two groups. There were no significant differences between LEPR ${ }^{+} C D 34^{d}$ and LEPR-CD34 ${ }^{d}$ groups (Figure 3.18Ai-ii).

To assess the engrafting potential of LEPR ${ }^{+} \mathrm{CD} 34^{+}$as compared to LEPR-CD34 ${ }^{+}$ cells, we performed a limiting dilution assay in which equal numbers of each population were sorted from freshly isolated $\mathrm{hCB} C \mathrm{CD} 34^{+}$-enriched cells and i.v. injected into sublethally-irradiated immune deficient NOD/SCID IL-2 receptor gamma chain null (NSG) mice. SCID-repopulating cells (SRCs) were calculated using Poisson statistical analysis (Figure 3.18Bi). LEPR ${ }^{+} \mathrm{CD} 34^{+}$cells were enriched with a 2.9 fold difference of SRCs compared to LEPR-CD $34^{+}$cells in the highest dose $(p=0.1$; Figure 3.18Bii). Consistent with the mouse data, using percentages of human $\mathrm{CD}^{+} \mathrm{T}$ cells, $\mathrm{CD} 19^{+} \mathrm{B}$ cells and $\mathrm{CD} 33^{+}$myeloid cells the myeloid/lymphoid ratio of engrafted $\mathrm{CD} 34^{+}$cells calculated was similar in all groups (Figure 3.18C). 


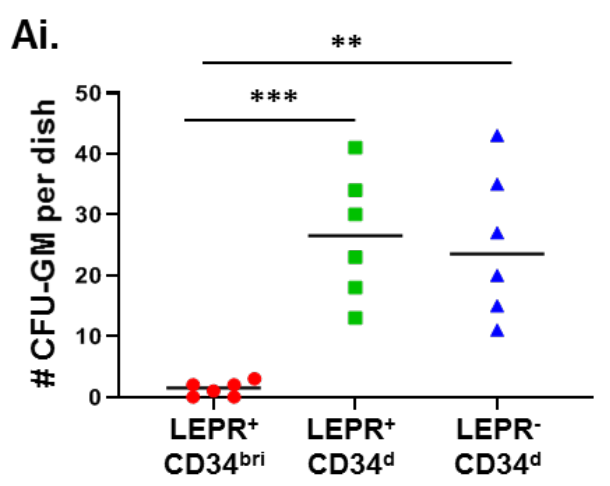

Aii.
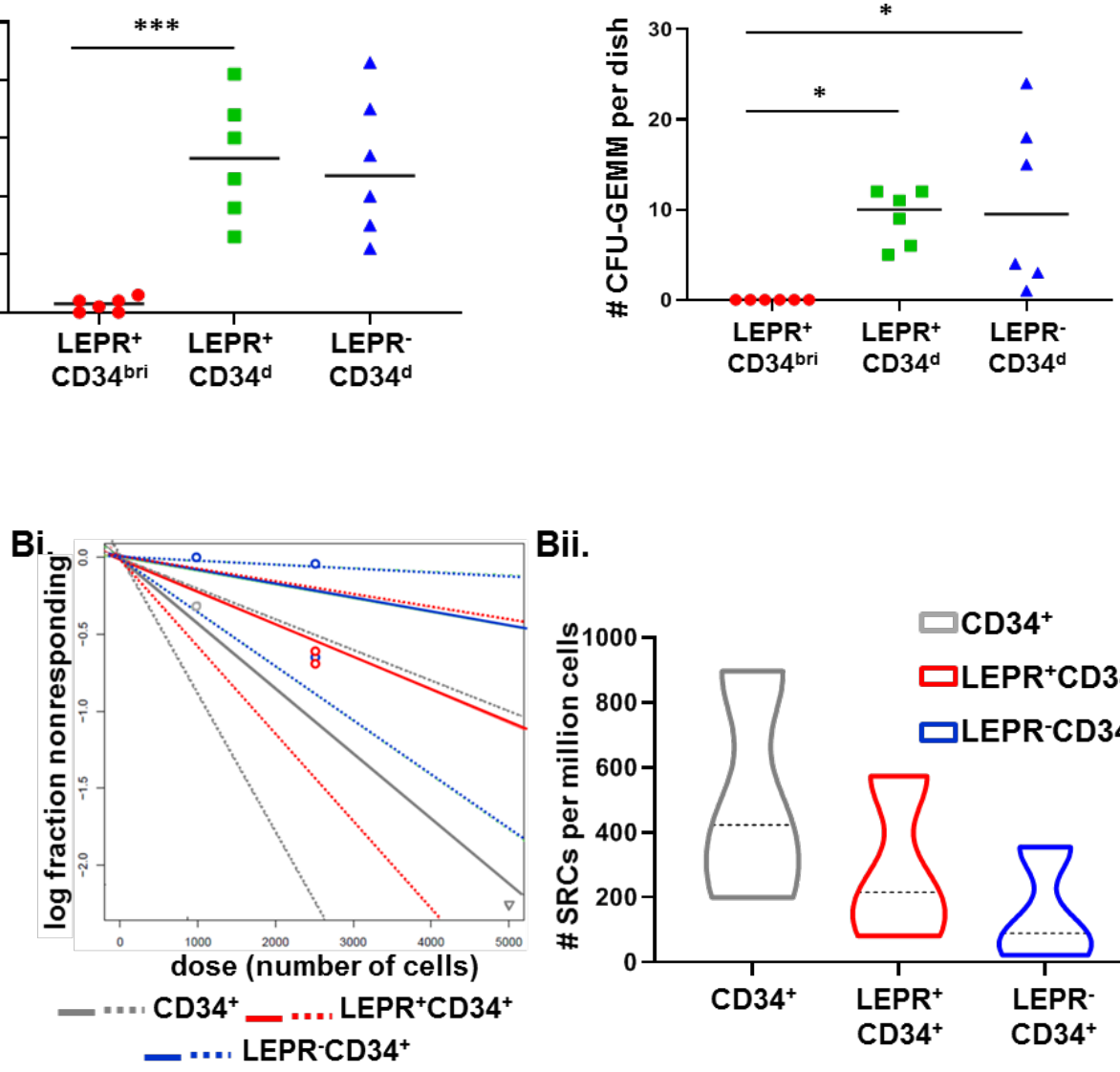

Bii.

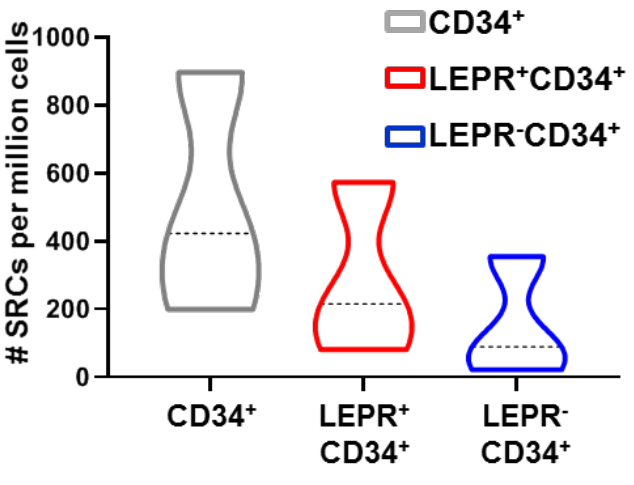

c.

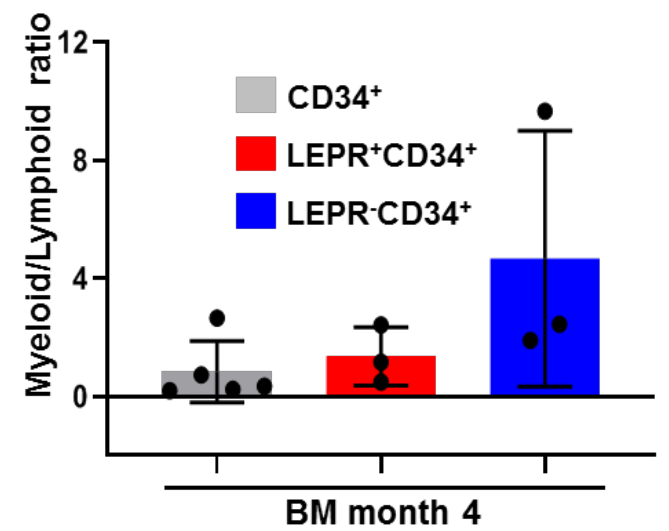


Figure 3.18 hCB LEPR ${ }^{+}$CD $34^{+}$cells showed a trend to enhanced engraftment compared to LEPR-CD $34^{+}$cells in NSG mice.

(Ai-ii). Freshly sorted hCB LEPR ${ }^{+}$CD34bri, LEPR ${ }^{+}$CD34dim and LEPR-CD34dim cells were assessed for colony formation capacity in CFU assay.

Absolute numbers of CFU-GM (Ai) and CFU-GEMM (Aii) colonies scored 14 days after plating equal numbers of LEPR ${ }^{+} C D 34^{\text {bri }}$, LEPR ${ }^{+}$CD34 ${ }^{\text {dim }}$ and LEPR ${ }^{-}$ CD34 ${ }^{\text {dim }}$ in semisolid methylcellulose supplemented with GFs and cytokines. $(\mathrm{N}=$ 2 independently repeated experiments)

(Bi). Different doses $(1000,2500,5000)$ of freshly sorted total CD34 ${ }^{+}$, $\mathrm{LEPR}^{+} \mathrm{CD} 34^{+}$or LEPR-CD34 ${ }^{+}$cells were i.v. injected at equal numbers into sublethally irradiated immunocompromised NSG mice in a limiting dilution assay. Poisson statistical analysis from the limiting dilution analysis. Solid lines represent the best-fit linear model for each data set. Dotted lines represent $95 \%$ confidence intervals. Symbols represent the percentage of negative mice for each dose of cells. Plot was modified from the original for better clarity. (Bii). \# of SRCs per one million injected cells calculated from (Bi) Line indicate median.

(C). Myeloid/lymphoid ratio was calculated using percentages of human CD33 ${ }^{+}$ myeloid, $\mathrm{CD}^{+} \mathrm{T}$ and $\mathrm{CD} 19^{+} \mathrm{B}$ cells with no statistical differences.

Data are mean $\pm S D .{ }^{*} p<0.05,{ }^{* *} p<0.01,{ }^{* * *} p<0.001,{ }^{* * * *} p<0.0001$ using Ordinary One-way ANOVA followed with post-hoc Tukey's multiple comparison test in (Ai-ii, C). Poisson statistical analysis in $(\mathrm{Bi}) \mathrm{p}=0.1$. 


\subsection{Phenotypic characterizations of LEPR ${ }^{+}$hematopoietic stem and progenitor cells in middle-aged mice as compared to LEPR' cells}

The metabolic activities of a cell are adaptively regulated by systemic signals that reflect the nutritional status of an organism throughout its life (Shapira \&

Christofk, 2020; Y. P. Wang \& Lei, 2018). This is particularly crucial in the case of HSCs as they are rare, and they have to maintain the integrity of an entire organ system. One way that the body communicates nutritional cues to HSCs is via systemic hormonal regulations. Indeed, various metabolic hormones have been well-documented to influence hematopoiesis, and aging can significantly alter these metabolic messengers hence indirectly affects HSC functional behaviors (Stewart et al., 2014; Xia et al., 2015; Zhang et al., 2017).

As an adipokine, leptin is recognized to have a broad spectrum effects on numbers and functions of different immune cells under homeostasis (La Cava \& Matarese, 2004). Aging is known to be associated with multiple immune dysregulations including declined adaptive immune responses (Ongradi \& Kovesdi, 2010). Recently, leptin has been demonstrated to induce gene expression of p16, a marker of cellular immunosenescence in human B cells from young lean adults. These cells also exhibited lowered class switching activity and ability to produce influenza-specific IgG (Frasca \& Blomberg, 2017; Frasca, Diaz, Romero, \& Blomberg, 2020). Unfortunately, the study was limited to in vitro treatment only and did not provide full mechanistic insights. In line with this finding, Gupta et al. demonstrated that leptin induced significantly higher 
levels of IL-10, TNF- $\alpha$, and IL6 from aged human B cells as compared to young controls, and this effect was mediated through the STAT3 signaling pathway downstream of leptin receptor activation (S. Gupta, Agrawal, \& Gollapudi, 2013). Another group reported that sustainably higher leptin levels were found in LPStreated old (24 month) rats compared to young ( 2 month) animals, and the old rats showed delayed but longer febrile response. Elevated leptin concentrations were accompanied by increased levels of pro-inflammatory cytokines including IL-6 and IL-1Ra; however, it was not determined whether the increase in leptin level was causative and how (Koenig et al., 2014). Although leptin signaling was consistently reported to be altered in aged animals, more rigorously designed studies are needed to help us understand how this well-known proinflammatory neuroendocrine adipokine may play any roles in aging-associated immunological changes (Filippi \& Lam, 2014; Gabriely, Ma, Yang, Rossetti, \& Barzilai, 2002).

In addition, it has been demonstrated that BM adipose tissue possessed brown fat properties that declined in old or diabetic mice (Krings et al., 2012). Interestingly, Ambrosi et al. demonstrated that during the process of aging or in obesity MSCs preferentially differentiated into adipocytes, which impaired hematopoietic recovery (Ambrosi et al., 2017). Since BM adipocytes could be a potential source of leptin, it is important to know how BM adipocyte-derived leptin can indirectly alter hematopoiesis as the animal ages. Given that LEPR (OB-R) was also reported to be expressed by different types of both myeloid and lymphoid leukemic cells (Z. Lu et al., 2017; Nakao et al., 1998; Ozturk et al., 
2012; Wex et al., 2002), it will be intriguing and important to determine whether aging has a differential selection pressure on LEPR ${ }^{+} H S C s$ as compared to the rest of total BM HSCs particularly in the context of clonal hematopoiesis.

To assess how LEPR-expressing HSC and HPC populations may alter differentially from LEPR- cells with aging, I first performed FACS phenotypic analyses (Figure 3.19). Interestingly, results revealed that fractions of the more immature populations such as long-term HSCs (LT-HSC defined as LSKFLT3CD34-) (Figure 3.20Ai), short-term HSCs (ST-HSC defined as LSKFLT3-CD34+) (Figure 3.20Aii, ), and multipotent progenitor (MPP defined as LSKFLT3 ${ }^{+} \mathrm{CD} 34^{+}$) (Figure 3.21A) expanded significantly with aging as fractions of LEPR-cells reduced. Consistent with this, fractions of long-term SLAM HSCs (SLAM HSCLT defined as LSKFLT3-CD150+CD48-) (Figure 3.20Bi), the lymphoid-biased MPP4 (LSKFLT3 $^{+}$CD150-CD48 ${ }^{+}$) (Figure 3.21Bi), the myeloid-biased MPP2 (LSKFLT3-

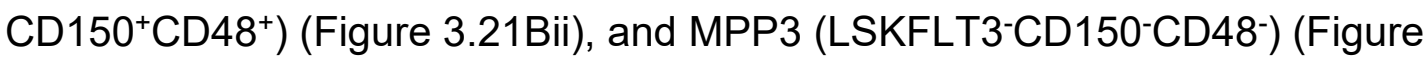
3.21Biii), all significantly increased as the mice aged. However, short-term SLAM HSCs (SLAM HSC ${ }^{\text {ST }}$ defined as LSKFLT3-CD150-CD48-') remained similar in proportions as compared to LEPR- cells in young vs. older mice (Figure 3.20Bii). On the same line, the more mature LEPR-expressing progenitor populations including CMP, GMP and MEP remained significantly lower in proportions compared to LEPR- cells in young vs. middle-aged mice (Figure 3.22A-C respectively). Altogether, the data suggested that aging affected LEPR- 
expressing stem and more immature multipotent progenitor cell groups to a greater extent than the more differentiated progenitor cells. 
Figure 3.19 Representative plots of HSCs in middle-aged mice in comparison to young mice.

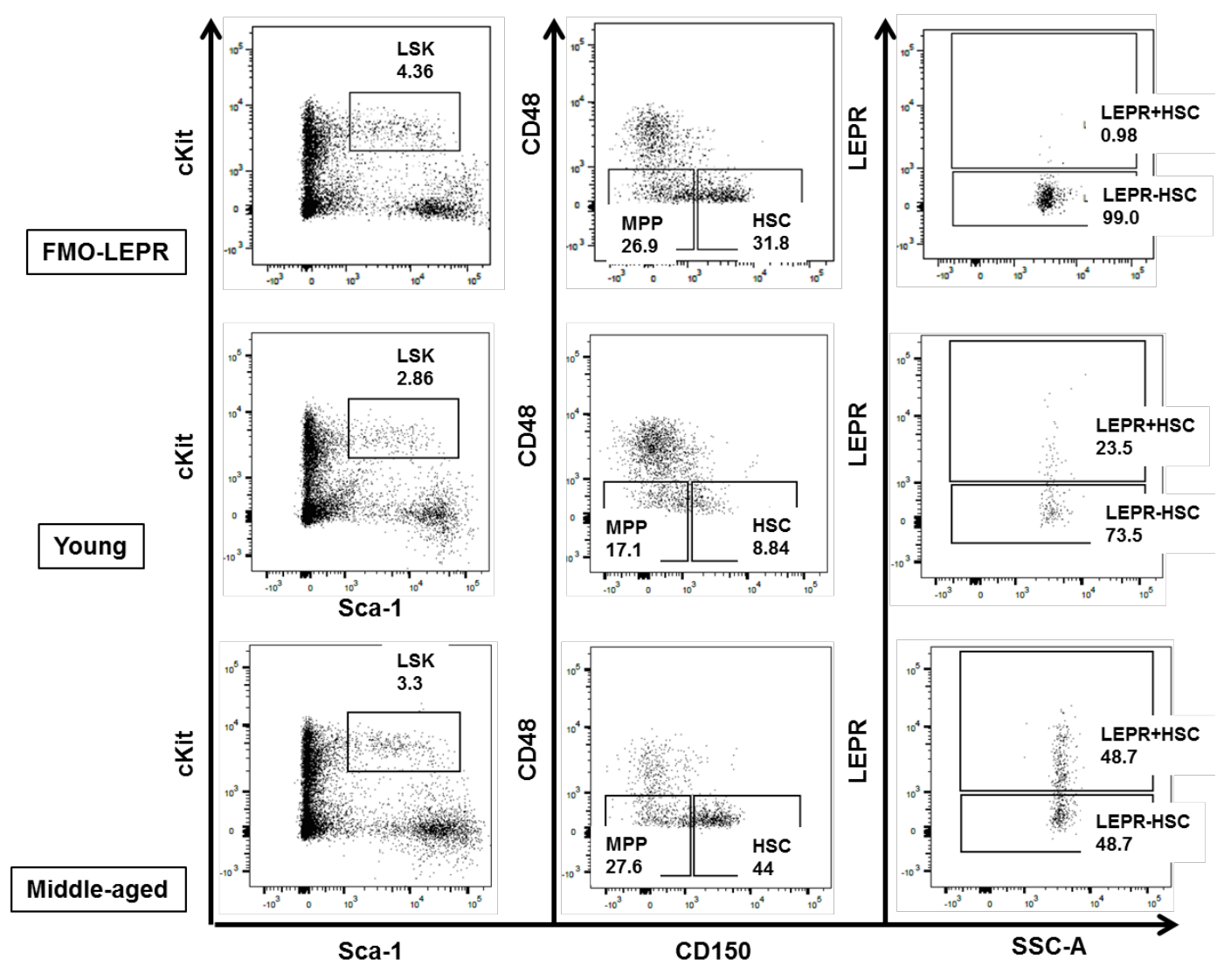



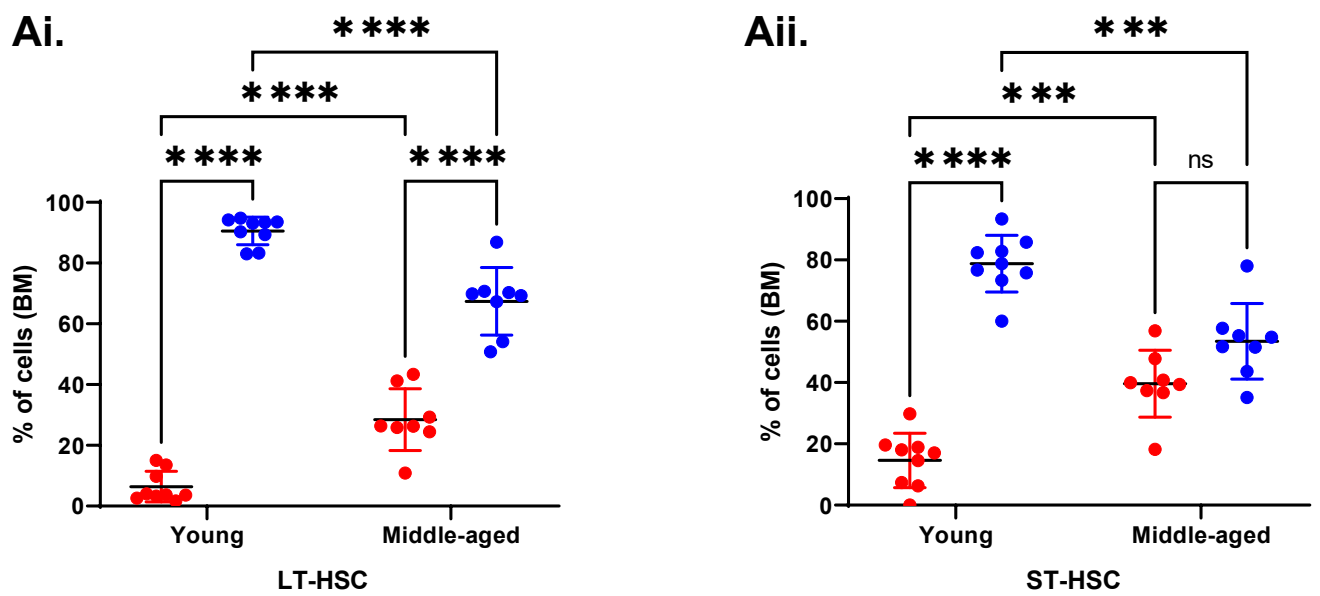

\section{- LEPR $^{+}$ \\ - LEPR $^{-}$}
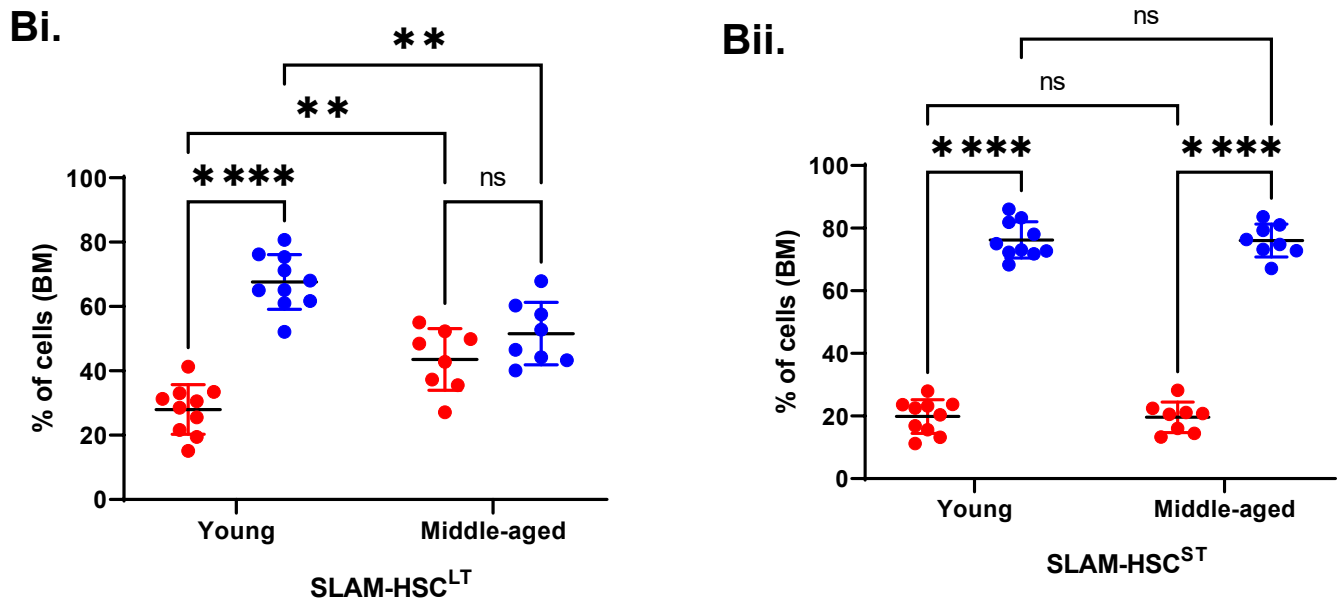
Figure 3.20 With age, fractions of long-term LEPR ${ }^{+}$SLAM HSCs expanded over LEPR- SLAM HSCs

Freshly isolated BM from either young adult or middle-aged C57BL6/J mice $(\mathrm{n}=$ 8-10/group) were immunophenotypically analyzed for long-term and short-term HSCs.

(Ai-ii). Percentages of LEPR ${ }^{+}$SLAM HSC ${ }^{L T}$ and LEPR+ SLAM HSC ${ }^{S T}$ as compared to LEPR- cells in young vs. middle-aged mice.

(B) Percentages of LEPR ${ }^{+}$LT-HSC and LEPR+ ST-HSC as compared to LEPRcells in young vs. middle-aged mice.

Data are mean \pm SD. ${ }^{*} p<0.05,{ }^{* *} p<0.01,{ }^{* * *} p<0.001,{ }^{* * * *} p<0.0001$ using Ordinary Two-way ANOVA followed with post-hoc Tukey's multiple comparison test. $\mathrm{N}=2$. 

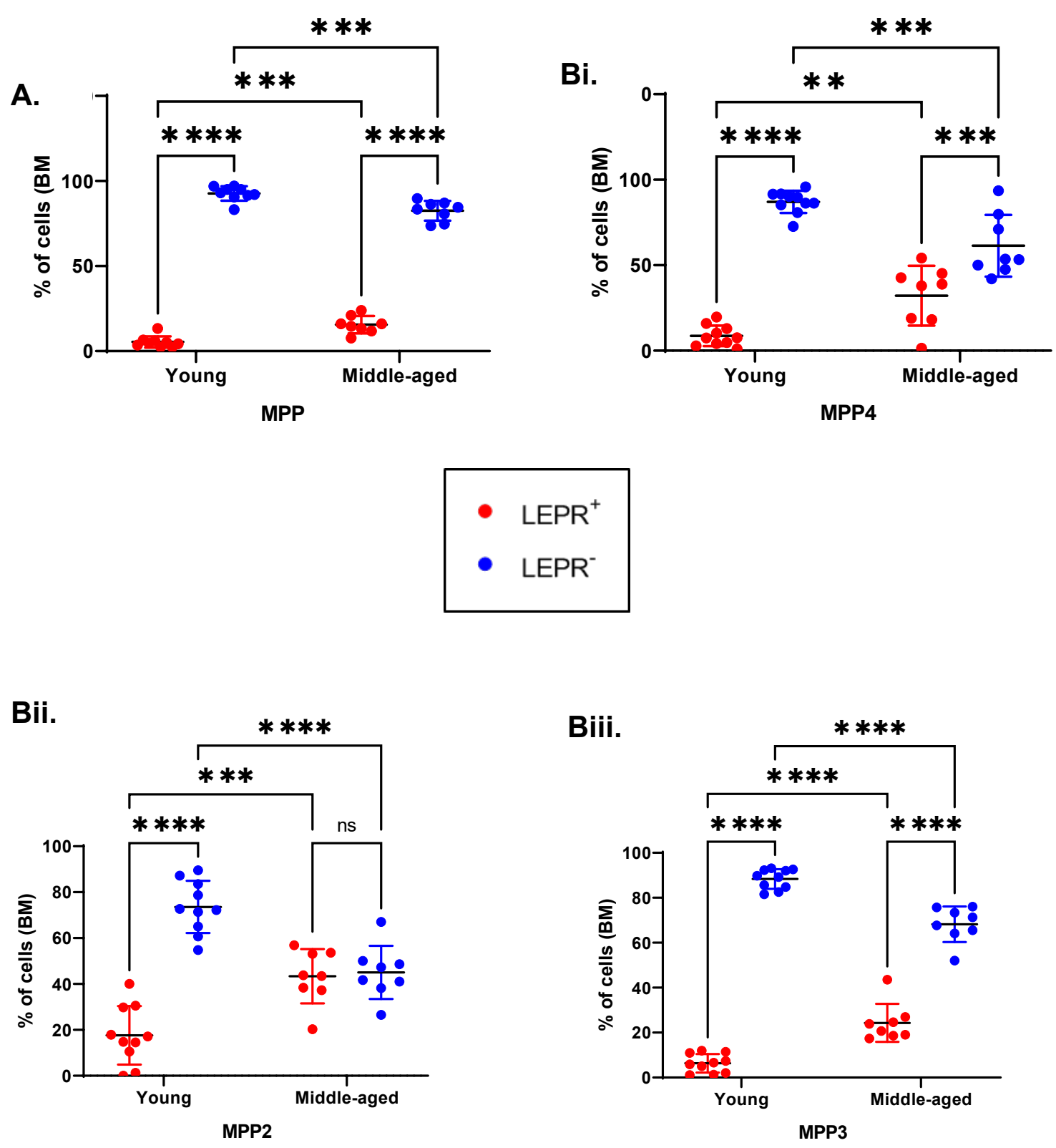
Figure 3.21 With age, fractions of total MPP and different lineage-biased MPP subsets expanded over LEPR'populations.

Freshly isolated BM from either young adult or middle-aged C57BL6/J mice $(n=$ 8-10/group) were immunophenotypically analyzed for total and lineage-biased MPP subsets.

(A). Percentages of LEPR ${ }^{+}$total MPP as compared to LEPR cells in young vs. middle-aged mice.

(B) Percentages of LEPR ${ }^{+}$MPP2, MPP3, and MPP4 as compared to LEPR- cells in young vs. middle-aged mice.

Data are mean $\pm S D$. ${ }^{*} p<0.05,{ }^{* *} p<0.01,{ }^{* * *} p<0.001,{ }^{* * * *} p<0.0001$ using Ordinary Two-way ANOVA followed with post-hoc Tukey's multiple comparison test. $\mathrm{N}=2$. 
A.

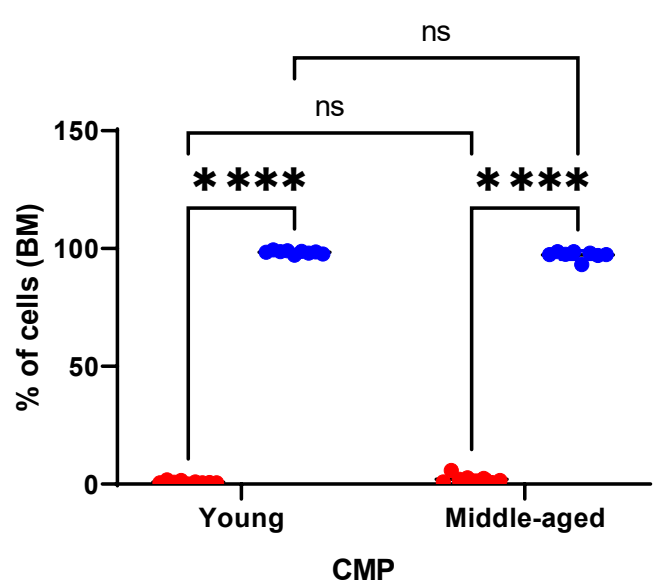

B.

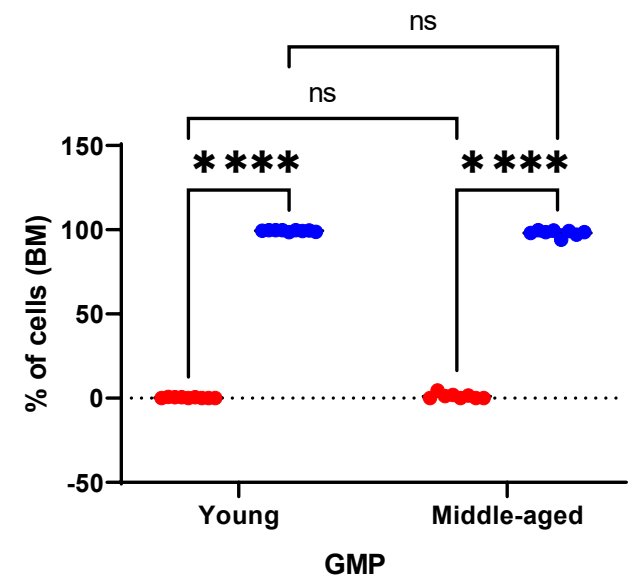

c.

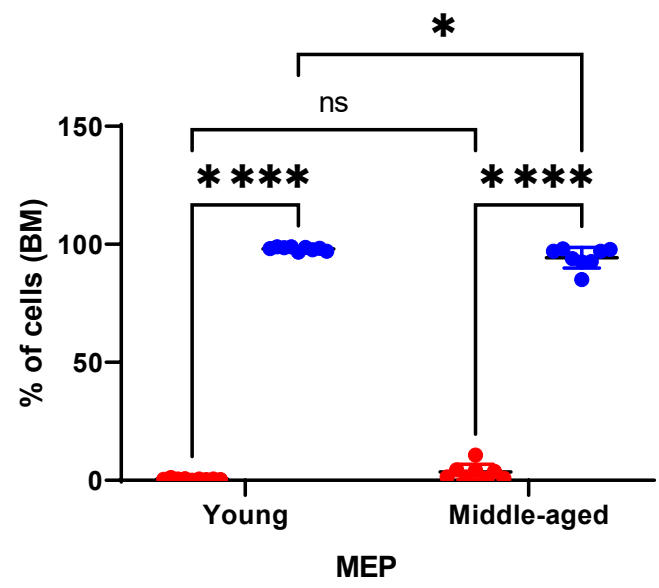


Figure 3.22 Unlike stem cells, LEPR ${ }^{+}$progenitor populations did not expand with aging and remained as low as in young BM.

Freshly isolated BM from either young adult or middle-aged C57BL6/J mice $(n=$ 8-10/group) were immunophenotypically analyzed LEPR and lineage-specific progenitor cells.

(A). Percentages of LEPR ${ }^{+}$CMP as compared to LEPR- cells in young vs. middle-aged mice.

(B) Percentages of LEPR ${ }^{+}$GMP as compared to LEPR- cells in young vs. middleaged mice.

(B) Percentages of LEPR ${ }^{+}$MEP as compared to LEPR- cells in young vs. middleaged mice.

Data are mean $\pm S D .{ }^{*} p<0.05,{ }^{* *} p<0.01,{ }^{* * *} p<0.001,{ }^{* * *} p<0.0001$ using Ordinary Two-way ANOVA followed with post-hoc Tukey's multiple comparison test. $\mathrm{N}=2$. 


\section{CHAPTER FOUR: DISCUSSION AND FUTURE DIRECTIONS}

\subsection{Discussion}

HCT is widely used to treat, and in certain cases to cure patients with hematologic malignancies or other blood and immune disorders. Each method of HCT has its own advantages and disadvantages as aforementioned.

Remarkably, CB transplantation has multiple important advantages over traditional allogeneic HCT including but not limited to reduced risks of lifethreatening complications such as graft vs. host disease (Ballen, Gluckman, \& Broxmeyer, 2013; Mayani, Wagner, \& Broxmeyer, 2020). Unfortunately, one of the major impediments hindering advancements of the field is the insufficient number of functional and immune-compatible HSCs for larger pediatric and adult patients (Ballen et al., 2013; Mayani et al., 2020; Talib \& Shepard, 2020). Efforts to increase the number of engrafting HSCs are crucial for better clinical outcomes. For this reason, a reliable phenotyping marker for HSCs for ex vivo expansion that is well-correlated with their engrafting function in vivo is practically needed.

Consistent with others' findings, I did not find any phenotypic changes in murine BM hematopoietic compositions of germ-line Lepr/- mice as compared to WT. Our data demonstrated that LEPR ${ }^{+} L S K$ cells although representing a much smaller percentage compared to LEPR-LSK cells, were significantly enriched with higher numbers of long-term CRUs, as demonstrated in limiting dilution assay, 
and possessed extensive and robust self-renewal capacity in secondary transplants. Importantly, further functional characterizations revealed LEPR differentiating SLAM HSCs (LSKCD150+CD48-) into two functionally distinct populations of HSCs. LEPR ${ }^{+}$SLAM HSCs being a very minor subset of total SLAM HSCs in the mouse BM, exhibited significantly higher repopulating potential and self-renewing capacity in comparison to LEPR- SLAM HSCs. To gain insight into the transcriptomic regulations that may be responsible for the functional properties of LEPR-expressing stem and progenitor cells, bulk-RNA seq was performed and showed that mouse BM LEPR ${ }^{+}$SLAM HSCs were characterized by a unique molecular network - a proinflammatory transcriptomic profile and pathways that are crucial for embryonic HSC development. Our data thus suggested that LEPR ${ }^{+} H S C$ s represent a small but highly potent subset of adult HSCs that retained an embryonic-like molecular signature with robust longterm primary and secondary repopulating potential. Considering the current knowledge that phenotype may not recapitulate function for HSCs (Chen et al., 2019), our work helps to identify a reliable marker for highly repopulating and self-renewing long-term adult BM HSCs. Though still preliminary and limited in scope, our extended data in older mice revealed that as mice aged (even as young as $13-16$ months old or middle-age) fractions of LEPR-expressing HSCs and MPPs expanded significantly while fractions of LEPR' cells decreased concomitantly. 
Consistent with mouse data, our studies of LEPR in human umbilical cord blood CD $34^{+}$cells illustrated that only a small portion of $\mathrm{hCB} C D 34^{+}$cells expressed LEPR. In a similar comparison to murine LEPR ${ }^{+}$LSK cells, LEPR ${ }^{+}$CD $34^{+}$cells were highly enriched for phenotypically-defined human HSCs, and functionally they engrafted better than LEPR-CD $34^{+}$cells in a limiting dilution assay transplant. Although the human engraftment studies did not meet statistical significance, because leptin and leptin receptor are highly conserved between mouse and human, our findings may have implications for the fields of both adult $\mathrm{BM}$ and $\mathrm{CB}$ transplantation. Table 4.1 summarizes the major findings from our study. 


\begin{tabular}{|c|c|}
\hline \multicolumn{2}{|r|}{ Table 4.1 Summary of major findings (Trinh et al., 2020) } \\
\hline Mouse & $\begin{array}{l}\text { - Only a very small percentage of BM hematopoietic cells } \\
\text { express LEPR } \\
\text { - Within different LEPR-expressing hematopoietic populations, } \\
\text { fractions of LEPR }{ }^{+} \text {stem cells are significantly higher than } \\
\text { LEPR+ progenitor cells } \\
\text { - } \text { LEPR }^{+} \text {LSK cells are significantly enriched for phenotypically } \\
\text { defined HSCs, MPPs and contain higher absolute numbers of } \\
\text { colony-forming progenitor cells and self-renewal CRUs } \\
\text { - LEPR+ SLAM HSCs engraft better and contain significantly } \\
\text { more CRUs than LEPR-SLAM HSCs } \\
\text { LEPR+HSCs share similar homing capacity, lineage output } \\
\text { and cycling status with LEPR-HSCs } \\
\text { Molecularly, LEPR }{ }^{+} H S C s \text { are characterized by a } \\
\text { proinflammatory transcriptome and more highly enriched for } \\
\text { genes associated with LT-HSCs, whereas LEPR-HSCs are } \\
\text { more highly enriched for progenitor-associated gene sets } \\
\text { expanded as compared to LEPR+HSCs }\end{array}$ \\
\hline hCB & $\begin{array}{l}\text { - } \mathrm{LEPR}^{+} \mathrm{CD} 34^{+} \text {cells represent a small subset out of total CD34 } \\
\text { cells }\end{array}$ \\
\hline
\end{tabular}




\begin{tabular}{|l|l|}
\hline$\bullet$ & LEPR $^{+}$CD $34^{+}$cells show a trend toward enhanced \\
engraftment as compared to LEPR-CD $34^{+}$cells
\end{tabular}


Beyond the scope of our study, the presence of LEPR in human leukemia cell lines and primary patient samples is repeatedly reported in the literature (Gorska et al., 2013; Konopleva et al., 1999; Z. Lu et al., 2017; Nakao et al., 1998; Ozturk et al., 2012). A recent study (Z. Lu et al., 2017) demonstrated that LEPR signaling mediated the protective effects of fasting on acute lymphoblastic leukemia (ALL), but not AML, cells. The study provided therapeutically relevant mechanisms. Using the activated Notch1 T-ALL model, the N-Myc B-ALL model and the MLL-AF9 AML model, it was found that fasting significantly suppressed $A L L$, but not $A M L$, development by promoting blast cell differentiation and hence prolonged leukemia-induced mouse survival. RNA-seq analyses of leukemic BALL cells revealed expression levels of multiple cytokine receptors upregulated by fasting including Lepr and its major downstream effector, the transcription factor STAT3. This study (Z. Lu et al., 2017) also pointed out that expression of LEPR was positively correlated to survival rates in patients with pre-B-ALL. It was confirmed that B-ALL, T-ALL and AML leukemic cells express the long isoform $O B-R b$; likewise, fasting did not upregulate Lepr in AML as it did in B-ALL and TALL cells. To investigate potential role(s) that LEPR signaling plays in ALL development, WT mice were transplanted with N-Myc-infected BM Lin- cells that either came from WT $\left(\operatorname{Lepr}^{+/+}\right)$or $\operatorname{Lepr}^{--}(d b / d b)$ mice. Recipient mice that received Lepr/- leukemia-transformed BM cells developed B-ALL at a significantly faster rate, had higher multi-organ leukemic burden and lower survival rate. The $L e p r^{--}$group had a higher percentage of $B 220^{+} \mathrm{CD} 43^{+}$ precursors and a lower percentage of differentiated $\mathrm{B}^{2} 20^{+} \operatorname{IgM}^{+}$cells, which 
suggested that LEPR signaling was critical for the differentiation of B-ALL cells. Similar results were observed in T-ALL but not in AML models ( $Z$. Lu et al., 2017). Furthermore, the investigators repeated these experiments, but recipient mice were fasted for $48 \mathrm{~h}$ upon attaining about $60 \%$ of leukemic-transformed $\mathrm{GFP}^{+}$cells in PB. Consistent with initial findings, fasting attenuated percentages of $\mathrm{GFP}^{+}$leukemic cells and increased percentages of differentiated $\operatorname{lgM}^{+}$cells in the WT $\left(\mathrm{Lepr}^{+/+}\right)$, but not in Lepr/-, group. This confirmed that the anti-leukemic effects of fasting were mediated through LEPR signaling (Z. Lu et al., 2017).

Mechanistically, LEPR overexpression in B-ALL cells led to upregulation of key transcription factors in B cell terminal differentiation including Xbp1 and Prdm1 (Z. Lu et al., 2017). Overexpression of Prdm1 blocked B-ALL development, and knockdown of Prdm1 inhibited the anti-leukemic effect of LEPR overexpression in B-ALL. In silico analyses of patient databases showed that LEPR signaling and its related genes were positively correlated with better outcome and survival in pre-B-ALL leukemia. It was also demonstrated that fasting negatively regulated B-ALL development in a xenograft model transplanted with human pre-B-ALL cell line NALM-6, and it did so by upregulating LEPR expression (Z. Lu et al., 2017).

A $\mathrm{Lep}^{-/}$mouse model $(\mathrm{Ob} / \mathrm{Ob})$ was used to investigate potential effects of obesity on development of AML from myeloid dysplastic syndrome (MDS) and its associated mortality rate (Kraakman et al., 2018). Using BM from NUP98HOXD13 transgenic mice, a model of MDS, compared to lean WT controls, 
obese $L e p^{-/}$recipient mice had significantly higher survival rates, and opposite to the researchers' hypothesis these mice did not have increased risk of AML transformation. While Lep/- recipient mice transplanted with NUP98-HOXD13 BM had more severe monocytosis, it was suggested that their expanded adipose tissue was capable of harboring more myeloid cells, and hence reduced their detrimental accumulation in other vital organs such as the liver (Kraakman et al., 2018). Even though the authors rationalized their reasons for excluding the potential effects of Lep knockout on their findings, it would have been of more interest if the phenotypes were consistent in a different model of obesity and NUP98-HOXD13 induced MDS. Furthermore, considering that obese individuals are at higher risk for leukemia development and obese children afflicted with leukemia have increased mortality rate (Ladas et al., 2016; X. Ma et al., 2009; Murphy et al., 2013; Weiss, Vogl, Berger, Stadtmauer, \& Lazarus, 2013), this study posed discrepancies between mice and humans, and thus requires future in-depth reevaluation.

In addition to being present on leukemic hematopoietic lineage cells, LEPRexpressing stromal cells have also been demonstrated to be involved in BM fibrosis of primary myelofibrosis (PMF), a subtype of myeloproliferative neoplasms (Decker et al., 2017). While LEPR ${ }^{+}$mesenchymal stromal cells have been studied more extensively in normal hematopoiesis, the malignancy transformation potential of these prominent niche cells is far less understood. It was demonstrated that these cells were functionally and molecularly altered 
during the progression of thrombopoietin (TPO)-overexpressing-induced PMF (Decker et al., 2017). Specifically, by using different genetic fate mapping models it was discovered that as the mice developed PMF, LEPR ${ }^{+}$stromal cells significantly expanded in numbers and increased production of collagen, which led to BM hematopoietic failure, fibrosis and extramedullary hematopoiesis. In line with this finding, gene expression analyses demonstrated that LEPR ${ }^{+}$stromal cells down-regulated HSC supporting growth factors including $\mathrm{Cxc} / 12$ and Scf while up-regulating genes involved in fibrotic conversion. Importantly, mechanistic studies pinpointed platelet-derived growth factor receptor alpha (PDGFR $\alpha$ ) as the key player in the pathogenesis of bone marrow fibrosis caused by LEPR ${ }^{+}$stromal cells. This was therapeutically relevant since imatinib, an inhibitor of different tyrokine kinases including PDGFRa, was shown to lessen fibrosis in this model of PMF (Decker et al., 2017). Even though the study focused on PMF, it has opened up a new area for future studies into microenvironmental niche cells in other hematological malignancies, which is arguably just as important as studying the leukemic cells themselves. 


\section{Figure 4.1}

\section{A. Steady state}

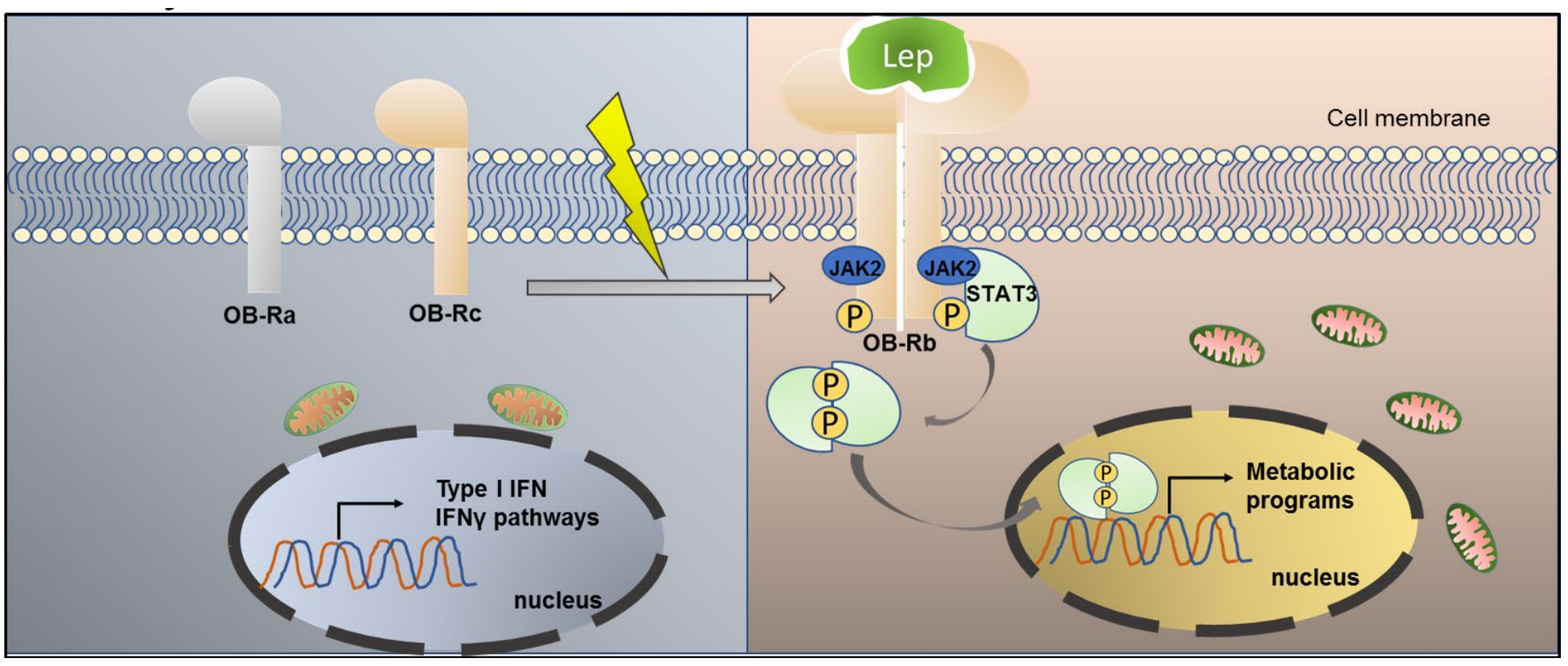


Figure 4.1 LEPR ${ }^{+}$HSCs under steady state condition becomes 'activated' in post-injury recovery (hypothetical).

A. Under homeostasis LEPR ${ }^{+} H S C$ s only express short isoforms of Lepr $(O B-R a \& O B-R c)$ and are characterized by a proinflammatory transcriptomic profile.

B. Hypothetically, LEPR ${ }^{+} H S C$ s may express long isoform of $\operatorname{Lepr}(O B-R b)$ as a result of stress-associated activation. 


\subsection{Future directions}

\subsubsection{Role(s) of LEP/LEPR in hematopoiesis under non-pathological conditions}

While our work suggested that LEPR can be used as a surface marker for functional long-term hematopoietic stem cells, it does not entirely rule out the possibility that LEPR could be a functional receptor under other different circumstances. Similarly, regarding the extensive findings on the multifunctional importance of LEPR-expressing BM niche cells for adult hematopoiesis, it would also be meaningful and clinically relevant to know whether the LEP ligand itself mediated these effects or if there were alternative cytokine cross-signaling pathways that acted through LEPR, for example like IL-6 signaling (Baumann et

al., 1996; Sadagurski et al., 2010). Furthermore, leptin has been reported to have a putative truncated site for DPP4 (O'Leary, Ou, \& Broxmeyer, 2013); hence, if LEP turns out to induce signaling on HSCs/HPCs and/or stromal cells it will be worthwhile to determine how the truncated version of leptin might alter leptin's functions on those cells.

Though we did not observe a bias in fate decision in young adult BM LEPR ${ }^{+} H S C s$, it is important to determine whether this will hold true as the organism ages. Our phenotyping data in middle-aged suggested that aging affected phenotypic LEPR ${ }^{+} H S C s$ and multiple lineage-biased subsets of LEPR ${ }^{+} M P P s$ to a greater extent than it does on LEPR- cells. Hence, it will be interesting to determine whether aging may also alter their functions differentially 
by performing in vitro CFU assay and in vivo quantitative competitive transplantation assays on a cell-to-cell basis.

On the other hand, based on our evidence that LEPR ${ }^{+} H S C$ s carry an embryoniclike transcriptomic profile and the fact that leptin plays a role in endothelial cell functions and angiogenesis (Park et al., 2001; Sierra-Honigmann et al., 1998), a critical question remaining is whether LEPR/LEPR signaling has any effects during the emergence of HSCs from the hemogenic endothelium. Would it be possible that the functional isoform Ob-Rb of Lepr got turned off once HSCs successfully emerged from embryonic endothelial cells? All of these warrant future in-depth studies as the answers to those questions might have potential implications both for the field of hematological malignancy research and HSC transplantation.

\subsubsection{Role(s) of LEP/LEPR in hematopoiesis under pathological/leukemic conditions}

For future work, it will be interesting and relevant to investigate further functional characteristics of stem cells with disrupted LEP/LEPR signaling. For instance, how would it affect the ability of the cells to differentiate versus maintaining a stem-like state? Would it affect one or multiple lineages and to which extent? Would the effect(s) be reversible? Would the effect(s) be similar under steady state versus pathological conditions? 
Given our findings in the present study, it could be logically inferred that LEPRexpressing leukemia blast cells might possess potent stemness, enriched for leukemia-initiating cells and hence more likely to be resistant to radiation and chemotherapeutic regimen. Since aging is associated with increased risks for malignancies particularly myeloid-biased leukemia, and LEPR-expressing HSCs/HPCs expanded phenotypically in older mice, it is clinically relevant to investigate more into their molecular mechanisms such as increased cell proliferation leading to functional exhaustion and higher risk for leukemic transformations. It will also be helpful to characterize their genetic and epigenetic programs under aged conditions, which can provide more insights into normal and abnormal stem cell aging.

In addition, provided that LEPR ${ }^{+} H S C$ s engrafted significantly better than LEPRcells upon irradiation and their highly proinflammatory transcriptome, it may be worthy to determine if LEPR ${ }^{+} H S C s$ are transcriptomically equipped under steadystate to respond to acute injury at a higher efficiency and faster rate. This could also be extrapolated into other situations such as in emergency hematopoiesis (e.g. acute infection) or even chronic infection in which the cells are constantly exposed to low-grade proinflammatory microenvironments. It is important to elucidate whether in such circumstances as leptin has any direct effects on HSCs and/or indirect effects through the BM niche, or perhaps a central vs. peripheral effect. A summary of what can be possibly done to further understand the role(s) 
of LEP/LEPR in hematopoiesis under normal and pathological conditions is outlined in table 4.2 . 


\begin{tabular}{|c|c|c|}
\hline \multicolumn{3}{|c|}{ Table 4.2. LEP/LEPR in hematopoiesis. What's next? } \\
\hline $\begin{array}{l}\text { Non-pathological } \\
\text { conditions }\end{array}$ & o & $\begin{array}{l}\text { Molecular mechanisms by which LEPR signaling } \\
\text { in stromal cells regulate expression of important } \\
\text { niche factors under steady state vs. post-injury } \\
\text { hematopoietic regeneration. } \\
\text { Is LEPR on HSCs just a surface marker or is } \\
\text { LEPR functional especially in context of post- } \\
\text { injury recovery? } \\
\text { Does the sources of LEP (e.g. systemic vs. local } \\
\text { BM adipocytes) matter? } \\
\text { Does LEP/LEPR play any roles in the } \\
\text { specification of HSCs during embryonic period } \\
\text { up until birth? And if so, by which mechanisms? } \\
\text { And how does maternal and fetal leptin } \\
\text { production affect these processes? } \\
\text { How may aging exert differential effects on } \\
\text { LEPR }{ }^{+} \text {stromal cells or LEPR+ HSCs vs. LEPR- } \\
\text { cells? } \\
\text { Does LEP signal through other cytokine } \\
\text { receptor(s) in the same family that may be } \\
\text { present on BM MSCs and/or HSCs? }\end{array}$ \\
\hline
\end{tabular}




\begin{tabular}{|c|c|c|}
\hline $\begin{array}{l}\text { Pathological } \\
\text { conditions/leukemia }\end{array}$ & 0 & $\begin{array}{l}\text { Do LEPR }{ }^{+} \text {HSCs/HPCs possess different } \\
\text { potentials for leukemic transformation from } \\
\text { LEPR- cells? How can other risk factors possibly } \\
\text { further modulate the difference? } \\
\text { There were discrepancies between MDS model } \\
\text { using Lep/- mice and patient studies. Was this } \\
\text { actually due to obesity alone as suggested or } \\
\text { the absence of LEP played additional roles? } \\
\text { While healthy mouse HSCs/MPPs were } \\
\text { reported to express predominantly only the short } \\
\text { isoforms under steady state, by which } \\
\text { mechanisms the leukemic blast cells for both } \\
\text { myeloid and lymphoid malignancies reactivated } \\
\text { LEPR long isoform? And how does this affect } \\
\text { their functional behaviors? Implications for } \\
\text { treatments? }\end{array}$ \\
\hline
\end{tabular}




\subsection{Conclusion}

LEP and LEPR signaling play important modulatory roles throughout an organism's life from conception to old age. LEPR resistance is well-recognized in obesity and aging (Myers et al., 2010; Sasaki, 2015). With a persistently increased prevalence of obesity and growth in the elderly populations worldwide, understanding how the different pathological conditions including hematologic malignancies and hematopoietic transplantation-associated complications biasedly affected these subsets of patients has become even more important. In this report, we noted and highlighted the work that provided proof-of-principle evidence for more future in-depth studies. A summary of what is known about LEP and LEPR in hematopoiesis can be found in Table 1.1. In brief, LEP/LEPR have been suggested to regulate stem cell numbers and functions particularly in the hematopoietic system under homeostasis, as well as post-injury regeneration and malignant transformation. Even though there are still some discrepancies between different studies or between animal and human data especially regarding LEPR and leukemic cells, the findings lay a strong foundation and create interesting questions to be answered in follow-up studies. Future work that elucidates and provides insight into mechanisms of LEP/LEPR interactions is warranted and may provide potential implications for therapeutic approaches to enhance healthcare. 


\section{Graphical Abstract}

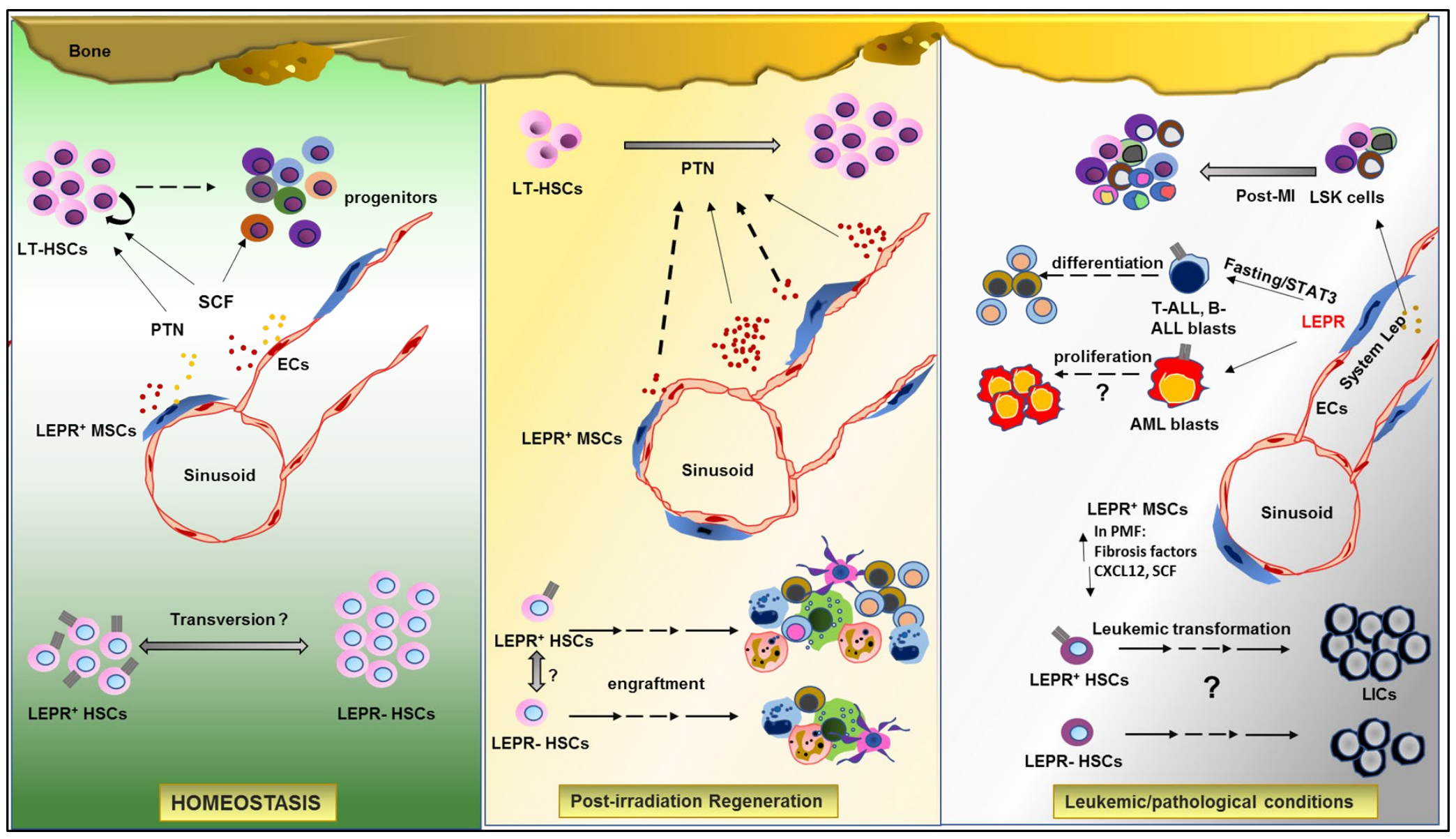




\section{Graphical Abstract}

- Under steady state, only a minor subset of long-term hematopoietic stem cells (HSCs) express LEPR

- Upon irradiation, LEPR ${ }^{+} H S C$ s exhibited robust repopulating capacity in long-term engraftment studies that outcompeted LEPR-HSCs

- $\mathrm{LEPR}^{+}$stromal cells secrete critical niche factors including stem cell factor (SCF) and pleiotrophin (PTN) to support HSCs and progenitor cells

- LEPR signaling mediated protective effects of fasting in ALL but not AML leukemias 


\section{REFERENCES}

Abarrategi, A., Mian, S. A., Passaro, D., Rouault-Pierre, K., Grey, W., \& Bonnet, D. (2018). Modeling the human bone marrow niche in mice: From host bone marrow engraftment to bioengineering approaches. J Exp Med, 215(3), 729-743. doi:10.1084/jem.20172139

Adler, B. J., Green, D. E., Pagnotti, G. M., Chan, M. E., \& Rubin, C. T. (2014). High fat diet rapidly suppresses B lymphopoiesis by disrupting the supportive capacity of the bone marrow niche. PLoS One, 9(3), e90639. doi:10.1371/journal.pone.0090639

Adolfsson, J., Mansson, R., Buza-Vidas, N., Hultquist, A., Liuba, K., Jensen, C. T., . . Jacobsen, S. E. (2005). Identification of Flt3+ lympho-myeloid stem cells lacking erythro-megakaryocytic potential a revised road map for adult blood lineage commitment. Cell, 121(2), 295-306.

doi:10.1016/j.cell.2005.02.013

Alpdogan, O., Eng, J. M., Muriglan, S. J., Willis, L. M., Hubbard, V. M., Tjoe, K. H., . . van den Brink, M. R. (2005). Interleukin-15 enhances immune reconstitution after allogeneic bone marrow transplantation. Blood, 105(2), 865-873. doi:10.1182/blood-2003-09-3344

Ambrosi, T. H., Scialdone, A., Graja, A., Gohlke, S., Jank, A. M., Bocian, C., . . . Schulz, T. J. (2017). Adipocyte Accumulation in the Bone Marrow during Obesity and Aging Impairs Stem Cell-Based Hematopoietic and Bone Regeneration. Cell Stem Cell, 20(6), 771-784 e776.

doi:10.1016/j.stem.2017.02.009 
Anasetti, C., Logan, B. R., Lee, S. J., Waller, E. K., Weisdorf, D. J., Wingard, J. R., . . Marrow Transplant Clinical Trials, N. (2012). Peripheral-blood stem cells versus bone marrow from unrelated donors. N Engl J Med, 367(16), 1487-1496. doi:10.1056/NEJMoa1203517

Antonchuk, J., Sauvageau, G., \& Humphries, R. K. (2002). HOXB4-induced expansion of adult hematopoietic stem cells ex vivo. Cell, 109(1), 39-45. doi:10.1016/s0092-8674(02)00697-9

Asada, N., Kunisaki, Y., Pierce, H., Wang, Z., Fernandez, N. F., Birbrair, A., . . . Frenette, P. S. (2017). Differential cytokine contributions of perivascular haematopoietic stem cell niches. Nat Cell Biol, 19(3), 214-223. doi:10.1038/ncb3475

Bahary, N., Leibel, R. L., Joseph, L., \& Friedman, J. M. (1990). Molecular mapping of the mouse db mutation. Proc Natl Acad Sci U S A, 87(21), 8642-8646. doi:10.1073/pnas.87.21.8642

Ballen, K. K., Gluckman, E., \& Broxmeyer, H. E. (2013). Umbilical cord blood transplantation: the first 25 years and beyond. Blood, 122(4), 491-498. doi:10.1182/blood-2013-02-453175

Barb, C. R., Hausman, G. J., \& Lents, C. A. (2008). Energy metabolism and leptin: effects on neuroendocrine regulation of reproduction in the gilt and sow. Reprod Domest Anim, 43 Suppl 2, 324-330. doi:10.1111/j.14390531.2008.01173.x 
Barnes, D. W., Corp, M. J., Loutit, J. F., \& Neal, F. E. (1956). Treatment of murine leukaemia with $\mathrm{X}$ rays and homologous bone marrow; preliminary communication. Br Med J, 2(4993), 626-627. doi:10.1136/bmj.2.4993.626

Baumann, H., Morella, K. K., White, D. W., Dembski, M., Bailon, P. S., Kim, H., . . . Tartaglia, L. A. (1996). The full-length leptin receptor has signaling capabilities of interleukin 6-type cytokine receptors. Proc Natl Acad Sci U S A, 93(16), 8374-8378. doi:10.1073/pnas.93.16.8374

Becker, A. J., Mc, C. E., \& Till, J. E. (1963). Cytological demonstration of the clonal nature of spleen colonies derived from transplanted mouse marrow cells. Nature, 197, 452-454. doi:10.1038/197452a0

Bejar, R., Stevenson, K., Abdel-Wahab, O., Galili, N., Nilsson, B., GarciaManero, G., . . Ebert, B. L. (2011). Clinical effect of point mutations in myelodysplastic syndromes. N Engl J Med, 364(26), 2496-2506. doi:10.1056/NEJMoa1013343

Bennett, B. D., Solar, G. P., Yuan, J. Q., Mathias, J., Thomas, G. R., \& Matthews, W. (1996). A role for leptin and its cognate receptor in hematopoiesis. Curr Biol, 6(9), 1170-1180. doi:10.1016/s09609822(02)70684-2

Bensinger, W. I., Martin, P. J., Storer, B., Clift, R., Forman, S. J., Negrin, R., . . . Appelbaum, F. R. (2001). Transplantation of bone marrow as compared with peripheral-blood cells from HLA-identical relatives in patients with hematologic cancers. N Engl J Med, 344(3), 175-181.

doi:10.1056/NEJM200101183440303 
Bertoncello, I., Hodgson, G. S., \& Bradley, T. R. (1985). Multiparameter analysis of transplantable hemopoietic stem cells: I. The separation and enrichment of stem cells homing to marrow and spleen on the basis of rhodamine-123 fluorescence. Exp Hematol, 13(10), 999-1006. Retrieved from https://www.ncbi.nlm.nih.gov/pubmed/2865163

Bertrand, J. Y., Cisson, J. L., Stachura, D. L., \& Traver, D. (2010). Notch signaling distinguishes 2 waves of definitive hematopoiesis in the zebrafish embryo. Blood, 115(14), 2777-2783. doi:10.1182/blood-2009-09244590

Blaise, D., Kuentz, M., Fortanier, C., Bourhis, J. H., Milpied, N., Sutton, L., . . . Michallet, M. (2000). Randomized trial of bone marrow versus lenograstim-primed blood cell allogeneic transplantation in patients with early-stage leukemia: a report from the Societe Francaise de Greffe de Moelle. J Clin Oncol, 18(3), 537-546. doi:10.1200/JCO.2000.18.3.537

Bock, T. A., Orlic, D., Dunbar, C. E., Broxmeyer, H. E., \& Bodine, D. M. (1995). Improved engraftment of human hematopoietic cells in severe combined immunodeficient (SCID) mice carrying human cytokine transgenes. J Exp Med, 182(6), 2037-2043. doi:10.1084/jem.182.6.2037

Boitano, A. E., Wang, J., Romeo, R., Bouchez, L. C., Parker, A. E., Sutton, S. E., ... Cooke, M. P. (2010). Aryl hydrocarbon receptor antagonists promote the expansion of human hematopoietic stem cells. Science, 329(5997), 1345-1348. doi:10.1126/science.1191536 
Bowie, M. B., Kent, D. G., Dykstra, B., McKnight, K. D., McCaffrey, L., Hoodless, P. A., \& Eaves, C. J. (2007). Identification of a new intrinsically timed developmental checkpoint that reprograms key hematopoietic stem cell properties. Proc Natl Acad Sci U S A, 104(14), 5878-5882. doi:10.1073/pnas.0700460104

Broxmeyer, H. E. (2008). Cord blood hematopoietic stem cell transplantation. In StemBook. Cambridge (MA).

Broxmeyer, H. E., Hoggatt, J., O'Leary, H. A., Mantel, C., Chitteti, B. R., Cooper, S., . . Campbell, T. B. (2012). Dipeptidylpeptidase 4 negatively regulates colony-stimulating factor activity and stress hematopoiesis. Nat Med, 18(12), 1786-1796. doi:10.1038/nm.2991

Broxmeyer, H. E., Li, J., Hangoc, G., Cooper, S., Tao, W., Mantel, C., . . de Sauvage, F. J. (2007). Regulation of myeloid progenitor cell proliferation/survival by IL-31 receptor and IL-31. Exp Hematol, 35(4 Suppl 1), 78-86. doi:10.1016/j.exphem.2007.01.028

Broxmeyer, H. E., Liu, Y., Kapur, R., Orschell, C. M., Aljoufi, A., Ropa, J. P., . . Capitano, M. L. (2020). Fate of Hematopoiesis During Aging. What Do We Really Know, and What are its Implications? Stem Cell Rev Rep, 16(6), 1020-1048. doi:10.1007/s12015-020-10065-y

Bruserud, O., Huang, T. S., Glenjen, N., Gjertsen, B. T., \& Foss, B. (2002). Leptin in human acute myelogenous leukemia: studies of in vivo levels and in vitro effects on native functional leukemia blasts. Haematologica, 
87(6), 584-595. Retrieved from

https://www.ncbi.nlm.nih.gov/pubmed/12031914

Busque, L., Buscarlet, M., Mollica, L., \& Levine, R. L. (2018). Concise Review:

Age-Related Clonal Hematopoiesis: Stem Cells Tempting the Devil. Stem Cells, 36(9), 1287-1294. doi:10.1002/stem.2845

Busque, L., Patel, J. P., Figueroa, M. E., Vasanthakumar, A., Provost, S., Hamilou, Z., . . Levine, R. L. (2012). Recurrent somatic TET2 mutations in normal elderly individuals with clonal hematopoiesis. Nat Genet, 44(11), 1179-1181. doi:10.1038/ng.2413

Calvi, L. M., Adams, G. B., Weibrecht, K. W., Weber, J. M., Olson, D. P., Knight, M. C., . . S Scadden, D. T. (2003). Osteoblastic cells regulate the haematopoietic stem cell niche. Nature, 425(6960), 841-846. doi:10.1038/nature02040

Campfield, L. A., Smith, F. J., Guisez, Y., Devos, R., \& Burn, P. (1995). Recombinant mouse OB protein: evidence for a peripheral signal linking adiposity and central neural networks. Science, 269(5223), 546-549. doi:10.1126/science. 7624778

Cancer Genome Atlas Research, N., Ley, T. J., Miller, C., Ding, L., Raphael, B. J., Mungall, A. J., . . Eley, G. (2013). Genomic and epigenomic landscapes of adult de novo acute myeloid leukemia. N Engl J Med, 368(22), 2059-2074. doi:10.1056/NEJMoa1301689

Capitano, M. L., Hangoc, G., Cooper, S., \& Broxmeyer, H. E. (2015). Mild Heat Treatment Primes Human CD34(+) Cord Blood Cells for Migration Toward 
SDF-1alpha and Enhances Engraftment in an NSG Mouse Model. Stem Cells, 33(6), 1975-1984. doi:10.1002/stem.1988

Capitano, M. L., Mohamad, S. F., Cooper, S., Guo, B., Huang, X., Gunawan, A.M., Sampson, C., Ropa, J., Srour, E.F., Orschell, C.M., \& Broxmeyer, H.E. (2020). Mitigating oxygen stress enhances aged mouse hematopoietic stem cell numbers and function. Journal of Clinical Investigation, In Press.

Capitano, M. L., Mor-Vaknin, N., Saha, A. K., Cooper, S., Legendre, M., Guo, H., ... Broxmeyer, H. E. (2019). Secreted nuclear protein DEK regulates hematopoiesis through CXCR2 signaling. J Clin Invest, 129(6), 25552570. doi:10.1172/JCl127460

Challen, G. A., Sun, D., Jeong, M., Luo, M., Jelinek, J., Berg, J. S., . . Goodell, M. A. (2011). Dnmt3a is essential for hematopoietic stem cell differentiation. Nat Genet, 44(1), 23-31. doi:10.1038/ng.1009

Chan, C. K., Chen, C. C., Luppen, C. A., Kim, J. B., DeBoer, A. T., Wei, K., . . Weissman, I. L. (2009). Endochondral ossification is required for haematopoietic stem-cell niche formation. Nature, 457(7228), 490-494. doi:10.1038/nature07547

Chaurasia, P., Gajzer, D. C., Schaniel, C., D'Souza, S., \& Hoffman, R. (2014). Epigenetic reprogramming induces the expansion of cord blood stem cells. J Clin Invest, 124(6), 2378-2395. doi:10.1172/JCI70313

Chen, Y., Yao, C., Teng, Y., Jiang, R., Huang, X., Liu, S., . . Guo, B. (2019). Phorbol ester induced ex vivo expansion of rigorously-defined phenotypic 
but not functional human cord blood hematopoietic stem cells: a cautionary tale demonstrating that phenotype does not always recapitulate stem cell function. Leukemia, 33(12), 2962-2966. doi:10.1038/s41375019-0528-3

Cheung, C. C., Clifton, D. K., \& Steiner, R. A. (1997). Proopiomelanocortin neurons are direct targets for leptin in the hypothalamus. Endocrinology, 138(10), 4489-4492. doi:10.1210/endo.138.10.5570

Chou, S. H., \& Mantzoros, C. (2014). 20 years of leptin: role of leptin in human reproductive disorders. J Endocrinol, 223(1), T49-62. doi:10.1530/JOE-140245

Christensen, J. L., \& Weissman, I. L. (2001). Flk-2 is a marker in hematopoietic stem cell differentiation: a simple method to isolate long-term stem cells. Proc Natl Acad Sci U S A, 98(25), 14541-14546.

doi:10.1073/pnas.261562798

Christopherson, K. W., 2nd, Hangoc, G., \& Broxmeyer, H. E. (2002). Cell surface peptidase CD26/dipeptidylpeptidase IV regulates CXCL12/stromal cellderived factor-1 alpha-mediated chemotaxis of human cord blood CD34+ progenitor cells. J Immunol, 169(12), 7000-7008.

doi:10.4049/jimmunol.169.12.7000

Christopherson, K. W., 2nd, Hangoc, G., Mantel, C. R., \& Broxmeyer, H. E. (2004). Modulation of hematopoietic stem cell homing and engraftment by CD26. Science, 305(5686), 1000-1003. doi:10.1126/science.1097071 
Cioffi, J. A., Shafer, A. W., Zupancic, T. J., Smith-Gbur, J., Mikhail, A., Platika, D., \& Snodgrass, H. R. (1996). Novel B219/OB receptor isoforms: possible role of leptin in hematopoiesis and reproduction. Nat Med, 2(5), 585-589. doi:10.1038/nm0596-585

Claycombe, K., King, L. E., \& Fraker, P. J. (2008). A role for leptin in sustaining lymphopoiesis and myelopoiesis. Proc Natl Acad Sci U S A, 105(6), 20172021. doi:10.1073/pnas.0712053105

Cohen, S., Roy, J., Lachance, S., Delisle, J. S., Marinier, A., Busque, L., . . . Sauvageau, G. (2020). Hematopoietic stem cell transplantation using single UM171-expanded cord blood: a single-arm, phase 1-2 safety and feasibility study. Lancet Haematol, 7(2), e134-e145. doi:10.1016/S23523026(19)30202-9

Comazzetto, S., Murphy, M. M., Berto, S., Jeffery, E., Zhao, Z., \& Morrison, S. J. (2019). Restricted Hematopoietic Progenitors and Erythropoiesis Require SCF from Leptin Receptor+ Niche Cells in the Bone Marrow. Cell Stem Cell, 24(3), 477-486 e476. doi:10.1016/j.stem.2018.11.022

Copelan, E. A. (2006). Hematopoietic stem-cell transplantation. N Engl J Med, 354(17), 1813-1826. doi:10.1056/NEJMra052638

Corces-Zimmerman, M. R., Hong, W. J., Weissman, I. L., Medeiros, B. C., \& Majeti, R. (2014). Preleukemic mutations in human acute myeloid leukemia affect epigenetic regulators and persist in remission. Proc Natl Acad Sci U S A, 111(7), 2548-2553. doi:10.1073/pnas.1324297111 
Couronne, L., Bastard, C., \& Bernard, O. A. (2012). TET2 and DNMT3A mutations in human T-cell lymphoma. N Engl J Med, 366(1), 95-96. doi:10.1056/NEJMc1111708

Crean-Tate, K. K., \& Reizes, O. (2018). Leptin Regulation of Cancer Stem Cells in Breast and Gynecologic Cancer. Endocrinology, 159(8), 3069-3080. doi:10.1210/en.2018-00379

Cumano, A., Ferraz, J. C., Klaine, M., Di Santo, J. P., \& Godin, I. (2001). Intraembryonic, but not yolk sac hematopoietic precursors, isolated before circulation, provide long-term multilineage reconstitution. Immunity, 15(3), 477-485. doi:10.1016/s1074-7613(01)00190-x

Cunningham-Rundles, S., McNeeley, D. F., \& Moon, A. (2005). Mechanisms of nutrient modulation of the immune response. J Allergy Clin Immunol, 115(6), 1119-1128; quiz 1129. doi:10.1016/j.jaci.2005.04.036

Dar, A., Schajnovitz, A., Lapid, K., Kalinkovich, A., Itkin, T., Ludin, A., . . . Lapidot, T. (2011). Rapid mobilization of hematopoietic progenitors by AMD3100 and catecholamines is mediated by CXCR4-dependent SDF-1 release from bone marrow stromal cells. Leukemia, 25(8), 1286-1296. doi:10.1038/leu.2011.62

Dausset, J. (1958). [Iso-leuko-antibodies]. Acta Haematol, 20(1-4), 156-166. doi:10.1159/000205478

de Koning, C., Nierkens, S., \& Boelens, J. J. (2016). Strategies before, during, and after hematopoietic cell transplantation to improve T-cell immune 
reconstitution. Blood, 128(23), 2607-2615. doi:10.1182/blood-2016-06724005

De Rosa, V., Procaccini, C., Cali, G., Pirozzi, G., Fontana, S., Zappacosta, S., . . . Matarese, G. (2007). A key role of leptin in the control of regulatory T cell proliferation. Immunity, 26(2), 241-255. doi:10.1016/j.immuni.2007.01.011 Decker, M., Martinez-Morentin, L., Wang, G., Lee, Y., Liu, Q., Leslie, J., \& Ding, L. (2017). Leptin-receptor-expressing bone marrow stromal cells are myofibroblasts in primary myelofibrosis. Nat Cell Biol, 19(6), 677-688. doi:10.1038/ncb3530

Dieterlen-Lievre, F. (1975). On the origin of haemopoietic stem cells in the avian embryo: an experimental approach. J Embryol Exp Morphol, 33(3), 607619. Retrieved from https://www.ncbi.nlm.nih.gov/pubmed/1176862

Ding, L., Saunders, T. L., Enikolopov, G., \& Morrison, S. J. (2012). Endothelial and perivascular cells maintain haematopoietic stem cells. Nature, 481(7382), 457-462. doi:10.1038/nature10783

Doulatov, S., Notta, F., Laurenti, E., \& Dick, J. E. (2012). Hematopoiesis: a human perspective. Cell Stem Cell, 10(2), 120-136. doi:10.1016/j.stem.2012.01.006

Duncan, B. K., \& Miller, J. H. (1980). Mutagenic deamination of cytosine residues in DNA. Nature, 287(5782), 560-561. doi:10.1038/287560a0

Dykstra, B., Olthof, S., Schreuder, J., Ritsema, M., \& de Haan, G. (2011). Clonal analysis reveals multiple functional defects of aged murine hematopoietic stem cells. J Exp Med, 208(13), 2691-2703. doi:10.1084/jem.20111490 
Dzierzak, E., \& Medvinsky, A. (1995). Mouse embryonic hematopoiesis. Trends Genet, 11(9), 359-366. doi:10.1016/s0168-9525(00)89107-6

Ergen, A. V., Boles, N. C., \& Goodell, M. A. (2012). Rantes/Ccl5 influences hematopoietic stem cell subtypes and causes myeloid skewing. Blood, 119(11), 2500-2509. doi:10.1182/blood-2011-11-391730

Espin-Palazon, R., Stachura, D. L., Campbell, C. A., Garcia-Moreno, D., Del Cid, N., Kim, A. D., . . Traver, D. (2014). Proinflammatory signaling regulates hematopoietic stem cell emergence. Cell, 159(5), 1070-1085. doi:10.1016/j.cell.2014.10.031

Espin-Palazon, R., \& Traver, D. (2016). The NF-kappaB family: Key players during embryonic development and HSC emergence. Exp Hematol, 44(7), 519-527. doi:10.1016/j.exphem.2016.03.010

Fares, I., Chagraoui, J., Gareau, Y., Gingras, S., Ruel, R., Mayotte, N., . . Sauvageau, G. (2014). Cord blood expansion. Pyrimidoindole derivatives are agonists of human hematopoietic stem cell self-renewal. Science, 345(6203), 1509-1512. doi:10.1126/science.1256337

Filippi, B. M., \& Lam, T. K. (2014). Leptin and aging. Aging (Albany NY), 6(2), 8283. doi:10.18632/aging.100637

Forsberg, E. C., Prohaska, S. S., Katzman, S., Heffner, G. C., Stuart, J. M., \& Weissman, I. L. (2005). Differential expression of novel potential regulators in hematopoietic stem cells. PLoS Genet, 1(3), e28. doi:10.1371/journal.pgen.0010028 
Frasca, D., \& Blomberg, B. B. (2017). Adipose Tissue Inflammation Induces B Cell Inflammation and Decreases B Cell Function in Aging. Front Immunol, 8, 1003. doi:10.3389/fimmu.2017.01003

Frasca, D., Diaz, A., Romero, M., \& Blomberg, B. B. (2020). Leptin induces immunosenescence in human B cells. Cell Immunol, 348, 103994. doi:10.1016/j.cellimm.2019.103994

Friedman, J. (2016). The long road to leptin. J Clin Invest, 126(12), 4727-4734. doi:10.1172/JCI91578

Friedman, J. M. (2019). Leptin and the endocrine control of energy balance. Nat Metab, 1(8), 754-764. doi:10.1038/s42255-019-0095-y

Friedman, J. M., \& Halaas, J. L. (1998). Leptin and the regulation of body weight in mammals. Nature, 395(6704), 763-770. doi:10.1038/27376

Friedman, J. M., Leibel, R. L., Siegel, D. S., Walsh, J., \& Bahary, N. (1991). Molecular mapping of the mouse ob mutation. Genomics, 11(4), 10541062. doi:10.1016/0888-7543(91)90032-a

Frodermann, V., Rohde, D., Courties, G., Severe, N., Schloss, M. J., Amatullah, H., . . Nahrendorf, M. (2019). Exercise reduces inflammatory cell production and cardiovascular inflammation via instruction of hematopoietic progenitor cells. Nat Med, 25(11), 1761-1771. doi:10.1038/s41591-019-0633-x

Gabriely, I., Ma, X. H., Yang, X. M., Rossetti, L., \& Barzilai, N. (2002). Leptin resistance during aging is independent of fat mass. Diabetes, 51(4), 10161021. doi:10.2337/diabetes.51.4.1016 
Gainsford, T., Willson, T. A., Metcalf, D., Handman, E., McFarlane, C., Ng, A., . . . Hilton, D. J. (1996). Leptin can induce proliferation, differentiation, and functional activation of hemopoietic cells. Proc Natl Acad Sci U S A, 93(25), 14564-14568. doi:10.1073/pnas.93.25.14564

Galvin, A., Weglarz, M., Folz-Donahue, K., Handley, M., Baum, M., Mazzola, M., ... Silberstein, L. (2019). Cell Cycle Analysis of Hematopoietic Stem and Progenitor Cells by Multicolor Flow Cytometry. Curr Protoc Cytom, 87(1), e50. doi:10.1002/cpcy.50

Garcia-Porrero, J. A., Godin, I. E., \& Dieterlen-Lievre, F. (1995). Potential intraembryonic hemogenic sites at pre-liver stages in the mouse. Anat Embryol (Berl), 192(5), 425-435. doi:10.1007/BF00240375

Gekas, C., Dieterlen-Lievre, F., Orkin, S. H., \& Mikkola, H. K. (2005). The placenta is a niche for hematopoietic stem cells. Dev Cell, 8(3), 365-375. doi:10.1016/j.devcel.2004.12.016

Godin, I., Dieterlen-Lievre, F., \& Cumano, A. (1995). Emergence of multipotent hemopoietic cells in the yolk sac and paraaortic splanchnopleura in mouse embryos, beginning at 8.5 days postcoitus. Proc Natl Acad Sci U S A, 92(3), 773-777. doi:10.1073/pnas.92.3.773

Gong, J. K. (1978). Endosteal marrow: a rich source of hematopoietic stem cells. Science, 199(4336), 1443-1445. doi:10.1126/science.75570

Goodell, M. A., Brose, K., Paradis, G., Conner, A. S., \& Mulligan, R. C. (1996). Isolation and functional properties of murine hematopoietic stem cells that 
are replicating in vivo. $J$ Exp Med, 183(4), 1797-1806.

doi:10.1084/jem.183.4.1797

Gorska, E., Popko, K., Stelmaszczyk-Emmel, A., Ciepiela, O., Kucharska, A., \& Wasik, M. (2010). Leptin receptors. Eur J Med Res, 15 Supp/ 2, 50-54. doi:10.1186/2047-783x-15-s2-50

Gorska, E., Popko, K., \& Wasik, M. (2013). Leptin receptor in childhood acute leukemias. Adv Exp Med Biol, 756, 155-161. doi:10.1007/978-94-0074549-0_20

Grass, J. A., Boyer, M. E., Pal, S., Wu, J., Weiss, M. J., \& Bresnick, E. H. (2003). GATA-1-dependent transcriptional repression of GATA-2 via disruption of positive autoregulation and domain-wide chromatin remodeling. Proc Natl Acad Sci U S A, 100(15), 8811-8816. doi:10.1073/pnas.1432147100

Guidi, N., Sacma, M., Standker, L., Soller, K., Marka, G., Eiwen, K., . . Geiger, H. (2017). Osteopontin attenuates aging-associated phenotypes of hematopoietic stem cells. EMBO J, 36(10), 1463.

doi:10.15252/embj.201796968

Guo, B., Huang, X., Cooper, S., \& Broxmeyer, H. E. (2017). Glucocorticoid hormone-induced chromatin remodeling enhances human hematopoietic stem cell homing and engraftment. Nat Med, 23(4), 424-428. doi: $10.1038 / \mathrm{nm} .4298$

Guo, B., Huang, X., Lee, M. R., Lee, S. A., \& Broxmeyer, H. E. (2018). Antagonism of PPAR-gamma signaling expands human hematopoietic 
stem and progenitor cells by enhancing glycolysis. Nat Med, 24(3), 360367. doi: $10.1038 / \mathrm{nm} .4477$

Gupta, M. K., Vethe, H., Softic, S., Rao, T. N., Wagh, V., Shirakawa, J., . . . Kulkarni, R. N. (2020). Leptin Receptor Signaling Regulates Protein Synthesis Pathways and Neuronal Differentiation in Pluripotent Stem Cells. Stem Cell Reports, 15(5), 1067-1079. doi:10.1016/j.stemcr.2020.10.001

Gupta, S., Agrawal, S., \& Gollapudi, S. (2013). Increased activation and cytokine secretion in B cells stimulated with leptin in aged humans. Immun Ageing, 10(1), 3. doi:10.1186/1742-4933-10-3

Hamey, F. K., Nestorowa, S., Kinston, S. J., Kent, D. G., Wilson, N. K., \& Gottgens, B. (2017). Reconstructing blood stem cell regulatory network models from single-cell molecular profiles. Proc Natl Acad Sci U S A, 114(23), 5822-5829. doi:10.1073/pnas.1610609114

Hayes, S. C., Rowbottom, D., Davies, P. S., Parker, T. W., \& Bashford, J. (2003). Immunological changes after cancer treatment and participation in an exercise program. Med Sci Sports Exerc, 35(1), 2-9.

doi:10.1097/00005768-200301000-00002

Himburg, H. A., Termini, C. M., Schlussel, L., Kan, J., Li, M., Zhao, L., . . Chute, J. P. (2018). Distinct Bone Marrow Sources of Pleiotrophin Control Hematopoietic Stem Cell Maintenance and Regeneration. Cell Stem Cell, 23(3), 370-381 e375. doi:10.1016/j.stem.2018.07.003 
Hoang, M. L., Kinde, I., Tomasetti, C., McMahon, K. W., Rosenquist, T. A., Grollman, A. P., . . Papadopoulos, N. (2016). Genome-wide quantification of rare somatic mutations in normal human tissues using massively parallel sequencing. Proc Natl Acad Sci U S A, 113(35), 98469851. doi:10.1073/pnas.1607794113

Huang, X., Guo, B., Capitano, M., \& Broxmeyer, H. E. (2019). Past, present, and future efforts to enhance the efficacy of cord blood hematopoietic cell transplantation. F1000Res, 8. doi:10.12688/f1000research.20002.1

Huang, X., Guo, B., Liu, S., Wan, J., \& Broxmeyer, H. E. (2018). Neutralizing negative epigenetic regulation by HDAC5 enhances human haematopoietic stem cell homing and engraftment. Nat Commun, 9(1), 2741. doi:10.1038/s41467-018-05178-5

Huang, X., Lee, M. R., Cooper, S., Hangoc, G., Hong, K. S., Chung, H. M., \& Broxmeyer, H. E. (2016). Activation of OCT4 enhances ex vivo expansion of human cord blood hematopoietic stem and progenitor cells by regulating HOXB4 expression. Leukemia, 30(1), 144-153. doi:10.1038/leu.2015.189 Inamoto, Y., Fefer, A., Sandmaier, B. M., Gooley, T. A., Warren, E. H., Petersdorf, S. H., . . Flowers, M. E. (2011). A phase I/II study of chemotherapy followed by donor lymphocyte infusion plus interleukin-2 for relapsed acute leukemia after allogeneic hematopoietic cell transplantation. Biol Blood Marrow Transplant, 17(9), 1308-1315. doi:10.1016/j.bbmt.2011.01.004 
Inui, A. (1999). Feeding and body-weight regulation by hypothalamic neuropeptides--mediation of the actions of leptin. Trends Neurosci, 22(2), 62-67. doi:10.1016/s0166-2236(98)01292-2

Jaiswal, S., \& Ebert, B. L. (2019). Clonal hematopoiesis in human aging and disease. Science, 366(6465). doi:10.1126/science.aan4673

Jaiswal, S., Fontanillas, P., Flannick, J., Manning, A., Grauman, P. V., Mar, B. G., . . Ebert, B. L. (2014). Age-related clonal hematopoiesis associated with adverse outcomes. N Engl J Med, 371(26), 2488-2498. doi:10.1056/NEJMoa1408617

Jan, M., Snyder, T. M., Corces-Zimmerman, M. R., Vyas, P., Weissman, I. L., Quake, S. R., \& Majeti, R. (2012). Clonal evolution of preleukemic hematopoietic stem cells precedes human acute myeloid leukemia. Sci Trans/ Med, 4(149), 149ra118. doi:10.1126/scitransImed.3004315 Katayama, Y., Battista, M., Kao, W. M., Hidalgo, A., Peired, A. J., Thomas, S. A., \& Frenette, P. S. (2006). Signals from the sympathetic nervous system regulate hematopoietic stem cell egress from bone marrow. Cell, 124(2), 407-421. doi:10.1016/j.cell.2005.10.041

Kennedy-Nasser, A. A., Ku, S., Castillo-Caro, P., Hazrat, Y., Wu, M. F., Liu, H., . . Bollard, C. M. (2014). Ultra low-dose IL-2 for GVHD prophylaxis after allogeneic hematopoietic stem cell transplantation mediates expansion of regulatory $T$ cells without diminishing antiviral and antileukemic activity. Clin Cancer Res, 20(8), 2215-2225. doi:10.1158/1078-0432.CCR-13-3205 
Khaddour, K., Hana, C. K., \& Mewawalla, P. (2020). Hematopoietic Stem Cell Transplantation. In StatPearls. Treasure Island (FL).

Kiel, M. J., Yilmaz, O. H., Iwashita, T., Yilmaz, O. H., Terhorst, C., \& Morrison, S. J. (2005). SLAM family receptors distinguish hematopoietic stem and progenitor cells and reveal endothelial niches for stem cells. Cell, 121(7), 1109-1121. doi:10.1016/j.cell.2005.05.026

Kim, C. H., Qu, C. K., Hangoc, G., Cooper, S., Anzai, N., Feng, G. S., \& Broxmeyer, H. E. (1999). Abnormal chemokine-induced responses of immature and mature hematopoietic cells from motheaten mice implicate the protein tyrosine phosphatase SHP-1 in chemokine responses. J Exp Med, 190(5), 681-690. doi:10.1084/jem.190.5.681

Kim, I., He, S., Yilmaz, O. H., Kiel, M. J., \& Morrison, S. J. (2006). Enhanced purification of fetal liver hematopoietic stem cells using SLAM family receptors. Blood, 108(2), 737-744. doi:10.1182/blood-2005-10-4135

Ko, M., Bandukwala, H. S., An, J., Lamperti, E. D., Thompson, E. C., Hastie, R., . . Rao, A. (2011). Ten-Eleven-Translocation 2 (TET2) negatively regulates homeostasis and differentiation of hematopoietic stem cells in mice. Proc Natl Acad Sci U S A, 108(35), 14566-14571.

doi:10.1073/pnas.1112317108

Koenig, S., Luheshi, G. N., Wenz, T., Gerstberger, R., Roth, J., \& Rummel, C. (2014). Leptin is involved in age-dependent changes in response to systemic inflammation in the rat. Brain Behav Immun, 36, 128-138. doi:10.1016/j.bbi.2013.10.019 
Konopleva, M., Mikhail, A., Estrov, Z., Zhao, S., Harris, D., Sanchez-Williams, G., ... Andreeff, M. (1999). Expression and function of leptin receptor isoforms in myeloid leukemia and myelodysplastic syndromes: proliferative and anti-apoptotic activities. Blood, 93(5), 1668-1676. Retrieved from https://www.ncbi.nlm.nih.gov/pubmed/10029596 Kraakman, M. J., Kammoun, H. L., Dragoljevic, D., Al-Sharea, A., Lee, M. K. S., Flynn, M. C., . . Murphy, A. J. (2018). Leptin-deficient obesity prolongs survival in a murine model of myelodysplastic syndrome. Haematologica, 103(4), 597-606. doi:10.3324/haematol.2017.181958

Krings, A., Rahman, S., Huang, S., Lu, Y., Czernik, P. J., \& Lecka-Czernik, B. (2012). Bone marrow fat has brown adipose tissue characteristics, which are attenuated with aging and diabetes. Bone, 50(2), 546-552. doi:10.1016/j.bone.2011.06.016

Krosl, J., Austin, P., Beslu, N., Kroon, E., Humphries, R. K., \& Sauvageau, G. (2003). In vitro expansion of hematopoietic stem cells by recombinant TAT-HOXB4 protein. Nat Med, 9(11), 1428-1432. doi:10.1038/nm951

Kumano, K., Chiba, S., Kunisato, A., Sata, M., Saito, T., Nakagami-Yamaguchi, E., . . Hirai, H. (2003). Notch1 but not Notch2 is essential for generating hematopoietic stem cells from endothelial cells. Immunity, 18(5), 699-711. doi:10.1016/s1074-7613(03)00117-1

Kumaravelu, P., Hook, L., Morrison, A. M., Ure, J., Zhao, S., Zuyev, S., . . . Medvinsky, A. (2002). Quantitative developmental anatomy of definitive haematopoietic stem cells/long-term repopulating units (HSC/RUs): role of 
the aorta-gonad-mesonephros (AGM) region and the yolk sac in colonisation of the mouse embryonic liver. Development, 129(21), 48914899. Retrieved from https://www.ncbi.nlm.nih.gov/pubmed/12397098

Kurtovic, S., Ng, T. T., Gupta, A., Arumugaswami, V., Chaiboonma, K. L., Aminzadeh, M. A., ... Talavera-Adame, D. (2015). Leptin enhances endothelial cell differentiation and angiogenesis in murine embryonic stem cells. Microvasc Res, 97, 65-74. doi:10.1016/j.mvr.2014.09.004

La Cava, A., \& Matarese, G. (2004). The weight of leptin in immunity. Nat Rev Immunol, 4(5), 371-379. doi:10.1038/nri1350

Ladas, E. J., Orjuela, M., Stevenson, K., Cole, P. D., Lin, M., Athale, U. H., . . . Kelly, K. M. (2016). Dietary intake and childhood leukemia: The Diet and Acute Lymphoblastic Leukemia Treatment (DALLT) cohort study. Nutrition, 32(10), 1103-1109 e1101. doi:10.1016/j.nut.2016.03.014 Laharrague, P., Larrouy, D., Fontanilles, A. M., Truel, N., Campfield, A., Tenenbaum, R., . . Casteilla, L. (1998). High expression of leptin by human bone marrow adipocytes in primary culture. FASEB J, 12(9), 747752. doi:10.1096/fasebj.12.9.747

Lapidot, T., \& Kollet, O. (2002). The essential roles of the chemokine SDF-1 and its receptor CXCR4 in human stem cell homing and repopulation of transplanted immune-deficient NOD/SCID and NOD/SCID/B2m(null) mice. Leukemia, 16(10), 1992-2003. doi:10.1038/sj.leu.2402684 
Lee, G. H., Proenca, R., Montez, J. M., Carroll, K. M., Darvishzadeh, J. G., Lee, J. I., \& Friedman, J. M. (1996). Abnormal splicing of the leptin receptor in diabetic mice. Nature, 379(6566), 632-635. doi:10.1038/379632a0

Liang, Y., Van Zant, G., \& Szilvassy, S. J. (2005). Effects of aging on the homing and engraftment of murine hematopoietic stem and progenitor cells.

Blood, 106(4), 1479-1487. doi:10.1182/blood-2004-11-4282

Lindsley, R. C., Saber, W., Mar, B. G., Redd, R., Wang, T., Haagenson, M. D., . . . Ebert, B. L. (2017). Prognostic Mutations in Myelodysplastic Syndrome after Stem-Cell Transplantation. N Engl J Med, 376(6), 536-547. doi:10.1056/NEJMoa1611604

Lipsey, C. C., Harbuzariu, A., Daley-Brown, D., \& Gonzalez-Perez, R. R. (2016). Oncogenic role of leptin and Notch interleukin-1 leptin crosstalk outcome in cancer. World J Methodol, 6(1), 43-55. doi:10.5662/wjm.v6.i1.43

Liu, C. P., \& Auerbach, R. (1991). In vitro development of murine T cells from prethymic and preliver embryonic yolk sac hematopoietic stem cells.

Development, 113(4), 1315-1323. Retrieved from https://www.ncbi.nlm.nih.gov/pubmed/1811945

Lo Celso, C., Fleming, H. E., Wu, J. W., Zhao, C. X., Miake-Lye, S., Fujisaki, J., . . Scadden, D. T. (2009). Live-animal tracking of individual haematopoietic stem/progenitor cells in their niche. Nature, 457(7225), 9296. doi:10.1038/nature 07434 
Lord, B. I., Testa, N. G., \& Hendry, J. H. (1975). The relative spatial distributions of CFUs and CFUc in the normal mouse femur. Blood, 46(1), 65-72. Retrieved from https://www.ncbi.nlm.nih.gov/pubmed/1131427

Lord, G. M., Matarese, G., Howard, J. K., Baker, R. J., Bloom, S. R., \& Lechler, R. I. (1998). Leptin modulates the T-cell immune response and reverses starvation-induced immunosuppression. Nature, 394(6696), 897-901. doi:10.1038/29795

Lorenz, E., Uphoff, D., Reid, T. R., \& Shelton, E. (1951). Modification of irradiation injury in mice and guinea pigs by bone marrow injections. $J$ Natl Cancer Inst, 12(1), 197-201. Retrieved from https://www.ncbi.nlm.nih.gov/pubmed/14874130

Lu, R., Czechowicz, A., Seita, J., Jiang, D., \& Weissman, I. L. (2019). Clonallevel lineage commitment pathways of hematopoietic stem cells in vivo. Proc Natl Acad Sci U S A, 116(4), 1447-1456.

doi:10.1073/pnas.1801480116

Lu, Z., Xie, J., Wu, G., Shen, J., Collins, R., Chen, W., . . Zhang, C. C. (2017). Fasting selectively blocks development of acute lymphoblastic leukemia via leptin-receptor upregulation. Nat Med, 23(1), 79-90. doi: $10.1038 / \mathrm{nm} .4252$

Ma, Q., Jones, D., Borghesani, P. R., Segal, R. A., Nagasawa, T., Kishimoto, T., ... Springer, T. A. (1998). Impaired B-lymphopoiesis, myelopoiesis, and derailed cerebellar neuron migration in CXCR4- and SDF-1-deficient mice. 
Proc Natl Acad Sci U S A, 95(16), 9448-9453.

doi:10.1073/pnas.95.16.9448

Ma, X., Lim, U., Park, Y., Mayne, S. T., Wang, R., Hartge, P., . . Schatzkin, A. (2009). Obesity, lifestyle factors, and risk of myelodysplastic syndromes in a large US cohort. Am J Epidemiol, 169(12), 1492-1499.

doi:10.1093/aje/kwp074

Mantel, C. R., O'Leary, H. A., Chitteti, B. R., Huang, X., Cooper, S., Hangoc, G., . .. Broxmeyer, H. E. (2015). Enhancing Hematopoietic Stem Cell Transplantation Efficacy by Mitigating Oxygen Shock. Cell, 161(7), 15531565. doi:10.1016/j.cell.2015.04.054

Martincorena, I., \& Campbell, P. J. (2015). Somatic mutation in cancer and normal cells. Science, 349(6255), 1483-1489.

doi:10.1126/science.aab4082

Mayani, H., Wagner, J. E., \& Broxmeyer, H. E. (2020). Cord blood research, banking, and transplantation: achievements, challenges, and perspectives. Bone Marrow Transplant, 55(1), 48-61. doi:10.1038/s41409019-0546-9

McDermott, S. P., Eppert, K., Lechman, E. R., Doedens, M., \& Dick, J. E. (2010). Comparison of human cord blood engraftment between immunocompromised mouse strains. Blood, 116(2), 193-200. doi:10.1182/blood-2010-02-271841 
Medvinsky, A., Rybtsov, S., \& Taoudi, S. (2011). Embryonic origin of the adult hematopoietic system: advances and questions. Development, 138(6), 1017-1031. doi:10.1242/dev.040998

Medvinsky, A. L., Gan, O. I., Semenova, M. L., \& Samoylina, N. L. (1996).

Development of day-8 colony-forming unit-spleen hematopoietic progenitors during early murine embryogenesis: spatial and temporal mapping. Blood, 87(2), 557-566. Retrieved from https://www.ncbi.nlm.nih.gov/pubmed/8555477

Medvinsky, A. L., Samoylina, N. L., Muller, A. M., \& Dzierzak, E. A. (1993). An early pre-liver intraembryonic source of CFU-S in the developing mouse. Nature, 364(6432), 64-67. doi:10.1038/364064a0

Mendez-Ferrer, S., Lucas, D., Battista, M., \& Frenette, P. S. (2008). Haematopoietic stem cell release is regulated by circadian oscillations. Nature, 452(7186), 442-447. doi:10.1038/nature06685

Mendez-Ferrer, S., Michurina, T. V., Ferraro, F., Mazloom, A. R., Macarthur, B. D., Lira, S. A., . . Frenette, P. S. (2010). Mesenchymal and haematopoietic stem cells form a unique bone marrow niche. Nature, 466(7308), 829-834. doi:10.1038/nature09262

Miller, J. P., \& Allman, D. (2005). Linking age-related defects in B lymphopoiesis to the aging of hematopoietic stem cells. Semin Immunol, 17(5), 321-329. doi:10.1016/j.smim.2005.05.003

Min, D., Taylor, P. A., Panoskaltsis-Mortari, A., Chung, B., Danilenko, D. M., Farrell, C., . . W Weinberg, K. I. (2002). Protection from thymic epithelial cell 
injury by keratinocyte growth factor: a new approach to improve thymic and peripheral T-cell reconstitution after bone marrow transplantation. Blood, 99(12), 4592-4600. doi:10.1182/blood.v99.12.4592

Miyamoto, T., Weissman, I. L., \& Akashi, K. (2000). AML1/ETO-expressing nonleukemic stem cells in acute myelogenous leukemia with 8;21 chromosomal translocation. Proc Natl Acad Sci U S A, 97(13), 7521-7526. doi:10.1073/pnas.97.13.7521

Moore, M. A., \& Metcalf, D. (1970). Ontogeny of the haemopoietic system: yolk sac origin of in vivo and in vitro colony forming cells in the developing mouse embryo. Br J Haematol, 18(3), 279-296. doi:10.1111/j.13652141.1970.tb01443.x

Morrison, S. J., \& Scadden, D. T. (2014). The bone marrow niche for haematopoietic stem cells. Nature, 505(7483), 327-334. doi:10.1038/nature12984

Morrison, S. J., Wandycz, A. M., Akashi, K., Globerson, A., \& Weissman, I. L. (1996). The aging of hematopoietic stem cells. Nat Med, 2(9), 1011-1016. doi:10.1038/nm0996-1011

Morrison, S. J., Wandycz, A. M., Hemmati, H. D., Wright, D. E., \& Weissman, I. L. (1997). Identification of a lineage of multipotent hematopoietic progenitors. Development, 124(10), 1929-1939. Retrieved from https://www.ncbi.nlm.nih.gov/pubmed/9169840 
Morrison, S. J., \& Weissman, I. L. (1994). The long-term repopulating subset of hematopoietic stem cells is deterministic and isolatable by phenotype. Immunity, 1(8), 661-673. doi:10.1016/1074-7613(94)90037-x

Moschovi, M., Trimis, G., Vounatsou, M., Katsibardi, K., Margeli, A., Damianos, A., ... Papassotiriou, I. (2010). Serial plasma concentrations of adiponectin, leptin, and resistin during therapy in children with acute lymphoblastic leukemia. J Pediatr Hematol Oncol, 32(1), e8-13. doi:10.1097/MPH.0b013e3181b8a50c

Mouzaki, A., Panagoulias, I., Dervilli, Z., Zolota, V., Spadidea, P., Rodi, M., .. . Georgakopoulos, T. (2009). Expression patterns of leptin receptor (OB-R) isoforms and direct in vitro effects of recombinant leptin on OB-R, leptin expression and cytokine secretion by human hematopoietic malignant cells. Cytokine, 48(3), 203-211. doi:10.1016/j.cyto.2009.07.006

Muller, A. M., Medvinsky, A., Strouboulis, J., Grosveld, F., \& Dzierzak, E. (1994). Development of hematopoietic stem cell activity in the mouse embryo. Immunity, 1(4), 291-301. doi:10.1016/1074-7613(94)90081-7

Murphy, F., Kroll, M. E., Pirie, K., Reeves, G., Green, J., \& Beral, V. (2013). Body size in relation to incidence of subtypes of haematological malignancy in the prospective Million Women Study. Br J Cancer, 108(11), 2390-2398. doi:10.1038/bjc.2013.159

Myers, M. G., Jr., Leibel, R. L., Seeley, R. J., \& Schwartz, M. W. (2010). Obesity and leptin resistance: distinguishing cause from effect. Trends Endocrinol Metab, 21(11), 643-651. doi:10.1016/j.tem.2010.08.002 
Nakao, T., Hino, M., Yamane, T., Nishizawa, Y., Morii, H., \& Tatsumi, N. (1998). Expression of the leptin receptor in human leukaemic blast cells. $\mathrm{Br} \mathrm{J}$ Haematol, 102(3), 740-745. doi:10.1046/j.1365-2141.1998.00843.x

Nangalia, J., Massie, C. E., Baxter, E. J., Nice, F. L., Gundem, G., Wedge, D. C., ... Green, A. R. (2013). Somatic CALR mutations in myeloproliferative neoplasms with nonmutated JAK2. N Engl J Med, 369(25), 2391-2405. doi:10.1056/NEJMoa1312542

Naveiras, O., Nardi, V., Wenzel, P. L., Hauschka, P. V., Fahey, F., \& Daley, G. Q. (2009). Bone-marrow adipocytes as negative regulators of the haematopoietic microenvironment. Nature, 460(7252), 259-263. doi:10.1038/nature08099

Naylor, C., \& Petri, W. A., Jr. (2016). Leptin Regulation of Immune Responses. Trends Mol Med, 22(2), 88-98. doi:10.1016/j.molmed.2015.12.001

Nilsson, S. K., Johnston, H. M., \& Coverdale, J. A. (2001). Spatial localization of transplanted hemopoietic stem cells: inferences for the localization of stem cell niches. Blood, 97(8), 2293-2299. doi:10.1182/blood.v97.8.2293

Nobile, M. S., Vlachou, T., Spolaor, S., Bossi, D., Cazzaniga, P., Lanfrancone, L., ... Besozzi, D. (2019). Modeling cell proliferation in human acute myeloid leukemia xenografts. Bioinformatics, 35(18), 3378-3386.

doi:10.1093/bioinformatics/btz063

North, T., Gu, T. L., Stacy, T., Wang, Q., Howard, L., Binder, M., .. Speck, N. A. (1999). Cbfa2 is required for the formation of intra-aortic hematopoietic 
clusters. Development, 126(11), 2563-2575. Retrieved from

https://www.ncbi.nlm.nih.gov/pubmed/10226014

O'Leary, H., Ou, X., \& Broxmeyer, H. E. (2013). The role of dipeptidyl peptidase 4 in hematopoiesis and transplantation. Curr Opin Hematol, 20(4), 314-319. doi:10.1097/MOH.0b013e32836125ac

Ogawa, M., Nishikawa, S., Ikuta, K., Yamamura, F., Naito, M., Takahashi, K., \& Nishikawa, S. (1988). B cell ontogeny in murine embryo studied by a culture system with the monolayer of a stromal cell clone, ST2: B cell progenitor develops first in the embryonal body rather than in the yolk sac. EMBO J, 7(5), 1337-1343. Retrieved from https://www.ncbi.nlm.nih.gov/pubmed/3261687

Oguro, H., Ding, L., \& Morrison, S. J. (2013). SLAM family markers resolve functionally distinct subpopulations of hematopoietic stem cells and multipotent progenitors. Cell Stem Cell, 13(1), 102-116.

doi:10.1016/j.stem.2013.05.014

Okada, S., Nakauchi, H., Nagayoshi, K., Nishikawa, S., Miura, Y., \& Suda, T. (1992). In vivo and in vitro stem cell function of c-kit- and Sca-1-positive murine hematopoietic cells. Blood, 80(12), 3044-3050. Retrieved from https://www.ncbi.nlm.nih.gov/pubmed/1281687

Omatsu, Y., Sugiyama, T., Kohara, H., Kondoh, G., Fujii, N., Kohno, K., \& Nagasawa, T. (2010). The essential functions of adipo-osteogenic progenitors as the hematopoietic stem and progenitor cell niche. Immunity, 33(3), 387-399. doi:10.1016/j.immuni.2010.08.017 
Ongradi, J., \& Kovesdi, V. (2010). Factors that may impact on immunosenescence: an appraisal. Immun Ageing, 7, 7. doi:10.1186/17424933-7-7

Osawa, M., Hanada, K., Hamada, H., \& Nakauchi, H. (1996). Long-term lymphohematopoietic reconstitution by a single CD34-low/negative hematopoietic stem cell. Science, 273(5272), 242-245. doi:10.1126/science.273.5272.242

Ottinger, H. D., Beelen, D. W., Scheulen, B., Schaefer, U. W., \& Grosse-Wilde, H. (1996). Improved immune reconstitution after allotransplantation of peripheral blood stem cells instead of bone marrow. Blood, 88(7), 27752779. Retrieved from https://www.ncbi.nlm.nih.gov/pubmed/8839875

Ozturk, K., Avcu, F., \& Ural, A. U. (2012). Aberrant expressions of leptin and adiponectin receptor isoforms in chronic myeloid leukemia patients. Cytokine, 57(1), 61-67. doi:10.1016/j.cyto.2011.10.004

Papaemmanuil, E., Gerstung, M., Malcovati, L., Tauro, S., Gundem, G., Van Loo, P., . . . Chronic Myeloid Disorders Working Group of the International Cancer Genome, C. (2013). Clinical and biological implications of driver mutations in myelodysplastic syndromes. Blood, 122(22), 3616-3627; quiz 3699. doi:10.1182/blood-2013-08-518886

Park, H. Y., Kwon, H. M., Lim, H. J., Hong, B. K., Lee, J. Y., Park, B. E., . . Kim, H. S. (2001). Potential role of leptin in angiogenesis: leptin induces endothelial cell proliferation and expression of matrix metalloproteinases 
in vivo and in vitro. Exp Mol Med, 33(2), 95-102.

doi:10.1038/emm.2001.17

Pellin, D., Loperfido, M., Baricordi, C., Wolock, S. L., Montepeloso, A., Weinberg, O. K., . . Biasco, L. (2019). A comprehensive single cell transcriptional landscape of human hematopoietic progenitors. Nat Commun, 10(1), 2395. doi:10.1038/s41467-019-10291-0

Perales, M. A., Goldberg, J. D., Yuan, J., Koehne, G., Lechner, L., Papadopoulos, E. B., . . van den Brink, M. R. (2012). Recombinant human interleukin-7 (CYT107) promotes T-cell recovery after allogeneic stem cell transplantation. Blood, 120(24), 4882-4891. doi:10.1182/blood2012-06-437236

Perruccio, K., Bonifazi, P., Topini, F., Tosti, A., Bozza, S., Aloisi, T., . . Velardi, A. (2010). Thymosin alpha1 to harness immunity to pathogens after haploidentical hematopoietic transplantation. Ann N Y Acad Sci, 1194, 153-161. doi:10.1111/j.1749-6632.2010.05486.x

Pietras, E. M., Lakshminarasimhan, R., Techner, J. M., Fong, S., Flach, J., Binnewies, M., \& Passegue, E. (2014). Re-entry into quiescence protects hematopoietic stem cells from the killing effect of chronic exposure to type I interferons. J Exp Med, 211(2), 245-262. doi:10.1084/jem.20131043

Pietras, E. M., Reynaud, D., Kang, Y. A., Carlin, D., Calero-Nieto, F. J., Leavitt, A. D., . . Passegue, E. (2015). Functionally Distinct Subsets of LineageBiased Multipotent Progenitors Control Blood Production in Normal and 
Regenerative Conditions. Cell Stem Cell, 17(1), 35-46.

doi:10.1016/j.stem.2015.05.003

Pinho, S., Marchand, T., Yang, E., Wei, Q., Nerlov, C., \& Frenette, P. S. (2018). Lineage-Biased Hematopoietic Stem Cells Are Regulated by Distinct Niches. Dev Cell, 44(5), 634-641 e634. doi:10.1016/j.devcel.2018.01.016

Poulos, M. G., Ramalingam, P., Gutkin, M. C., Llanos, P., Gilleran, K., Rabbany, S. Y., \& Butler, J. M. (2017). Endothelial transplantation rejuvenates aged hematopoietic stem cell function. J Clin Invest, 127(11), 4163-4178. doi:10.1172/JCI93940

Powles, R., Mehta, J., Kulkarni, S., Treleaven, J., Millar, B., Marsden, J., . . . Singhal, S. (2000). Allogeneic blood and bone-marrow stem-cell transplantation in haematological malignant diseases: a randomised trial. Lancet, 355(9211), 1231-1237. doi:10.1016/S0140-6736(00)02090-0

Rieger, M. A., \& Schroeder, T. (2012). Hematopoiesis. Cold Spring Harb Perspect Biol, 4(12). doi:10.1101/cshperspect.a008250

Robert-Moreno, A., Espinosa, L., de la Pompa, J. L., \& Bigas, A. (2005). RBPjkappa-dependent Notch function regulates Gata2 and is essential for the formation of intra-embryonic hematopoietic cells. Development, 132(5), 1117-1126. doi:10.1242/dev.01660

Robin, C., Ottersbach, K., Durand, C., Peeters, M., Vanes, L., Tybulewicz, V., \& Dzierzak, E. (2006). An unexpected role for IL-3 in the embryonic development of hematopoietic stem cells. Dev Cell, 11(2), 171-180. doi:10.1016/j.devcel.2006.07.002 
Rossi, D. J., Bryder, D., Zahn, J. M., Ahlenius, H., Sonu, R., Wagers, A. J., \& Weissman, I. L. (2005). Cell intrinsic alterations underlie hematopoietic stem cell aging. Proc Natl Acad Sci U S A, 102(26), 9194-9199. doi:10.1073/pnas.0503280102

Sacchetti, B., Funari, A., Michienzi, S., Di Cesare, S., Piersanti, S., Saggio, I., . . . Bianco, P. (2007). Self-renewing osteoprogenitors in bone marrow sinusoids can organize a hematopoietic microenvironment. Cell, 131(2), 324-336. doi:10.1016/j.cell.2007.08.025

Sadagurski, M., Norquay, L., Farhang, J., D'Aquino, K., Copps, K., \& White, M. F. (2010). Human IL6 enhances leptin action in mice. Diabetologia, 53(3), 525-535. doi:10.1007/s00125-009-1580-8

Sarkaria, S. M., Decker, M., \& Ding, L. (2018). Bone Marrow Micro-Environment in Normal and Deranged Hematopoiesis: Opportunities for Regenerative Medicine and Therapies. Bioessays, 40(3). doi:10.1002/bies.201700190 Sarraf, P., Frederich, R. C., Turner, E. M., Ma, G., Jaskowiak, N. T., Rivet, D. J., 3rd, .. . Alexander, H. R. (1997). Multiple cytokines and acute inflammation raise mouse leptin levels: potential role in inflammatory anorexia. J Exp Med, 185(1), 171-175. doi:10.1084/jem.185.1.171

Sasaki, T. (2015). Age-Associated Weight Gain, Leptin, and SIRT1: A Possible Role for Hypothalamic SIRT1 in the Prevention of Weight Gain and Aging through Modulation of Leptin Sensitivity. Front Endocrinol (Lausanne), 6, 109. doi:10.3389/fendo.2015.00109 
Sawamiphak, S., Kontarakis, Z., \& Stainier, D. Y. (2014). Interferon gamma signaling positively regulates hematopoietic stem cell emergence. Dev Cell, 31(5), 640-653. doi:10.1016/j.devcel.2014.11.007

Scadden, D. T. (2014). Nice neighborhood: emerging concepts of the stem cell niche. Cell, 157(1), 41-50. doi:10.1016/j.cell.2014.02.013

Schneider, J. E. (2004). Energy balance and reproduction. Physiol Behav, 81(2), 289-317. doi:10.1016/j.physbeh.2004.02.007

Schofield, R. (1978). The relationship between the spleen colony-forming cell and the haemopoietic stem cell. Blood Cells, 4(1-2), 7-25. Retrieved from https://www.ncbi.nlm.nih.gov/pubmed/747780

Seita, J., \& Weissman, I. L. (2010). Hematopoietic stem cell: self-renewal versus differentiation. Wiley Interdiscip Rev Syst Biol Med, 2(6), 640-653. doi:10.1002/wsbm. 86

Shapira, S. N., \& Christofk, H. R. (2020). Metabolic Regulation of Tissue Stem Cells. Trends Cell Biol, 30(7), 566-576. doi:10.1016/j.tcb.2020.04.004 Sharma, M., Afrin, F., Satija, N., Tripathi, R. P., \& Gangenahalli, G. U. (2011). Stromal-derived factor-1/CXCR4 signaling: indispensable role in homing and engraftment of hematopoietic stem cells in bone marrow. Stem Cells Dev, 20(6), 933-946. doi:10.1089/scd.2010.0263

Shlush, L. I., Zandi, S., Mitchell, A., Chen, W. C., Brandwein, J. M., Gupta, V., . . . Dick, J. E. (2014). Identification of pre-leukaemic haematopoietic stem cells in acute leukaemia. Nature, 506(7488), 328-333. doi:10.1038/nature13038 
Sierra-Honigmann, M. R., Nath, A. K., Murakami, C., Garcia-Cardena, G., Papapetropoulos, A., Sessa, W. C., . . Flores-Riveros, J. R. (1998). Biological action of leptin as an angiogenic factor. Science, 281(5383), 1683-1686. doi:10.1126/science.281.5383.1683

Signer, R. A., Montecino-Rodriguez, E., Witte, O. N., McLaughlin, J., \& Dorshkind, K. (2007). Age-related defects in B lymphopoiesis underlie the myeloid dominance of adult leukemia. Blood, 110(6), 1831-1839. doi:10.1182/blood-2007-01-069401

Silva, A., Anderson, A. R., \& Gatenby, R. (2011). A multiscale model of the bone marrow and hematopoiesis. Math Biosci Eng, 8(2), 643-658. doi:10.3934/mbe.2011.8.643

Siminovitch, L., McCulloch, E. A., \& Till, J. E. (1963). The Distribution of ColonyForming Cells among Spleen Colonies. J Cell Comp Physiol, 62, 327-336. doi:10.1002/jcp.1030620313

Singh, L., Brennan, T. A., Russell, E., Kim, J. H., Chen, Q., Brad Johnson, F., \& Pignolo, R. J. (2016). Aging alters bone-fat reciprocity by shifting in vivo mesenchymal precursor cell fate towards an adipogenic lineage. Bone, 85, 29-36. doi:10.1016/j.bone.2016.01.014

Smith, F. J., Campfield, L. A., Moschera, J. A., Bailon, P. S., \& Burn, P. (1998). Brain administration of OB protein (leptin) inhibits neuropeptide-Y-induced feeding in ob/ob mice. Regul Pept, 75-76, 433-439. doi:10.1016/s01670115(98)00099-8 
Spangrude, G. J., Heimfeld, S., \& Weissman, I. L. (1988). Purification and characterization of mouse hematopoietic stem cells. Science, 241(4861), 58-62. doi:10.1126/science.2898810

Starling, S. (2018). Ageing: Somatic mutations accumulate in ageing and diseased neurons. Nat Rev Neurol, 14(2), 64. doi:10.1038/nrneurol.2017.181

Steensma, D. P., Bejar, R., Jaiswal, S., Lindsley, R. C., Sekeres, M. A., Hasserjian, R. P., \& Ebert, B. L. (2015). Clonal hematopoiesis of indeterminate potential and its distinction from myelodysplastic syndromes. Blood, 126(1), 9-16. doi:10.1182/blood-2015-03-631747

Stein, S. J., \& Baldwin, A. S. (2013). Deletion of the NF-kappaB subunit p65/RelA in the hematopoietic compartment leads to defects in hematopoietic stem cell function. Blood, 121(25), 5015-5024. doi:10.1182/blood-2013-02486142

Stewart, M. H., Gutierrez-Martinez, P., Beerman, I., Garrison, B., Gallagher, E. J., LeRoith, D., \& Rossi, D. J. (2014). Growth hormone receptor signaling is dispensable for HSC function and aging. Blood, 124(20), 3076-3080. doi:10.1182/blood-2014-05-575308

Storek, J., Dawson, M. A., Storer, B., Stevens-Ayers, T., Maloney, D. G., Marr, K. A., . . Boeckh, M. (2001). Immune reconstitution after allogeneic marrow transplantation compared with blood stem cell transplantation. Blood, 97(11), 3380-3389. doi:10.1182/blood.v97.11.3380 
Sudo, K., Ema, H., Morita, Y., \& Nakauchi, H. (2000). Age-associated characteristics of murine hematopoietic stem cells. J Exp Med, 192(9), 1273-1280. doi:10.1084/jem.192.9.1273

Sun, J., Ramos, A., Chapman, B., Johnnidis, J. B., Le, L., Ho, Y. J., . . . Camargo, F. D. (2014). Clonal dynamics of native haematopoiesis. Nature, 514(7522), 322-327. doi:10.1038/nature13824

Sutherland, J. S., Spyroglou, L., Muirhead, J. L., Heng, T. S., Prieto-Hinojosa, A., Prince, H. M., . . Boyd, R. L. (2008). Enhanced immune system regeneration in humans following allogeneic or autologous hemopoietic stem cell transplantation by temporary sex steroid blockade. Clin Cancer Res, 14(4), 1138-1149. doi:10.1158/1078-0432.CCR-07-1784

Talib, S., \& Shepard, K. A. (2020). Unleashing the cure: Overcoming persistent obstacles in the translation and expanded use of hematopoietic stem cellbased therapies. Stem Cells Transl Med, 9(4), 420-426. doi:10.1002/sctm.19-0375

Tan, C. K., \& Wahli, W. (2016). A trilogy of glucocorticoid receptor actions. Proc Natl Acad Sci U S A, 113(5), 1115-1117. doi:10.1073/pnas.1524215113

Taoudi, S., Gonneau, C., Moore, K., Sheridan, J. M., Blackburn, C. C., Taylor, E., \& Medvinsky, A. (2008). Extensive hematopoietic stem cell generation in the AGM region via maturation of VE-cadherin+CD45+ pre-definitive HSCs. Cell Stem Cell, 3(1), 99-108. doi:10.1016/j.stem.2008.06.004 Tartaglia, L. A., Dembski, M., Weng, X., Deng, N., Culpepper, J., Devos, R., . . . Tepper, R. I. (1995). Identification and expression cloning of a leptin 
receptor, OB-R. Cell, 83(7), 1263-1271. doi:10.1016/0092-8674(95)90151-

5

Thiagarajan, P. S., Zheng, Q., Bhagrath, M., Mulkearns-Hubert, E. E., Myers, M. G., Lathia, J. D., \& Reizes, O. (2017). STAT3 activation by leptin receptor is essential for TNBC stem cell maintenance. Endocr Relat Cancer, 24(8), 415-426. doi:10.1530/ERC-16-0349

Thomas, E. D., Lochte, H. L., Jr., Cannon, J. H., Sahler, O. D., \& Ferrebee, J. W. (1959). Supralethal whole body irradiation and isologous marrow transplantation in man. J Clin Invest, 38, 1709-1716. doi:10.1172/JCl103949

Thornton, J. E., Cheung, C. C., Clifton, D. K., \& Steiner, R. A. (1997). Regulation of hypothalamic proopiomelanocortin mRNA by leptin in ob/ob mice. Endocrinology, 138(11), 5063-5066. doi:10.1210/endo.138.11.5651

Till, J. E. (1961). Radiation effects on the division cycle of mammalian cells in vitro. Ann N Y Acad Sci, 95, 911-919. doi:10.1111/j.17496632.1961.tb50086.x

Till, J. E., \& Mc, C. E. (1961). A direct measurement of the radiation sensitivity of normal mouse bone marrow cells. Radiat Res, 14, 213-222. Retrieved from https://www.ncbi.nlm.nih.gov/pubmed/13776896

Trinh T, R. J., Aljoufi A, Cooper S, Sinn A, Srour EF, Broxmeyer HE. (2020). Leptin receptor, a surface marker for a subset of highly engrafting longterm functional hematopoietic stem cells. Leukemia. doi:10.1038/s41375020-01079-z 
Trinh, T., Ropa, J., Aljoufi, A., Cooper, S., Sinn, A., Srour, E. F., \& Broxmeyer, H. E. (2020). Leptin receptor, a surface marker for a subset of highly engrafting long-term functional hematopoietic stem cells. Leukemia. doi:10.1038/s41375-020-01079-z

Tsiotra, P. C., Pappa, V., Koukourava, A., Economopoulos, T., Tsigos, C., \& Raptis, S. A. (2005). Expression of leptin receptors in mononuclear cells from myelodysplastic syndromes and acute myeloid leukemias. Acta Haematol, 114(2), 71-77. doi:10.1159/000086578

Umemoto, Y., Tsuji, K., Yang, F. C., Ebihara, Y., Kaneko, A., Furukawa, S., \& Nakahata, T. (1997). Leptin stimulates the proliferation of murine myelocytic and primitive hematopoietic progenitor cells. Blood, 90(9), 3438-3443. Retrieved from https://www.ncbi.nlm.nih.gov/pubmed/9345027 Visnjic, D., Kalajzic, Z., Rowe, D. W., Katavic, V., Lorenzo, J., \& Aguila, H. L. (2004). Hematopoiesis is severely altered in mice with an induced osteoblast deficiency. Blood, 103(9), 3258-3264. doi:10.1182/blood-2003$11-4011$

Vormoor, J., Lapidot, T., Pflumio, F., Risdon, G., Patterson, B., Broxmeyer, H. E., \& Dick, J. E. (1994). Immature human cord blood progenitors engraft and proliferate to high levels in severe combined immunodeficient mice. Blood, 83(9), 2489-2497. Retrieved from https://www.ncbi.nlm.nih.gov/pubmed/7513200

Wagner, J. E., Jr., Brunstein, C. G., Boitano, A. E., DeFor, T. E., McKenna, D., Sumstad, D., . . Bleul, C. C. (2016). Phase I/II Trial of StemRegenin-1 
Expanded Umbilical Cord Blood Hematopoietic Stem Cells Supports Testing as a Stand-Alone Graft. Cell Stem Cell, 18(1), 144-155. doi:10.1016/j.stem.2015.10.004

Wahlestedt, M., \& Bryder, D. (2017). The slippery slope of hematopoietic stem cell aging. Exp Hematol, 56, 1-6. doi:10.1016/j.exphem.2017.09.008

Wang, T., Fahrmann, J. F., Lee, H., Li, Y. J., Tripathi, S. C., Yue, C., . . Yu, H. (2018). JAK/STAT3-Regulated Fatty Acid beta-Oxidation Is Critical for Breast Cancer Stem Cell Self-Renewal and Chemoresistance. Cell Metab, 27(6), 1357. doi:10.1016/j.cmet.2018.04.018

Wang, Y. P., \& Lei, Q. Y. (2018). Metabolite sensing and signaling in cell metabolism. Signal Transduct Target Ther, 3, 30. doi:10.1038/s41392018-0024-7

Wasik, M., Gorska, E., Popko, K., Pawelec, K., Matysiak, M., \& Demkow, U. (2006). The GIn223Arg polymorphism of the leptin receptor gene and peripheral blood/bone marrow leptin level in leukemic children. J Physiol Pharmacol, 57 Suppl 4, 375-383. Retrieved from https://www.ncbi.nlm.nih.gov/pubmed/17072067

Weiss, B. M., Vogl, D. T., Berger, N. A., Stadtmauer, E. A., \& Lazarus, H. M. (2013). Trimming the fat: obesity and hematopoietic cell transplantation. Bone Marrow Transplant, 48(9), 1152-1160. doi:10.1038/bmt.2012.201 Welch, J. S., Ley, T. J., Link, D. C., Miller, C. A., Larson, D. E., Koboldt, D. C., . . . Wilson, R. K. (2012). The origin and evolution of mutations in acute myeloid leukemia. Cell, 150(2), 264-278. doi:10.1016/j.cell.2012.06.023 
Wex, H., Ponelis, E., Wex, T., Dressendorfer, R., Mittler, U., \& Vorwerk, P. (2002). Plasma leptin and leptin receptor expression in childhood acute lymphoblastic leukemia. Int J Hematol, 76(5), 446-452.

doi:10.1007/BF02982810

Worthington, M. L., \& Cresci, G. (2011). Immune-modulating formulas: who wins the meta-analysis race? Nutr Clin Pract, 26(6), 650-655.

doi:10.1177/0884533611425799

Xia, P., Wang, S., Du, Y., Huang, G., Satoh, T., Akira, S., \& Fan, Z. (2015).

Insulin-InsR signaling drives multipotent progenitor differentiation toward lymphoid lineages. J Exp Med, 212(13), 2305-2321.

doi:10.1084/jem.20150618

Xie, Y., Yin, T., Wiegraebe, W., He, X. C., Miller, D., Stark, D., . . Li, L. (2009).

Detection of functional haematopoietic stem cell niche using real-time imaging. Nature, 457(7225), 97-101. doi:10.1038/nature07639

Yang, F., Li, B., Yang, Y., Huang, M., Liu, X., Zhang, Y., . . Yang, L. (2019).

Leptin enhances glycolysis via OPA1-mediated mitochondrial fusion to promote mesenchymal stem cell survival. Int J Mol Med, 44(1), 301-312. doi:10.3892/ijmm.2019.4189

Yang, F., Wu, R., Jiang, Z., Chen, J., Nan, J., Su, S., . . Wang, J. (2018). Leptin increases mitochondrial OPA1 via GSK3-mediated OMA1 ubiquitination to enhance therapeutic effects of mesenchymal stem cell transplantation.

Cell Death Dis, 9(5), 556. doi:10.1038/s41419-018-0579-9 
Yang, L., Bryder, D., Adolfsson, J., Nygren, J., Mansson, R., Sigvardsson, M., \& Jacobsen, S. E. (2005). Identification of Lin(-)Sca1(+)kit(+)CD34(+)FIt3short-term hematopoietic stem cells capable of rapidly reconstituting and rescuing myeloablated transplant recipients. Blood, 105(7), 2717-2723. doi:10.1182/blood-2004-06-2159

Yilmaz, M., Kis, C., Ceylan, N. O., Okan, V., Pehlivan, M., Kucukosmanoglu, E., . . Tarakcioglu, M. (2008). Serum leptin level in acute myeloid leukemia patients. Hematology, 13(1), 21-23. doi:10.1179/102453308X315771

Yilmaz, O. H., Kiel, M. J., \& Morrison, S. J. (2006). SLAM family markers are conserved among hematopoietic stem cells from old and reconstituted mice and markedly increase their purity. Blood, 107(3), 924-930. doi:10.1182/blood-2005-05-2140

Yokomizo, T., Hasegawa, K., Ishitobi, H., Osato, M., Ema, M., Ito, Y., . . . Takahashi, S. (2008). Runx1 is involved in primitive erythropoiesis in the mouse. Blood, 111(8), 4075-4080. doi:10.1182/blood-2007-05-091637

Yokota, T., Oritani, K., Takahashi, I., Ishikawa, J., Matsuyama, A., Ouchi, N., . . . Matsuzawa, Y. (2000). Adiponectin, a new member of the family of soluble defense collagens, negatively regulates the growth of myelomonocytic progenitors and the functions of macrophages. Blood, 96(5), 1723-1732. Retrieved from https://www.ncbi.nlm.nih.gov/pubmed/10961870 Zeng, Y., He, J., Bai, Z., Li, Z., Gong, Y., Liu, C., . . Liu, B. (2019). Tracing the first hematopoietic stem cell generation in human embryo by single-cell 
RNA sequencing. Cell Res, 29(11), 881-894. doi:10.1038/s41422-0190228-6

Zhang, Y., Proenca, R., Maffei, M., Barone, M., Leopold, L., \& Friedman, J. M. (1994). Positional cloning of the mouse obese gene and its human homologue. Nature, 372(6505), 425-432. doi:10.1038/372425a0

Zhang, Y., Xue, Y., Cao, C., Huang, J., Hong, Q., Hai, T., . . Zhao, J. (2017). Thyroid hormone regulates hematopoiesis via the TR-KLF9 axis. Blood, 130(20), 2161-2170. doi:10.1182/blood-2017-05-783043

Zhao, C., Xiu, Y., Ashton, J., Xing, L., Morita, Y., Jordan, C. T., \& Boyce, B. F. (2012). Noncanonical NF-kappaB signaling regulates hematopoietic stem cell self-renewal and microenvironment interactions. Stem Cells, 30(4), 709-718. doi:10.1002/stem.1050

Zheng, Q., Banaszak, L., Fracci, S., Basali, D., Dunlap, S. M., Hursting, S. D., . . . Reizes, O. (2013). Leptin receptor maintains cancer stem-like properties in triple negative breast cancer cells. Endocr Relat Cancer, 20(6), 797808. doi:10.1530/ERC-13-0329

Zhou, B. O., Yu, H., Yue, R., Zhao, Z., Rios, J. J., Naveiras, O., \& Morrison, S. J. (2017). Bone marrow adipocytes promote the regeneration of stem cells and haematopoiesis by secreting SCF. Nat Cell Biol, 19(8), 891-903. doi:10.1038/ncb3570

Zhou, B. O., Yue, R., Murphy, M. M., Peyer, J. G., \& Morrison, S. J. (2014). Leptin-receptor-expressing mesenchymal stromal cells represent the main 
source of bone formed by adult bone marrow. Cell Stem Cell, 15(2), 154168. doi:10.1016/j.stem.2014.06.008

Zhou, Y., \& Rui, L. (2013). Leptin signaling and leptin resistance. Front Med, 7(2), 207-222. doi:10.1007/s11684-013-0263-5

Zou, Y. R., Kottmann, A. H., Kuroda, M., Taniuchi, I., \& Littman, D. R. (1998). Function of the chemokine receptor CXCR4 in haematopoiesis and in cerebellar development. Nature, 393(6685), 595-599. doi:10.1038/31269 


\section{CURRICULUM VITAE}

Thao Le Phuong Trinh

\section{Education}
06/2014 - present
Medical Scientist Training Program
Indiana University School of Medicine
$08 / 2010-05 / 2014$
Biochemistry, B.S
Nutrition science, B.S.
University of Nebraska, Lincoln, NE

\section{Honors and Achievements}

$\begin{array}{ll}\text { 01/2019 - present } & \text { T32 Program in Hematopoiesis Training Grant } \\ \text { 06/2016-12/2018 } & \text { T32 Diabetes and Obesity Training Grant } \\ 08 / 2010-05 / 2014 & \text { University Honors Program } \\ 06 / 2012-05 / 2013 & \text { Undergraduate Creative Activities and Research } \\ & \text { Experiences Program (UCARE) } \\ 2013 & \text { The Mayo Clinic Summer Undergraduate Research } \\ & \text { Fellowship } \\ 2013 & \text { The Milton E. Mohr Award } \\ 2011-2013 & \text { University High Scholars } \\ 2011-2013 & \text { Dean's List, College of Arts \& Sciences } \\ 2012 & \text { Inducted into Phi Upsilon Omicron Honor Society } \\ 2011 & \text { Inducted into National Society of Collegiate Scholars }\end{array}$


Honors Society

High School, Lincoln, NE

The Gates Millennium Scholarship

2010

The Susan Thompson Buffett Foundation Scholarship

2010

The Peter Kiewit Foundation Scholarship

\section{Research Experience}

04/2017 - present

Research in Dr. Hal Broxmeyer's Lab (PhD training)

Department of Microbiology and Immunology Indiana University School of Medicine

- Project: Characterizing LEPR-expressing hematopoietic stem cells in normal hematopoiesis and transplantation.

- Techniques: Colony assay, transplantation, ex vivo expansion and lentivirus-mediated genetic manipulation in human $\mathrm{CD} 34^{+}$cells, retrovirusmediated genetic manipulation of murine bone marrow stem and progenitor cells, shRNA cloning, SEAHORSE assay, etc. 
06/2015 and 06/2016 - 04/2017 Research in Dr. Maria Grant's Lab (Ph.D. rotation, Ph.D. candidate)

Department of Ophthalmology

Indiana University School of Medicine

- Projects:

- Characterization of acupuncturemobilized mesenchymal stem cells in treating arthritis for horses and dogs

- Investigating the impact of type 2 diabetes on bone marrow-derived hematopoietic stem cell dysfunctions

- Techniques: Immunofluorescence imaging, qRT-PCR, mesenchymal stem cell culture and expansion, acellular capillary quantification, etc.

$07 / 2015$ Research in Dr. Camella Evans-Molina (Ph.D.

rotation)

Department of Medicine Indiana University School of Medicine

- Studied aberrant calcium signaling in beta-cells in type 2 diabetes

- Techniques: qRT-PCR.

$06 / 2014$ Research in Dr. Elliot Androphy's Lab (Ph.D. rotation) 
Department of Dermatology

Indiana University School of Medicine

- Studied the functions of E2 protein in human papillomavirus replication

- Techniques: Co-IP, luciferase, virus culture, etc.

08/2011-05/2014

Research in Dr. Zempleni's Lab (8 -15 hrs/week)

Department of Nutrition Science

University of Nebraska, Lincoln, NE

- Assisted a graduate student with a project on the roles of biotin in oocyte development in fruit flies.

- Tasks performed: maintenance of fruit flies, collecting female flies, preparing fly diets with different concentration of biotin and grape leaf extract, fruit flies carboxylases profile.

- Independent project on the ENO-1's biotinylation and its potential consequence of epigenetic regulation in metastasizing cell glycolysis.

- Techniques performed: overnight biotinylation of unbiotinylated-ENO1 peptide using holocarboxylase synthetase or biotinidase, 
Western blot, recombinant DNA techniques (digestion and ligation of DNA, cell transformation, colony PCR, etc), mammalian cell culture, DNA and protein extraction and purification, real-time polymerase chain reaction, ENO1-activity assay.

06/2012-05/2014

Work in Dr. Premaraj's Lab (3-6 hrs/week)

Department of Growth and Development University of Nebraska Medical Center, College of

Dentistry

- Techniques performed: Extract DNA from bacteria collected from patients' samples, mammalian cell culture maintenance.

05/2013-08/2013

Summer Research Internship in Dr. Crawford's Lab (40 hrs/week)

Department of Cancer Biology Mayo Clinic Cancer Center

- Project: "Transactivation of EGF receptor through MMP7 in LPS-treated pancreatic acinar cells." 
- Techniques performed: Pancreatic acinar cell preparation, $\mathrm{BCA}$ protein concentration, Western Blot.

Fall 2010

\author{
Research in Dr. Grass' Lab (7 hrs/week) \\ Department of Biochemistry \\ University of Nebraska, Lincoln, NE
}

- Help maintain lab's biochemical solutions for bacterial culture

- Initiated a project on the effects of metallic copper surfaces on the survival rate of Escherichia coli and Staphylococcus

- Technique performed: bacterial cell culture, bacterial cell lyses.

\title{
Extracurricular Activities
}

10/2020 - present $\quad$ Nguyen Hien Le Scholarship Foundation (English education program for outstanding students from disadvantaged background in Vietnam)

$2017-2020 \quad$ Shadowing, Good Samaritan Free Health Clinic (once a month) 2017-2018 Mentor for the Undergraduate Mentorship Program (American Physician Scientists Association) 
04/2017

IUSM

05/2011-05/2014

Fall 2013

$08 / 2013$

$08 / 2012-05 / 2013$

07/2012-05/2013

08/2011-09/2012

$04 / 2008-08 / 2012$

Lincoln, NE

$08 / 2009-12 / 2011$

Fall 2011

hrs/week)

Fall 2010

Summer 2010

hrs/week)
Director of Faculty Outreach for Evening of the Art, Volunteer, Vietnamese Buddhist Temple (1 hr/week) Human physiology teaching assistant (1 hr/week) Clinic with a heart (on-call Vietnamese interpreter) Service Chair, Phi Upsilon Omicron Volunteer, Bryan LGH Medical Center (2hrs/week) Academic tutor, WHT Learning Community Sales clerk in Chinese Food, Hy-Vee Grocery Store, Volunteer, People's Health Center (2 hrs/week) Volunteer tutor, Education Talent Search, UNL (2 Volunteer, Lincoln International Networking Community (6 hrs/month) Volunteer, Asian Cultural and Community Center (5

\section{Publications}

\section{Articles:}

1. Trinh, T. L. P., Calzi, S. L., Shaw, L. C., Yoder, M. C., \& Grant, M. B. (2016). Promoting vascular repair in the retina: can stem/progenitor cells help?. Eye and brain, 8, 113. PMID: 28539806. PMCID: PMC5398749 
2. Yan, Y., Gao, R., Trinh, T. L., \& Grant, M. B. (2017). Immunodeficiency in pancreatic adenocarcinoma with diabetes revealed by comparative genomics. Clinical Cancer Research, 23(20), 6363-6373. PMID: 28684632 PMCID: PMC6022738

3. Duan, Y., Beli, E., Li Calzi, S., Quigley, J. L., Miller, R. C., Moldovan, L., ...Trinh, T.L.P.... \& Chalam, K. V. (2018). Loss of angiotensin-converting enzyme 2 exacerbates diabetic retinopathy by promoting bone marrow dysfunction. Stem Cells, 36(9), 1430-1440. PMID: 29761600 PMCID: PMC6410700

4. Huang, X., Trinh, T., Aljoufi, A., \& Broxmeyer, H. E. (2018). Hypoxia signaling pathway in stem cell regulation: good and evil. Current stem cell reports, 4(2), 149-157. PMID: 31275803 PMCID: PMC6605067

5. Longhini, A. L. F., Salazar, T. E., Vieira, C., Trinh, T., Duan, Y., Pay, L. M., ... \& Kim, M. (2019). Peripheral blood-derived mesenchymal stem cells demonstrate immunomodulatory potential for therapeutic use in horses. PloS one, 14(3), e0212642. PMID: 30870461. PMCID: PMC6417789.

6. Trinh, T., Ropa, J., Aljoufi, A., Cooper, S., Sinn, A., Srour, E. F., \& Broxmeyer, H. E. (2020). Leptin receptor, a surface marker for a subset of highly engrafting long-term functional hematopoietic stem cells. Leukemia, 1-12. In Press. PMID: 33159180.

7. Broxmeyer HE, Liu Y, Kapur R, Orschell CM, Aljoufi A, Ropa JP, Trinh T, Burns S, Capitano ML. Fate of Hematopoiesis During Aging. What Do We 
Really Know, and What are its implications?. Stem Cell Reviews and Reports. 2020 Nov 3:1-29. PMID: 33145673. PMCID: PMC7609374.

8. Trinh T., Broxmeyer HE. (2020). Role for Leptin and Leptin Receptors in Stem Cells During Health and Diseases. Stem Cell Rev and Rep. In Press.

9. Ropa, J., Trinh, T., Aljoufi, A., \& Broxmeyer, H. E. (2021). Consequences of coronavirus infections for primitive and mature hematopoietic cells: new insights and why it matters. Current Opinion in Hematology. In Press.

\section{Manuscripts in preparation or submitted:}

1. Chen Y., Fang S., Jiang R., He J., Jin Y., Huang X., Liu S., Capitano M., Trinh T., Teng Y., Wan J., Broxmeyer HE., Guo B. Single cell RNA-seq identifies ADGRG1 as a reliable marker of human hematopoietic stem cell upon oxidative stress. (2020)

\section{Abstracts:}

1. Thao LP. Trinh, Eleni Beli, Sergio Li Calzi, Lynn Shaw, Julia V. Busik, Goldis Malek, Daniel R. Saban, Moshe Levi, Maria B. Grant. 2016. "Role of synthetic LXR agonists in stem cell fate in the diabetic mouse." IUSM Diabetes Symposium, Indianapolis, IN.

2. Thao LP. Trinh, Eleni Beli, Sugata Hazra, Lynn Shaw, Julia V. Busik, Maria B. Grant. 2017. "Synthetic LXR Agonists Enhance Vasoreparative Function and Proliferation of Bone Marrow-Derived Stem Cells in Diabetic Mouse Models." ARVO Conference. Baltimore, MD. 
3. Thao Trinh, Scott Cooper, Arafat Aljoufi, Edward Srour, and Hal E. Broxmeyer. 2019. "Leptin Receptor as a Functional Marker for Long-term Repopulating Hematopoietic Stem Cells." ASH Conference, Orlando, FL. ASH Abstract Achievement Award Recipient.

4. Thao Trinh, Arafat Aljoufi, James Ropa, Scott Cooper, Edward F. Srour, and Hal E. Broxmeyer. 2020. "Leptin Receptor as a Marker for Functional Long-Term Repopulating Hematopoietic Stem Cells." Midwest Student Biomedical Research Forum, Omaha, NE.

5. Thao Trinh, James Ropa, Arafat Aljoufi, Scott Cooper, Edward F. Srour, and Hal E. Broxmeyer. 2020. "Leptin Receptor as a Marker for Long-term Functional Hematopoietic Stem Cells." Virtual Meeting. National MD/PhD Conference.

6. Thao Trinh, James Ropa, Arafat Aljoufi, Scott Cooper, Edward F. Srour, and Hal E. Broxmeyer. 2020. "Leptin Receptor as a Marker for a Subset of Highly Engrafting Long-term Hematopoietic Stem Cells." ASH Virtual Scientific Meeting. ASH Abstract Achievement Award Recipient.

\section{Meetings attended:}

1. Thao LP. Trinh, Eleni Beli, Sergio Li Calzi, Lynn Shaw, Julia V. Busik, Goldis Malek, Daniel R. Saban, Moshe Levi, Maria B. Grant. 2016. "Role of synthetic LXR agonists in stem cell fate in the diabetic mouse." IUSM Diabetes Symposium, Indianapolis, IN. 
2. Thao LP. Trinh, Eleni Beli, Sugata Hazra, Lynn Shaw, Julia V. Busik, Maria B. Grant. 2017. "Synthetic LXR Agonists Enhance Vasoreparative Function and Proliferation of Bone Marrow-Derived Stem Cells in Diabetic Mouse Models." ARVO Conference. Baltimore, MD.

3. Thao Trinh, Scott Cooper, Arafat Aljoufi, Edward Srour, and Hal E. Broxmeyer. 2019. “Leptin Receptor as a Functional Marker for Long-term Repopulating Hematopoietic Stem Cells." ASH Conference, Orlando, FL. ASH Abstract Achievement Award Recipient.

4. Thao Trinh, Arafat Aljoufi, James Ropa, Scott Cooper, Edward F. Srour, and Hal E. Broxmeyer. 2020. "Leptin Receptor as a Marker for Functional Long-Term Repopulating Hematopoietic Stem Cells." Midwest Student Biomedical Research Forum, Omaha, NE.

5. Thao Trinh, James Ropa, Arafat Aljoufi, Scott Cooper, Edward F. Srour, and Hal E. Broxmeyer. 2020. "Leptin Receptor As a Marker for Long-term Functional Hematopoietic Stem Cells." Virtual Meeting. National MD/PhD Conference.

6. Thao Trinh, James Ropa, Arafat Aljoufi, Scott Cooper, Edward F. Srour, and Hal E. Broxmeyer. 2020. "Leptin Receptor As a Marker for a Subset of Highly Engrafting Long-term Hematopoietic Stem Cells." ASH Virtual Scientific Meeting. ASH Abstract Achievement Award Recipient. 